\title{
WIGNER MEASURES AND OBSERVABILITY FOR THE SCHRÖDINGER EQUATION ON THE DISK
}

\author{
NALINI ANANTHARAMAN, MATTHIEU LÉAUTAUD, AND FABRICIO MACIÀ
}

\begin{abstract}
We analyse the structure of semiclassical and microlocal Wigner measures for solutions to the linear Schrödinger equation on the disk, with Dirichlet boundary conditions.

Our approach links the propagation of singularities beyond geometric optics with the completely integrable nature of the billiard in the disk. We prove a "structure theorem", expressing the restriction of the Wigner measures on each invariant torus in terms of second-microlocal measures. They are obtained by performing a finer localization in phase space around each of these tori, at the limit of the uncertainty principle, and are shown to propagate according to Heisenberg equations on the circle.

Our construction yields as corollaries (a) that the disintegration of the Wigner measures is absolutely continuous in the angular variable, which is an expression of the dispersive properties of the equation; (b) an observability inequality, saying that the $L^{2}$-norm of a solution on any open subset intersecting the boundary (resp. the $L^{2}$-norm of the Neumann trace on any nonempty open set of the boundary) controls its full $L^{2}$-norm (resp. $H^{1}$-norm). These results show in particular that the energy of solutions cannot concentrate on periodic trajectories of the billiard flow other than the boundary.
\end{abstract}

\section{CONTENTS}

1. Introduction 2

1.1. Motivation 2

1.2. Some consequences of our structure theorem 4

1.3. Stationary solutions to (1.1): eigenfunctions on the disk 5

1.4. The semiclassical viewpoint 7

$\begin{array}{lll}\text { 1.5. } & \text { The structure theorem } & 7\end{array}$

1.6. Relations to other works 8

2. The structure Theorem 10

2.1. Wigner measures: microlocal versus semiclassical point of view 10

2.2. The billiard flow 11

2.3. Standard facts about Wigner measures 13

2.4. The structure theorem: semiclassical formulation 14

2.5. The structure theorem: microlocal formulation 16

2.6. Link between microlocal and semiclassical Wigner measures 18

2.7. Application to the regularity of limit measures 19

2.8. Measures at the boundary 20

2.9. Plan of the proofs 22

3. Action-angle coordinates and decomposition of invariant measures 22

3.1. Action-angle coordinates and their quantization 22

3.2. Decomposition of an invariant measure of the billiard 24

4. Second microlocalization on a rational angle 26

4.1. Classes of test functions 26

4.2. Coordinates adapted to the second microlocalization on $\mathcal{I}_{\alpha_{0}} \quad 27$

4.3. Second microlocalization 29

4.4. Structure and propagation of $\mu^{\alpha_{0}} 32$

Key words and phrases. Semiclassical measures, microlocal defect measures, Schrödinger equation, disk, observability, completely integrable dynamics. 
4.5. Second microlocal structure of $\mu_{\alpha_{0}} \quad 33$

4.6. Propagation law for $\rho_{\alpha_{0}} \quad 36$

5. End of the semiclassical construction: proof of Theorem 2.4 40

5.1. A proof of Proposition 2.8 40

5.2. End of the proof of Theorem 2.4 40

6. The microlocal construction: sketch of the proof of Theorem 2.6

6.1. Structure of $\mu_{c} \quad 41$

6.2. Structure of $\mu^{\infty}$ and proof of Theorem 2.6

7. Proof of Theorems 1.2 and 1.3: Observability inequalities 43

7.1. Unique continuation for microlocal measures 43

7.2. Interior observability inequality: proof of Theorem $1.2 \quad 45$

7.3. Boundary observability inequality: proof of Theorem 1.3 47

Appendix A. From action-angle coordinates to polar coordinates 49

Appendix B. Commutators $\quad 54$

B.1. Formal calculation of $\left[\Delta, \mathcal{A}_{E}(P)\right] \quad 55$

B.2. Identification $\quad 56$

B.3. Formal calculation of $\left[\Delta, \mathcal{A}_{H}(P)\right] \quad 57$

Appendix C. Regularity of boundary data and consequences $\quad 58$

Appendix D. Time regularity of Wigner measures $\quad 62$

References $\quad 63$

\section{Introduction}

1.1. Motivation. We consider the unit disk

$$
\mathbb{D}=\left\{z=(x, y) \in \mathbb{R}^{2},|z|^{2}=x^{2}+y^{2}<1\right\} \subset \mathbb{R}^{2}
$$

and denote by $\Delta$ the euclidean Laplacian. We are interested in understanding dynamical properties of the (time-dependent) linear Schrödinger equation

$$
\begin{gathered}
\frac{1}{i} \frac{\partial u}{\partial t}(z, t)=\left(-\frac{1}{2} \Delta+V(t, z)\right) u(z, t), \quad t \in \mathbb{R}, \quad z=(x, y) \in \mathbb{D} \\
u\rceil_{t=0}=u^{0} \in L^{2}(\mathbb{D})
\end{gathered}
$$

with Dirichlet boundary condition $u\rceil_{\partial \mathbb{D}}=0$ (we shall write $\Delta=\Delta_{D}$ when we want to stress that we are using the Laplacian with that boundary condition). We assume that $V$ is a smooth real-valued potential, say $V \in C^{\infty}(\mathbb{R} \times \overline{\mathbb{D}} ; \mathbb{R})$. We shall denote by $U_{V}(t)$ the (unitary) propagator starting at time 0 , such that $u(\cdot, t)=U_{V}(t) u^{0}$ is the unique solution of (1.1)-(1.2).

This equation is aimed at describing the evolution of a quantum particle trapped in a diskshaped cavity, $u(\cdot, t)$ being the wave-function at time $t$. The total $L^{2}$-mass of the solution is preserved: $\|u(\cdot, t)\|_{L^{2}(\mathbb{D})}=\left\|u^{0}\right\|_{L^{2}(\mathbb{D})}$ for all time $t \in \mathbb{R}$. Thus, if the initial datum is normalized, $\left\|u^{0}\right\|_{L^{2}(\mathbb{D})}=1$, the quantity $|u(z, t)|^{2} d z$ is, for every fixed $t$, a probability density on $\mathbb{D}$; given $\Omega \subset \mathbb{D}$, the expression:

$$
\int_{\Omega}|u(z, t)|^{2} d z
$$

is the probability of finding the particle in the set $\Omega$ at time $t$. Having $\int_{0}^{T} \int_{\Omega}|u(z, t)|^{2} d x d t \geq c_{0}>0$ for all solutions of (1.1) means that every quantum particle spends a positive fraction of time of the interval $(0, T)$ in the set $\Omega$. A major issue in mathematical quantum mechanics is to describe the possible localization - or delocalization - properties of solutions to the Schrödinger equation (1.1), by which we mean the description of the distribution of the probability densities $|u(z, t)|^{2} d z$ for all solutions $u$. A more tractable problem consists in considering instead of single, fixed solutions, sequences $\left(u_{n}\right)_{n \in \mathbb{N}}$ of solutions to (1.1) and describe the asymptotic properties of the associated probability densities $\left|u_{n}(z, t)\right|^{2} d z$ or $\left|u_{n}(z, t)\right|^{2} d z d t$. This point of view still allows to deduce properties of single solutions $u$ and their distributions $|u(z, t)|^{2} d z$, as we shall see in the sequel. 
It is always possible to extract a subsequence that converges weakly:

$$
\int_{\mathbb{D} \times \mathbb{R}} \phi(z, t)\left|u_{n}(z, t)\right|^{2} d z d t \longrightarrow \int_{\overline{\mathbb{D}} \times \mathbb{R}} \phi(z, t) \nu(d z, d t), \quad \text { for every } \phi \in C_{c}(\overline{\mathbb{D}} \times \mathbb{R}),
$$

where $\nu$ is a nonnegative Radon measure on $\overline{\mathbb{D}} \times \mathbb{R}$ that describes the asymptotic mass distribution of the sequence of solutions $\left(u_{n}\right)$. One of the goals of this paper is to understand how the fact that $\left(u_{n}\right)$ solves (1.1) influences the structure of the associated measure $\nu$.

As an application, we aim at understanding the observability problem for the Schrödinger equation: given an open set $\Omega \subset \mathbb{D}$ and a time $T>0$, does there exist a constant $C=C(\Omega, T)>0$ such that we have:

$$
\int_{0}^{T} \int_{\Omega}|u(z, t)|^{2} d z d t \geq C\left\|u^{0}\right\|_{L^{2}(\mathbb{D})}^{2}, \text { for all } u^{0} \in L^{2}(\mathbb{D}) \text { and } u \text { associated solution of (1.1)? }
$$

If such an estimate holds, then every quantum particle must leave a trace on the set $\Omega$ during the time interval $(0, T)$; in other words: it is observable from $\Omega \times(0, T)$. This question is linked to that of understanding the structure of the limiting measures $\nu$. Estimate (1.3) is not satisfied if and only if there exists a sequence of data $\left(u_{n}^{0}\right)$ such that $\left\|u_{n}^{0}\right\|_{L^{2}(\mathbb{D})}=1$ and $\int_{0}^{T} \int_{\Omega}\left|u_{n}(z, t)\right|^{2} d z d t \rightarrow 0$, where $u_{n}$ is the solution of (1.1) issued from $u_{n}^{0}$. After the extraction of a subsequence, this holds if and only if the associated limit measure $\nu$ satisfies

$$
\int_{0}^{T} \int_{\overline{\mathbb{D}}} \nu(d z, d t)=T, \quad \int_{0}^{T} \int_{\Omega} \nu(d z, d t)=0 .
$$

The question of observability from $\Omega \times(0, T)$ may hence be reformulated as: can sequences of solution of (1.1) concentrate on sets which do not intersect $\Omega \times(0, T)$ ? From the point of view of applications, it is of primary interest to understand which sets $\Omega$ do observe all quantum particles trapped in a disk. Moreover, the observability of (1.1) is equivalent to the controllability of the Schrödinger equation (see e.g. [Leb92]), which means that it is possible to drive any initial condition to any final condition at time $T$, with a control (a forcing term in the right-hand side of (1.1)) located within $\Omega$.

It is well-known that the space of position variables $(z, t)$ does not suffice to describe the propagation properties of solutions to Schrödinger equations (or more generally wave equations) in the high frequency régime. To take the latter into account, one has to add the associated dual variables, $(\xi, H) \in \mathbb{R}^{2} \times \mathbb{R}$ (momentum and energy) and lift the measure $\nu$ to the phase space, associated to the variables $(z, t, \xi, H)$ : this gives rise to the so-called Wigner measures [Wig32]. We shall hence investigate the regularity and localization properties in position and momentum variables of the Wigner measures associated with sequences of normalized solutions of (1.1). They describe how the solutions are distributed over phase space. We shall develop both the microlocal and semiclassical points of view. These are two slightly different, but closely related, approaches to the problem : the semiclassical approach is more suitable when our initial data possess a welldefined oscillation rate, whereas the microlocal approach describes the singularities of solutions, independently of the choice of a scale of oscillation, at the price of giving slightly less precise results.

Our study fits in the regime of the "quantum-classical correspondence principle" , which asserts that the high-frequency dynamics of the solutions to (1.1) are described in terms of the corresponding classical dynamics; in our context the underlying classical system is the billiard flow on D. Wigner measures carry this information, for they are known to be invariant by this flow.

Of course, one may consider similar questions for any bounded domain of $\mathbb{R}^{d}$ or any Riemannian manifold, and not only the disk $\mathbb{D}$. As a matter of fact, the answer to these questions depends strongly on the dynamics of the billiard flow (resp. the geodesic flow on a Riemannian manifold), and, to our knowledge, it is known only in few cases (see Section 1.6). For instance, on negatively curved manifolds, the celebrated Quantum Unique Ergodicity conjecture remains to this day open. Two geometries for which the observation problem is well-understood, and the Wigner measures are rather well-described, are the torus $\mathbb{T}^{d}$ (see [Jaf90, Kom92, Mac11, BZ12] and [Jak97, Bou97, Mac10, AM14]) and the sphere $\mathbb{S}^{d}$, or more generally, manifolds all of whose geodesics are closed 
(see [JZ99, Mac08, Mac09, AM10]), on which the classical dynamics is completely integrable. We shall later on compare these two situations with our results on the disk $\mathbb{D}$. We refer to the article [AM12] for a survey of recent results concerning Wigner measures associated to sequences of solutions to the time-dependent Schrödinger equation in various geometries and to the review article [Lau14] on the observability question.

1.2. Some consequences of our structure theorem. Our central results are Theorems 2.4 and 2.6 below, which provide a detailed structure of the Wigner measures associated to sequences of solutions to the Schrödinger equation, using notions of second-microlocal calculus. As corollaries of these structure Theorems, we obtain:

- Corollary 1.1 (see also Theorem 2.9), which reflects the dispersive character of the Schrödinger equation (1.1);

- Theorem 1.2 (resp. Theorem 1.3), which states the observability/controllability of the equation from any nonempty open set touching the boundary of the disk (resp. from any nonempty open set of the boundary).

Let us first state these corollaries in order to motivate the more technical results of this paper.

Corollary 1.1. Let $\left(u_{n}^{0}\right)$ be a sequence in $L^{2}(\mathbb{D})$, such that $\left\|u_{n}^{0}\right\|_{L^{2}(\mathbb{D})}=1$ for all $n$. Consider the sequence of nonnegative Radon measures $\nu_{n}$ on $\overline{\mathbb{D}} \times \mathbb{R}$, defined by

$$
\nu_{n}(d z, d t)=\left|U_{V}(t) u_{n}^{0}(z)\right|^{2} d z d t .
$$

Let $\nu$ be any weak-* limit of the sequence $\left(\nu_{n}\right)$ : then $\nu(d z, d t)=\nu_{t}(d z) d t$ where, for almost every $t, \nu_{t}$ is a probability measure on $\overline{\mathbb{D}}$, and $\left.\nu_{t}\right\rceil_{\mathbb{D}}$ is absolutely continuous.

This result shows that the weak-* accumulation points of the densities (1.4) possess some regularity in the interior of the disk. This result cannot be extended to $\overline{\mathbb{D}}$, since it is easy to exhibit sequences of solutions that concentrate singularly on the boundary (the so-called whispering-gallery modes, see Section 1.3). In Theorem 2.9 below, we present a stronger version of Corollary 1.1 describing (in phase space) the regularity of microlocal lifts of such limit measures $\nu$. This precise description (as well as all results of this paper) relies on the complete integrability of the billiard flow on the disk. Its statement needs the introduction of action angle coordinates and associated invariant tori, and is postponed to Section 2.7.

The second class of results mentioned above is related to unique continuation-type properties of the Schrödinger equation (1.1). We consider the following condition on an open set $\Omega \subset \overline{\mathbb{D}}$, a time $T>0$ and a potential $V$ :

$\left.\left(\mathrm{UCP}_{V, \Omega, T}\right) \quad\left(u^{0} \in L^{2}(\mathbb{D}), \quad U_{V}(t) u^{0}\right\rceil_{(0, T) \times \Omega}=0\right) \quad \Longrightarrow \quad u^{0}=0$.

As a consequence of Theorem 2.6, we shall also prove the following quantitative version of $\left(\mathrm{UCP}_{V, \Omega, T}\right)$.

Theorem 1.2. Let $\Omega \subset \overline{\mathbb{D}}$ be an open set such that $\Omega \cap \partial \mathbb{D} \neq \emptyset$ and $T>0$. Assume one of the following statements holds:

- the potential $V \in C^{\infty}([0, T] \times \overline{\mathbb{D}} ; \mathbb{R})$, the time $T$, and the open set $\Omega$ satisfy $\left(\mathrm{UCP}_{V, \Omega, T}\right)$,

- the potential $V \in C^{\infty}(\overline{\mathbb{D}} ; \mathbb{R})$ does not depend on $t$.

Then there exists $C=C(V, \Omega, T)>0$ such that:

$$
\left\|u^{0}\right\|_{L^{2}(\mathbb{D})}^{2} \leq C \int_{0}^{T}\left\|U_{V}(t) u^{0}\right\|_{L^{2}(\Omega)}^{2} d t,
$$

for every initial datum $u^{0} \in L^{2}(\mathbb{D})$.

Roughly speaking, this means that any set $\Omega$ touching $\partial \mathbb{D}$ observes all quantum particles trapped in the disk. As we shall see, these are the only sets satisfying this property (see Section 1.3 and Remark 2.11).

We are also interested in the boundary analogue of $\left(\mathrm{UCP}_{V, \Omega, T}\right)$ for a given potential $V$, a time $T>0$ and an open set $\Gamma \subset \partial \mathbb{D}$ :

$$
\left.\left(\mathrm{UCP}_{V, \Gamma, T}\right) \quad\left(u^{0} \in H_{0}^{1}(\mathbb{D}), \quad \partial_{n}\left(U_{V}(t) u^{0}\right)\right\rceil_{(0, T) \times \Gamma}=0\right) \quad \Longrightarrow \quad u^{0}=0,
$$


where $\partial_{n}=\frac{\partial}{\partial n}$ denotes the exterior normal derivative to $\partial \mathbb{D}$. As a consequence of Theorem 2.6, we shall also prove the following quantitative version of $\left(\mathrm{UCP}_{V, \Gamma, T}\right)$.

Theorem 1.3. Let $\Gamma$ be any nonempty subset of $\partial \mathbb{D}$ and $T>0$. Suppose one of the following holds:

- the potential $V \in C^{\infty}([0, T] \times \overline{\mathbb{D}})$, the time $T$ and the set $\Gamma$ satisfy $\left(\mathrm{UCP}_{V, \Gamma, T}\right)$,

- $V \in C^{\infty}(\overline{\mathbb{D}})$ does not depend on $t$.

Then there exists $C=C(V, \Gamma, T)>0$ such that:

$$
\left\|u^{0}\right\|_{H^{1}(\mathbb{D})}^{2} \leq C \int_{0}^{T}\left\|\partial_{n}\left(U_{V}(t) u^{0}\right)\right\|_{L^{2}(\Gamma)}^{2} d t
$$

for every initial datum $u^{0} \in H_{0}^{1}(\mathbb{D})$.

Note that the unique continuation properties $\left(\mathrm{UCP}_{V, \Omega, T}\right)$ and $\left(\mathrm{UCP}_{V, \Gamma, T}\right)$ are known to hold, for instance, when $V$ is analytic in $(t, z)$, as a consequence of the Holmgren uniqueness theorem as stated by Hörmander (see e.g. [Hör76, Theorem 5.3.1]).

These three results express a delocalization property of the energy of solutions to (1.1). The observation of the $L^{2}$-norm restricted to any open set of the disk touching the boundary is sufficient to recover linearly the norm of the data. In particular, the $L^{2}$-mass of solutions cannot concentrate on periodic trajectories of the billiard. The observability inequalities (1.5) and (1.6) are especially relevant in control theory (see [Lio88, BLR92, Leb92]): in turn, they imply a controllability result from the set $\Omega$ or $\Gamma$.

As a consequence of the observability inequality 1.5, we have the following result (where we use the notation of Corollary 1.1).

Corollary 1.4. For every open set $\Omega \subset \overline{\mathbb{D}}$ touching the boundary, for every $T>0$, there exists a constant $C(T, \Omega)>0$ such that for any initial data $\left(u_{n}^{0}\right)$ and any weak-* limit $\nu$ of the sequence $\left(\nu_{n}\right)$ as in Corollary 1.1, we have

$$
\int_{0}^{T} \nu_{t}(\Omega) d t \geq \frac{1}{C(T, \Omega)}
$$

This translates the fact that any solution has to leave positive mass on any set $\Omega$ touching the boundary $\partial \mathbb{D}$ during the time interval $(0, T)$. This may be rephrased by saying that any such set observes all quantum particles trapped in the disk.

1.3. Stationary solutions to (1.1): eigenfunctions on the disk. If the potential $V(t, z)$ does not depend on the time variable $t$, we have as particular solutions of the Schrödinger equation the "stationary solutions", those with initial data given by eigenfunctions of the elliptic operator $-\Delta_{D}+2 V(z)$ involved.

In the absence of potential, i.e. if $V=0$, these solutions are well understood: the eigenfunctions of $-\Delta_{D}$ on $\mathbb{D}$ are the functions whose (non-normalized) expression in polar coordinates $(x=$ $-r \sin u, y=r \cos u)$ is

$$
\psi_{n, k}^{ \pm}\left(r e^{i u}\right)=J_{n}\left(\alpha_{n, k} r\right) e^{ \pm i n u},
$$

where $n, k$ are non-negative integers, $J_{n}$ is the $n$-th Bessel function, and the $\alpha_{n, k}$ are its positive zeros ordered increasingly with respect to $k$. The corresponding eigenvalue is $\alpha_{n, k}^{2}$. Putting then $u^{0}=\psi_{n, k}^{ \pm}$gives a time-periodic solution $u(\cdot, t)=e^{-i t \frac{\alpha_{n, k}^{2}}{2}} \psi_{n, k}^{ \pm}$to (1.1)-(1.2). Moreover, the eigenvalues of $-\Delta_{D}$ have multiplicity two. This is a consequence of a celebrated result by Siegel [Sie29], showing that $J_{n}, J_{m}$ have no common zeroes for $n \neq m$. In particular, the limit measures associated to sequences of eigenfunctions are explicitly computable in terms of the limits of the stationary distributions:

$$
\frac{\left|\psi_{n, k}^{ \pm}(z)\right|^{2}}{\left\|\psi_{n, k}^{ \pm}\right\|_{L^{2}(\mathbb{D})}^{2}} d z=\frac{\left|J_{n}\left(\alpha_{n, k} r\right)\right|^{2}}{\left\|\psi_{n, k}^{ \pm}\right\|_{L^{2}(\mathbb{D})}^{2}} r d r d u
$$


as the frequency $\alpha_{n, k}$ tends to infinity (this expression has to be slightly modified when considering linear combinations of the two eigenfunctions $\psi_{n, k}^{+}$and $\psi_{n, k}^{-}$, corresponding to the same eigenvalue, with $n$ fixed and $k$ tending to infinity) . Let us recall some particular cases of this construction. For fixed $k$ and for $n \rightarrow \infty$, it is classical [Lag83, Lemma 3.1] that

$$
\frac{\left|\psi_{n, k}^{ \pm}(z)\right|^{2}}{\left\|\psi_{n, k}^{ \pm}\right\|_{L^{2}(\mathbb{D})}^{2}} d z \rightarrow(2 \pi)^{-1} \delta_{\partial \mathbb{D}}
$$

which corresponds to the so-called whispering gallery modes. On the other hand, letting $k, n \rightarrow \infty$ with $n / k$ being constant, one may obtain for any $\gamma \in[0,1)$ depending on the ratio $n / k$ [PTZ14, Section 4.1]

$$
\frac{\left|\psi_{n, k}^{ \pm}(z)\right|^{2}}{\left\|\psi_{n, k}^{ \pm}\right\|_{L^{2}(\mathbb{D})}^{2}} d z \rightarrow \frac{1}{2 \pi\left(1-\gamma^{2}\right)^{1 / 2}} \frac{1}{\left(|z|^{2}-\gamma^{2}\right)^{1 / 2}} \mathbb{1}_{(\gamma, 1)}(|z|) d z .
$$

Except the Dirac measure on the boundary, these measures all belong to $L^{p}(\mathbb{D})$ for any $p<2$ (hence satisfying Corollary 1.1) and are invariant by rotation and positive on the boundary (hence satisfying Corollary 1.4). These measures in fact enjoy more regularity and symmetry than those asserted by Corollaries 1.1 and 1.4.

The observability question for eigenfunctions can also be simply handled in account of the bounded multiplicity of the spectrum. For any non-empty open set $\Omega_{I_{1}, I_{2}}=\left\{r e^{i u}, r \in I_{1}, u \in\right.$ $\left.I_{2}\right\} \subset \mathbb{D}$ (where $I_{1}$ is an open subset of $[0,1], I_{2}$ an open interval of $\mathbb{S}^{1}$ ), for any eigenfunction $\psi$ of $-\Delta_{D}$, one has:

$$
\|\psi\|_{L^{2}\left(\Omega_{I_{1}, \mathbb{S}^{1}}\right)} \leq C\left(\left|I_{2}\right|\right)\|\psi\|_{L^{2}\left(\Omega_{I_{1}, I_{2}}\right)}
$$

where $C\left(\left|I_{2}\right|\right)$ is a positive constant depending only on the size of $I_{2}$. On the other hand, if $\Omega_{I_{1}, I_{2}}$ touches the boundary $\left(1 \in I_{1}\right)$ it automatically satisfies the geometric control condition as defined in [BLR92, Leb92]. The results on those references imply that:

$$
\|\psi\|_{L^{2}(\mathbb{D})} \leq C^{\prime}\left(I_{1}\right)\|\psi\|_{L^{2}\left(\Omega_{I_{1}, \mathbb{S}^{1}}\right)} .
$$

Therefore, for such $\Omega_{I_{1}, I_{2}}$, we have

$$
\|\psi\|_{L^{2}(\mathbb{D})} \leq C\left(\left|I_{2}\right|\right) C^{\prime}\left(I_{1}\right)\|\psi\|_{L^{2}\left(\Omega_{I_{1}, I_{2}}\right)} .
$$

It is not known to the authors whether or not any of the results of the present article could be deduced directly from the result for eigenfunctions, even when the potential vanishes identically. This does not seem to appear in the literature. On flat tori, proving observability or regularity of Wigner measures associated to the Schrödinger equation from the explicit expression of the solutions in terms of Fourier series requires a careful analysis of the distribution of lattice points on paraboloids [Jak97, Bou97] or sophisticated arguments on lacunary Fourier series [Jaf90, Kom92]. On the disk, and in absence of a potential, one could try to expand the kernel of $e^{i t \Delta_{D} / 2}$ in terms of Bessel functions:

$$
e^{i t \Delta_{D} / 2}=\sum_{n, k, \pm} e^{-i t \alpha_{n, k}^{2} / 2}\left|\psi_{n, k}^{ \pm}\right\rangle\left\langle\psi_{n, k}^{ \pm}\right|
$$

and to use some of their known properties. Such an approach would anyway require some very technical work on the spacings between the $\alpha_{n, k}$.

Here, instead, we establish directly the links between the completely integrable nature of the dynamics of the billiard flow and the delocalization and dispersion properties of the solutions to the Schrödinger equation. Note that all results of this paper also hold for eigenfunctions of the operator $-\Delta_{D}+2 V(z)$ (as stationary solutions to (1.1)). As a matter of fact, our approach is more general for it applies as well to quasimodes and clusters of eigenfunctions of the operator $-\Delta_{D}+2 V(z)$. The reader is referred to [ALM15] and Remark 2.5 for more details on this matter. 
1.4. The semiclassical viewpoint. In spite of the fact that our statements and proofs are formulated exclusively in terms of the non-semiclassical Schrödinger equation (1.1), our results do have an interpretation in the light of the semiclassical limit for the Schödinger equation. Suppose that $v_{h}$ solves the semiclassical Schrödinger equation:

$$
\left.\frac{h}{i} \frac{\partial v_{h}}{\partial t}(z, t)=\left(-\frac{h^{2}}{2} \Delta+h^{2} V(h t, z)\right) v_{h}(z, t), \quad v_{h}\right\rceil_{t=0}=u^{0} .
$$

It turns out that $u(\cdot, t):=v_{h}(\cdot, t / h)$ is in fact the solution to the (non-semiclassical) Schrödinger equation (1.1) with initial datum $u^{0}$. As a consequence, describing properties of solutions to (1.1) on time intervals of size of order 1 amounts to describing properties of solutions to the semiclassical Schrödinger equation (1.8) up to times of order $1 / h$. Our results show that the semiclassical approximation (meaning that the solution to (1.8) should be well-approximated by its initial datum propagated through the billiard flow) breaks down in time $1 / h$. For instance, if we take as initial datum in (1.8) a coherent state localized at $\left(z_{0}, \xi_{0}\right) \in \mathbb{D} \times \mathbb{R}^{2}$,

$$
u_{n}^{0}=\frac{1}{h_{n}^{\alpha}} \rho\left(\frac{z-z_{0}}{h_{n}^{\alpha}}\right) e^{\frac{i}{h_{n}} z \cdot \xi_{0}}, \quad \rho \in C_{c}^{\infty}(\mathbb{D}), \quad \rho(0)=1, \quad \alpha \in(0,1), \quad h_{n} \rightarrow 0,
$$

our results imply that the associated solution of (1.8) on the time interval $\left(0,1 / h_{n}\right)$ is no longer concentrated on the billiard trajectory issued from $\left(z_{0}, \xi_{0}\right)$. Instead, we show that it spreads on the disk $\mathbb{D}$ (the associated measure is absolutely continuous) and it leaves a positive mass on any set touching the boundary (even if the trajectory of the billiard issued from $\left(z_{0}, \xi_{0}\right)$ avoids this set).

Hence, our analysis goes far beyond the well-understood semiclassical limit for times of order 1 , or even of order $\log (1 / h)$ (known as the Ehrenfest time, see [BR02]). Such a long time analysis is possible thanks to the complete integrability of the system. In fact, in the paper [AFKM14], which deals with the Schrödinger equation (and more general completely integrable systems) on the flat torus, it is shown that the time scale $1 / h$ is exactly the one at which the delocalization of solutions takes place; for chaotic systems on the contrary, the semiclassical approximation is expected to break down at the Ehrenfest time [Ana08, AR12, AM12].

1.5. The structure theorem. We would like to stress the fact that all these results are obtained as consequences of our main theorem, Theorem 2.4 or its variant Theorem 2.6, that gives a precise description of the structure of Wigner measures arising from solutions to (1.1). It provides a unified framework from which to derive simultaneously the absolute continuity of projections of semiclassical measures (a fact that is related to dispersive effects) on the one hand, and, on the other hand, the observability estimates (1.5) and (1.6), which are quantitative unique continuationtype properties. Since a precise statement requires the introduction of many other objects, we postpone it to Sections 2.4 and 2.5 (semiclassical and microlocal formulations respectively), and only give a rough idea of the method for the moment.

The standard construction of the Wigner measures, outlined in Section 2.5, allows to lift a measure $\nu$ to a measure $\mu^{\infty}$ on phase space (or $\mu_{s c}$ in the semiclassical setting): this is the associated microlocal defect measure [Gér91b]. The law of propagation of singularities for equation (1.1) implies that $\mu^{\infty}$ is invariant by the billiard flow in the disk, and we want to exploit the complete integrability of this flow.

For this, we use action-angle coordinates to integrate the dynamics of the billiard flow and describe associated invariant tori (Section 3.1). The angular momentum $J$ of a point $(z, \xi)$ in phase space is preserved by the flow, and so is the Hamiltonian $E=|\xi|$. The actions $J$ and $E$ are in involution and independent, except at the points of $\partial \mathbb{D}$ with tangent momentum. The angle $\alpha$ that a trajectory makes when bouncing on the boundary is a also preserved quantity (in fact a function of $J / E$ ). The key point of our proof is to analyze in detail the possible concentration of sequences on the sets $\mathcal{I}_{\alpha_{0}}=\left\{\alpha=\alpha_{0}\right\}$ of all points of phase space sharing a common incidence/reflection angle $\alpha_{0}$. To this aim, we perform a second microlocalization on this set, in the spirit of [Mac10, AM14, AFKM14]. We decompose a Wigner measure as a sum of measures supported on these invariant sets. The case $\alpha_{0} \notin \pi \mathbb{Q}$ corresponds to trajectories hitting the boundary in a dense set, and is trivial for us since it supports only one invariant 
measure. We focus on those $\mathcal{I}_{\alpha_{0}}$ for which $\alpha_{0} \in \pi \mathbb{Q}$. Any trajectory of the billiard having this angle is periodic. We wish to "zoom" on this torus to describe the concentration of the associated measure. Assuming that the initial sequence has a typical oscillation scale of order $1 / h$, we perform a second microlocalization at scale 1, which is the limit of the Heisenberg uncertainty principle. Roughly speaking, the idea is to relocalize in the action variable $J$ at scale 1 (i.e. $h$ times $1 / h)$, so that the Heisenberg uncertainty principle implies delocalization in the conjugated angle variable. We obtain two limit objects, interpreted as second-microlocal measures. The first one captures the part of our sequence of solutions whose derivatives in directions "transverse to the flow" remain bounded; the second one captures the part of the solution rapidly oscillating in these directions. Understanding the notion of transversality adapted to this problem is achieved by constructing a flow that interpolates between the billiard flow (generated by the Hamiltonian $E$ ) and the rotation flow (generated by the Hamiltonian $J$ ). The second measure is a usual microlocal/semiclassical measure whereas the first one is a less usual operator-valued measure taking into account non-oscillatory phenomena. We prove that both second-microlocal measures enjoy additional invariance properties: the first one is invariant by the rotation flow, whereas the second one propagates through a Heisenberg equation on the circle. This translates, respectively, into Theorem 2.4 (ii) and (iii).

This program was already completed in [Mac10, AM14, AFKM14] for the Schrödinger equation on flat tori, but carrying it out in the disk induces considerable additional difficulties. Our phase space does not directly come equipped with its action-angle coordinates, so that we need first to change variables. This requires in particular to build a Fourier Integral Operator to switch from $(z, \xi)$-variables to action-angle coordinates. These coordinates are very nice to understand the dynamics and are necessary to perform the second microlocalization, but they are extremely nasty to treat the boundary condition, for which the use of polar coordinates is more suitable. It seems that we cannot avoid having to go back and forth between the two sets of coordinates. Our approach to that particular technical aspect is inspired by [GL93]; however, the second-microlocal nature of the problem requires to perform the asymptotic expansions of [GL93] one step further.

\subsection{Relations to other works.}

1.6.1. Regularity of semiclassical measures. This work pertains to the longstanding study of the so-called "quantum-classical correspondence", which aims at understanding the links between high frequency solutions of the Schrödinger equation and the dynamics of the underlying billiard flow (see for instance the survey article [AM12]).

More precisely, it is concerned with a case of completely integrable billiard flow. This particular dynamical situation has already been addressed in [Mac10] and [AM14] in the case of flat tori, and in [AFKM14] for more general integrable systems (without boundary). These three papers use in a central way a "second microlocalization" to understand the concentration of measures on invariant tori. The main tools are second-microlocal semiclassical measures, introduced in the local Euclidean setting in [Nie96, FK00a, FK00b, FKG02, Mil96, Mil97], and defined in [Mac10, AM14, AFKM14] as global objects.

On the sphere $\mathbb{S}^{d}$, or more generally, on a manifold with periodic geodesic flow, the situation is radically different. The geodesic flow for this type of geometries is still completely integrable, but it is known [Mac09] (see also [JZ99, Mac08, AM10] for the special case of eigenfunctions) that every invariant measure is a Wigner measure; those are not necessarily absolutely continuous when projected in the position space. The difference with the previous situation is that the underlying dynamical system, though completely integrable, is degenerate. What was evidenced in [AFKM14] is that a sufficient and necessary for the absolute continuity of Wigner measures, is that the hamiltonian be a strictly convex/concave function of the action variables - a condition that is even stronger than non-degeneracy. In the case of the disk, the complete integrability of the billiard flow on $\mathbb{D}$ degenerates on the boundary. There, both actions coincide, which allows for the concentration of solutions on the invariant torus at the boundary (as was the case with the aforementioned whispering gallery modes).

Note that on the torus and on the disk, it remains an open question to fully characterize the set of Wigner measures associated to sequences of solutions to the time-dependent Schrödinger 
equation. In the case of flat tori, the papers [Jak97, AMJ12] provide additional information about the regularity of the measures.

1.6.2. Observability of the Schrödinger equation. Since the pioneering work of Lebeau [Leb92], it is known that observability inequalities like (1.5)-(1.6) always hold if all trajectories of the billiard enter the observation region $\Omega$ or $\Gamma$ in finite time. However, since [Har89, Jaf90], we know that this strong geometric control condition is not necessary: (1.5) holds on the two-torus as soon as $\Omega \neq \emptyset$; for different proofs and extensions of this result see [Kom92, BZ04, Mac11, BZ12, BBZ13, AM14]. These properties seem to deeply depend on the global dynamics of the billiard flow.

On manifolds with periodic geodesic flow, it is necessary that $\bar{\Omega}$ meets all geodesics for an observation inequality as (1.5) to hold [Mac11]. This is due to the strong stability properties of the geodesic flow.

To our knowledge, apart from the case of flat tori, few results are known concerning the observability of the Schrödinger equation in situations where the geometric control condition fails. The paper [AFKM14] extends [AM14] to general completely integrable systems under a convexity assumption for the hamiltonian. Note also that the boundary observability (1.6) holds in the square if (and only if) the observation region $\Gamma$ contains both a horizontal and a vertical nonempty segments [RTTT05]. Finally, for chaotic systems, the observability inequality (1.5) is also valid on manifolds with negative curvature if the set of uncontrolled trajectories is sufficiently small [Ana08, AR12].

Our Theorems 1.2 and 1.3 provide necessary and sufficient conditions for the observability of the Schrödinger group on the disk. This is clear in the case of boundary observability, and in the case of internal observability, if $\Omega \subset \mathbb{D}$ is such that $\Omega \cap \partial \mathbb{D}=\emptyset$, the observability inequality (1.5) fails. When $V=0$ this comes from the existence of whispering-gallery modes, see Section 1.3, and this remains true for any $V$, as proved in Remark 2.11.

Let us conclude this introduction with a few more remarks.

Remark 1.5. In this article, we only treat the case of Dirichlet boundary conditions. The extension of our method to the Neumann or mixed boundary condition deserves further investigation.

Remark 1.6. Let us comment on the regularity required on the potential $V$. Arguments developed in [AM14] show that all the results of this paper could actually be weakened to $V \in C^{0}(\mathbb{R} \times \overline{\mathbb{D}} ; \mathbb{R})$ or even to the case where $V$ is continuous outside a set of zero measure. Corollary 1.1 in fact also holds for any $V \in L_{\mathrm{loc}}^{2}\left(\mathbb{R} ; \mathcal{L}\left(L^{2}(\mathbb{D})\right)\right)$, and in particular for any bounded potentials. See also Remark 2.5 below.

Remark 1.7. Our results directly yield a polynomial decay rate for the energy of the damped wave equation $\left(\partial_{t}^{2}-\Delta+b(z) \partial_{t}\right) u=0$ with Dirichlet Boundary conditions on the disk. More precisely, [AL14, Theorem 2.3] and Theorem 1.2 imply that if $b \geq 0$ is positive on an open set $\Omega$ such that $\Omega \cap \partial \mathbb{D} \neq \emptyset$, then the $H_{0}^{1} \times L^{2}$ norm of solutions decays at rate $1 / \sqrt{t}$ for data in $\left(H^{2} \cap H_{0}^{1}\right) \times H_{0}^{1}$. This rate is better than the a priori logarithmic decay rate given by the Lebeau theorem [Leb96]. The latter is however optimal if $\operatorname{supp}(b) \cap \partial \mathbb{D}=\emptyset$ as a consequence of the whispering gallery phenomenon (see e.g. [LR97]).

Acknowledgement. We thank Patrick Gérard for his continued encouragement and very helpful discussions.

NA and ML are supported by the Agence Nationale de la Recherche under grant GERASIC ANR-13-BS01-0007-01.

NA acknowledges support by the National Science Foundation under agreement no. DMS 1128155, by the Fernholz foundation, by Agence Nationale de la Recherche under the grant ANR09-JCJC-0099-01, and by Institut Universitaire de France. Any opinions, findings and conclusions or recommendations expressed in this material are those of the authors and do not necessarily reflect the views of the NSF.

FM takes part into the visiting faculty program of ICMAT and is partially supported by grants MTM2013-41780-P (MEC) and ERC Starting Grant 277778. 


\section{The Structure Theorem}

In this section, we give the main definitions used in the article and state our main structure theorems. We first define microlocal and semiclassical Wigner measures (which are the main objects discussed in the paper) in Section 2.1. We then briefly describe the billiard flow and introduce adapted action-angle coordinates in Section 2.2. This allows us to formulate our main results (Sections 2.4 and 2.5), both in the semiclassical (Theorem 2.4) and in the microlocal (Theorem 2.6) framework. Next, in Section 2.7, we define various measures at the boundary of the disk, that will be useful in the proofs, and explain their links with the Wigner measures in the interior.

2.1. Wigner measures: microlocal versus semiclassical point of view. Let $T^{*} \mathbb{R}^{2}=\mathbb{R}^{2} \times \mathbb{R}^{2}$ be the cotangent bundle over $\mathbb{R}^{2}$, and $T^{*} \mathbb{R}=\mathbb{R} \times \mathbb{R}$ be the cotangent bundle over $\mathbb{R}$. We shall denote by $z \in \mathbb{R}^{2}$ (resp. $t \in \mathbb{R}$ ) the space (resp. time) variable and $\xi \in \mathbb{R}^{2}$ (resp. $H \in \mathbb{R}$ ) the associated frequency.

Our main results can be formulated in two different and complementary settings. We first introduce the symbol class needed to formulate their microlocal version, allowing to define microlocal Wigner distributions. We then define semiclassical Wigner distributions and briefly compare these two objects.

Definition 2.1. Let us call $\mathcal{S}_{0}$ the space of functions $a \in C^{\infty}\left(T^{*} \mathbb{R}^{2} \times T^{*} \mathbb{R}\right), a(z, \xi, t, H)$ such that

(a) a is compactly supported in the variables $z, t$.

(b) a is homogeneous at infinity in $(\xi, H)$ in the following sense: there exists $R_{0}>0$ such that

$$
a(z, \xi, t, H)=a\left(z, \lambda \xi, t, \lambda^{2} H\right), \quad \text { for }|\xi|^{2}+|H|>R_{0} \text { and } \lambda \geq 1 .
$$

Equivalently, there is $a_{\mathrm{hom}} \in C^{\infty}\left(T^{*} \mathbb{R}^{2} \times T^{*} \mathbb{R} \backslash\{(\xi, H)=(0,0)\}\right)$ satisfying (2.1) for all $\lambda>0$, such that

$$
a(z, \xi, t, H)=a_{\mathrm{hom}}(z, \xi, t, H), \quad \text { for }|\xi|^{2}+|H|>R_{0} .
$$

Such a homogeneous function $a_{\mathrm{hom}}$ is entirely determined by its restriction to the set $\left\{|\xi|^{2}+2|H|=2\right\} \subset \mathbb{R}^{2} \times \mathbb{R}$, which is homeomorphic to a 2-dimensional sphere $\mathbb{S}^{2}$. Thus we may (and will, when convenient) identify $a_{\mathrm{hom}}$ with a function in the space $C^{\infty}\left(\mathbb{R}_{z}^{2} \times \mathbb{R}_{t} \times \mathbb{S}_{\xi, H}^{2}\right)$.

Note that the different homogeneity with respect to the $H$ and $\xi$ variables is adapted to the scaling of the Schrödinger operator.

Let $\left(u_{n}^{0}\right)$ be a sequence in $L^{2}(\mathbb{D})$, such that $\left\|u_{n}^{0}\right\|_{L^{2}(\mathbb{D})}=1$ for all $n$. For $z \in \mathbb{D}$ and $t \in \mathbb{R}$ we denote $u_{n}(z, t)=U_{V}(t) u_{n}^{0}(z)$. In what follows (e.g. in formula (2.2) below), we shall systematically extend the functions $u_{n}$, a priori defined on $\mathbb{D}$, by the value 0 outside $\mathbb{D}$ as done in [GL93], where semiclassical Wigner measures for boundary value problems were first considered. The extended sequence now satisfies the equation

$$
\left(-\frac{1}{2} \Delta+V-D_{t}\right) u_{n}=\frac{1}{2} \frac{\partial u_{n}}{\partial n} \otimes \delta_{\partial \mathbb{D}}, \quad(z, t) \in \mathbb{R}^{2} \times \mathbb{R},
$$

where $\Delta$ denotes the Laplacian on $\mathbb{R}^{2}$. Remark that the term $\left.\frac{\partial u_{n}}{\partial n}\right\rceil_{\partial \mathbb{D}}$ has no straightforward meaning at this level of regularity. We shall see below how to give a signification to this equation, both in the semiclassical (see Remark 2.3) and in the microlocal (see Section 2.8.3) settings).

The microlocal Wigner distributions associated to $\left(u_{n}\right)$ act on symbols $a \in \mathcal{S}_{0}$ by

$$
W_{u_{n}}(a):=\left\langle u_{n}, \mathrm{Op}_{1}(a) u_{n}\right\rangle_{L^{2}\left(\mathbb{R}_{z}^{2} \times \mathbb{R}_{t}\right)},
$$

where $\operatorname{Op}_{1}(a)=a\left(z, D_{z}, t, D_{t}\right)$ (with the standard notation $D=-i \partial$ ) is a pseudodifferential operator defined by the standard quantization procedure. In what follows, $\operatorname{Op}_{\epsilon}(a)=a\left(z, \epsilon D_{z}, t, \epsilon D_{t}\right)$ will stand for the operator acting on $L^{2}\left(\mathbb{R}^{2} \times \mathbb{R}\right)$ by:

$$
\left(\mathrm{Op}_{\epsilon}(a) u\right)(z, t)=\frac{1}{(2 \pi \epsilon)^{3}} \int_{\mathbb{R}^{2} \times \mathbb{R}} \int_{\mathbb{R}^{2} \times \mathbb{R}} e^{\frac{i \xi \cdot\left(z-z^{\prime}\right)+i H\left(t-t^{\prime}\right)}{\epsilon}} a(z, \xi, t, H) u\left(z^{\prime}, t^{\prime}\right) d z^{\prime} d t^{\prime} d \xi d H .
$$


Usual estimates on pseudodifferential operators imply that $W_{u_{n}}$ is well defined, and forms a bounded sequence in $\mathcal{S}_{0}^{\prime}$. The main goal of this article is to understand properties of weak limits of $\left(W_{u_{n}}\right)$ that are valid for any sequence of initial conditions $\left(u_{n}^{0}\right)$.

The problem also has a semiclassical variant. In this version, one considers $a \in C_{c}^{\infty}\left(T^{*} \mathbb{R}^{2} \times\right.$ $T^{*} \mathbb{R}$ ), a real parameter $h>0$, and one defines the semiclassical Wigner distributions at scale $h$ by

$$
W_{u_{n}}^{h}(a):=\left\langle u_{n}, \mathrm{Op}_{1}\left(a\left(z, h \xi, t, h^{2} H\right)\right) u_{n}\right\rangle_{L^{2}\left(\mathbb{R}_{z}^{2} \times \mathbb{R}_{t}\right)},
$$

where $\operatorname{Op}_{1}\left(a\left(z, h \xi, t, h^{2} H\right)\right)=a\left(z, h D_{z}, t, h^{2} D_{t}\right)=\mathrm{Op}_{h}(a(z, \xi, t, h H))$, see (2.3). Note that this scaling relation is the natural one for solutions of (1.1), and its interest will be made clear below. Again $W_{u_{n}}^{h}$ is well defined, and forms a bounded sequence in $\mathcal{D}^{\prime}\left(T^{*} \mathbb{R}^{2} \times T^{*} \mathbb{R}\right)$ if $h$ stays bounded. This formulation is most meaningful if the parameter $h=h_{n}$ is chosen in relation with the typical scale of oscillation of our sequence of initial conditions $\left(u_{n}^{0}\right)$.

Definition 2.2. Given a bounded sequence $\left(w_{n}\right)$ in $L^{2}(\mathbb{D})$, we shall say that it is $\left(h_{n}\right)$-oscillating from above (resp. $\left(h_{n}\right)$-oscillating from below) if the sequence $\left(w_{n}\right)$ extended by zero outside of $\mathbb{D}$ satisfies:

(resp.

$$
\lim _{R \rightarrow \infty} \limsup _{n \rightarrow \infty} \int_{|\xi| \geq R / h_{n}}\left|\widehat{w_{n}}(\xi)\right|^{2} d \xi=0
$$

$$
\left.\lim _{\epsilon \rightarrow 0} \limsup _{n \rightarrow \infty} \int_{|\xi| \leq \epsilon / h_{n}}\left|\widehat{w_{n}}(\xi)\right|^{2} d \xi=0 \quad\right)
$$

where $\widehat{w_{n}}$ is the Fourier transform of $w_{n}$ on $\mathbb{R}^{2}$.

The property of being $\left(h_{n}\right)$-oscillating from above is only relevant if $h_{n} \longrightarrow 0$; if $u_{n}^{0}$ is $\left(h_{n}\right)$ oscillating for $\left(h_{n}\right)$ bounded away from 0 , the (extended) sequence $\left(u_{n}^{0}\right)$ is compact in $L^{2}$ and the structure of the accumulation points of $\left(W_{u_{n}}^{h_{n}}\right)$ is trivial. Therefore, we shall always assume that $h_{n} \longrightarrow 0$. Note that one can always find $\left(h_{n}\right)$ tending to zero such that $\left(u_{n}^{0}\right)$ is $h_{n}$-oscillating from above (to see that, note that for fixed $n$ one may choose $h_{n}$ such that $\int_{|\xi| \geq 1 / h_{n}}\left|\widehat{u_{n}^{0}}(\xi)\right|^{2} d \xi \leq n^{-1}$ ). However, the choice of the sequence $h_{n}$ is by no means unique $\left(h_{n}\right.$-oscillating sequences are also $h_{n}^{\prime}$-oscillating as soon as $h_{n}^{\prime} \leq h_{n}$ ), although in many cases there is a natural scale $h_{n}$ given by the problem under consideration.

One can find $\left(h_{n}^{\prime}\right)$ such that $\left(u_{n}^{0}\right)$ is $h_{n}^{\prime}$-oscillating from below if and only if the extended $\left(u_{n}^{0}\right)$ converges to 0 weakly in $L^{2}\left(\mathbb{R}^{2}\right)$. It is not always possible to find a common $\left(h_{n}\right)$ such that $\left(u_{n}^{0}\right)$ is $h_{n}$-oscillating both from above and below (see [Gér98] for an example of a sequence with this behavior). However, when it is the case, the semiclassical Wigner distributions contain more information that the microlocal ones (see Section 2.6). On the other hand, if no $h_{n}$ exists such that $\left(u_{n}^{0}\right)$ is $h_{n}$-oscillating from above and below, the accumulation points of $W_{u_{n}}^{h_{n}}$ may fail to describe completely the asymptotic phase-space distribution of the sequence $\left(u_{n}\right)$, either because some mass will escape to $|\xi|=\infty$ or because the fraction of the mass going to infinity at a rate slower that $h_{n}^{-1}$ will give a contribution concentrated on $\xi=0$. In those cases, the microlocal formulation is still able to describe the asymptotic distribution of the sequence on the reduced phase-space $\mathbb{R}_{z}^{2} \times \mathbb{R}_{t} \times \mathbb{S}_{\xi, H}^{2}$.

This is one of the motivations that has lead us to study both points of view, semiclassical and microlocal.

2.2. The billiard flow. Microlocal or semiclassical analysis provide a connection between the Schrödinger equation and the billiard on the underlying phase space. In this section we first clarify what we mean by "billiard flow" in the disk. The phase space associated with the billiard flow on the disk can be defined as a quotient of $\overline{\mathbb{D}} \times \mathbb{R}^{2}$ (position $\times$ frequency). We first define the symmetry with respect to the line tangent to the circle $\partial \mathbb{D}$ at $z \in \partial \mathbb{D}$ by

$$
\sigma_{z}(\xi)=\xi-2(z \cdot \xi) z, \quad \sigma(z, \xi)=\left(z, \sigma_{z}(\xi)\right), \quad z \in \partial \mathbb{D} .
$$

Then, we work on the quotient space

$$
\mathbb{W}=\overline{\mathbb{D}} \times \mathbb{R}^{2} / \sim \quad \text { where }(z, \xi) \sim \sigma(z, \xi) \text { for }|z|=1 .
$$


We denote by $\pi$ the canonical projection $\overline{\mathbb{D}} \times \mathbb{R}^{2} \rightarrow \mathbb{W}$ which maps a point $(z, \xi)$ to its equivalence class modulo $\sim$. Note that $\pi$ is one-one on $\mathbb{D} \times \mathbb{R}^{2}$, so that $\mathbb{D} \times \mathbb{R}^{2}$ may be seen as a subset of $\mathbb{W}$.

A function $a \in C^{0}(\mathbb{W})$ can be identified with the function $\tilde{a}=a \circ \pi \in C^{0}\left(\overline{\mathbb{D}} \times \mathbb{R}^{2}\right)$ satisfying $\tilde{a}(z, \xi)=\tilde{a} \circ \sigma(z, \xi)$ for $(z, \xi) \in \partial \mathbb{D} \times \mathbb{R}^{2}$.

The billiard flow $\left(\phi^{\tau}\right)_{\tau \in \mathbb{R}}$ on $\mathbb{W}$ is the (uniquely defined) action of $\mathbb{R}$ on $\mathbb{W}$ such that the map $(\tau, z, \xi) \mapsto \phi^{\tau}(z, \xi)$ is continuous on $\mathbb{R} \times \mathbb{W}$, satisfies $\phi^{\tau+\tau^{\prime}}=\phi^{\tau} \circ \phi^{\tau^{\prime}}$, and such that

$$
\phi^{\tau}(z, \xi)=(z+\tau \xi, \xi)
$$

whenever $z \in \mathbb{D}$ and $z+\tau \xi \in \mathbb{D}$.

In order to understand how the completely integrable dynamics of the flow $\phi^{\tau}$ influences the structure of Wigner measures, we need to introduce coordinates adapted to this dynamics. We denote by

$$
\Phi:(s, \theta, E, J) \mapsto\left(x, y, \xi_{x}, \xi_{y}\right),
$$

the set of "action-angle" coordinates for the billiard flow (see also Section 3.1), defined by:

$$
\left\{\begin{array}{l}
x=\frac{J}{E} \cos \theta-s \sin \theta \\
y=\frac{J}{E} \sin \theta+s \cos \theta \\
\xi_{x}=-E \sin \theta \\
\xi_{y}=E \cos \theta
\end{array}\right.
$$

These coordinates are illustrated in Figure 1. The inverse map is given by the formulas

$$
\left\{\begin{array}{l}
E=\sqrt{\xi_{x}^{2}+\xi_{y}^{2}}, \text { (velocity) } \\
J=x \xi_{y}-y \xi_{x}, \text { (angular momentum) } \\
\theta=-\arctan \left(\frac{\xi_{x}}{\xi_{y}}\right),(\text { angle of } \xi \text { with the vertical) } \\
s=-x \sin \theta+y \cos \theta,\left(\text { abscissa of }(x, y) \text { along the line }\left(\frac{J}{E} \cos \theta, \frac{J}{E} \sin \theta\right)+\mathbb{R} \xi\right) .
\end{array}\right.
$$

In other words, we have:

$$
\left\{\begin{array}{l}
E=|\xi|, \\
J=z \cdot \xi^{\perp}, \\
s=z \cdot \frac{\xi}{|\xi|},
\end{array}\right.
$$

where $\xi^{\perp}:=\left(\xi_{y},-\xi_{x}\right)$, and

$$
\left\{\begin{array}{l}
\xi=\left(\xi_{x}, \xi_{y}\right)=E(-\sin (\theta), \cos (\theta)) \\
z=(x, y)=s(-\sin (\theta), \cos (\theta))+\frac{J}{E}(\cos (\theta), \sin (\theta))=\left(z \cdot \frac{\xi}{|\xi|}\right) \frac{\xi}{|\xi|}+\left(z \cdot \frac{\xi^{\perp}}{|\xi|}\right) \frac{\xi^{\perp}}{|\xi|} .
\end{array}\right.
$$

Note that the velocity $E$ and the angular momentum $J$ are preserved along the free transport flow in $\mathbb{R}^{2} \times \mathbb{R}^{2}$, but also along $\phi^{\tau}$; the variables $s$ and $\theta$ play the role of "angle" coordinates. We call $\alpha=-\arcsin \left(\frac{J}{E}\right)$ the angle that a billiard trajectory makes with the normal to the circle, when it hits the boundary (see Figure 2). The quantity $\alpha$ is preserved by the billiard flow.

Let us denote $T_{(E, J)}$ the level sets of the pair $(E, J)$, namely

$$
T_{(E, J)}=\left\{(z, \xi) \in \overline{\mathbb{D}} \times \mathbb{R}^{2}:\left(|\xi|, z \cdot \xi^{\perp}\right)=(E, J)\right\} .
$$

For $E \neq 0$ let us denote $\lambda_{E, J}$ the probability measure on $T_{(E, J)}$ that is both invariant under the billiard flow and invariant under rotations. In the coordinates $(s, \theta, E, J)$, we have

$$
\lambda_{E, J}(d s, d \theta)=c(E, J) d s d \theta, \quad c(E, J)=\left(\int_{T(E, J)} d s d \theta\right)^{-1}>0 .
$$

Note that for $E \neq 0$ and $\alpha \in \pi \mathbb{Q}$ the billiard flow is periodic on $T_{(E, J)}$ whereas $\alpha \notin \pi \mathbb{Q}$ corresponds to trajectories that hit the boundary on a dense set. More precisely, if $\alpha \notin \pi \mathbb{Q}$ then the billiard flow restricted to $T_{(E, J)}$ has a unique invariant probability measure, namely $\lambda_{E, J}$. 


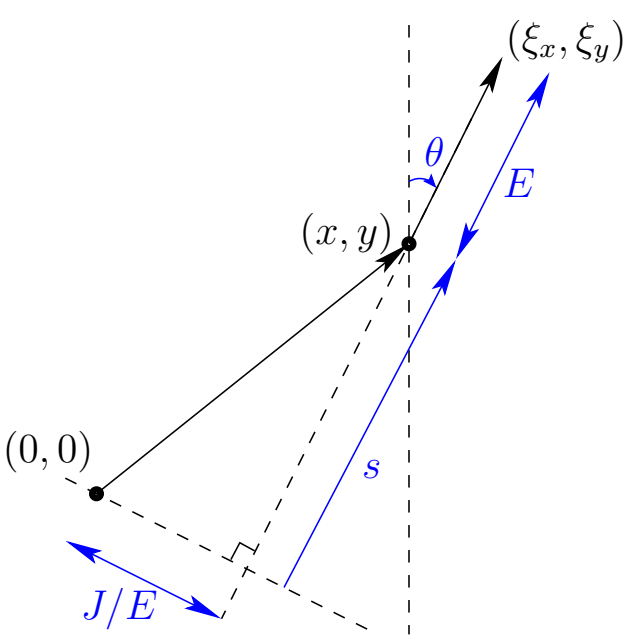

Figure 1. Action-angle coordinates for the billiard flow on the disk.

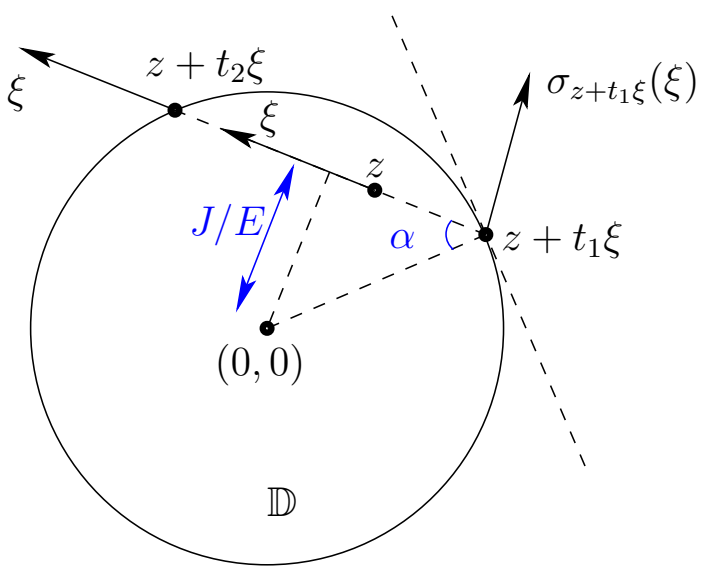

Figure 2. Angle $\alpha$.

2.3. Standard facts about Wigner measures. We start formulating the question and results in a semiclassical framework: we have a parameter $h_{n}$ going to 0 , meant to represent the typical scale of oscillation of our sequence of initial conditions $\left(u_{n}^{0}\right)$.

We simplify the notation by writing $h=h_{n}, u_{h}^{0}=u_{n}^{0}$. We will always assume that the functions $u_{h}^{0}$ are normalized in $L^{2}(\mathbb{D})$. We define $u_{h}(z, t)=U_{V}(t) u_{h}^{0}(z)$ (the reader should be aware that $u_{h}$ satisfies the classical Schrödinger Equation (1.1); the index $h$ only reminds its oscillation scale). Since this is a function on $\mathbb{D} \times \mathbb{R}$ it is natural to do a frequency analysis both in $z$ and $t$. Recall that we keep the notation $u_{h}$ after the extension by zero outside $\mathbb{D}$. Recall that the semiclassical Wigner distribution associated to $u_{h}$ (at scale $h$ ) is a distribution on the cotangent bundle $T^{*} \mathbb{R}^{2} \times T^{*} \mathbb{R}=\mathbb{R}_{z}^{2} \times \mathbb{R}_{\xi}^{2} \times \mathbb{R}_{t} \times \mathbb{R}_{H}$, defined by

$$
W_{u_{h}}^{h}: a \mapsto\left\langle u_{h}, \mathrm{Op}_{1}\left(a\left(z, h \xi, t, h^{2} H\right)\right) u_{h}\right\rangle_{L^{2}\left(\mathbb{R}^{2} \times \mathbb{R}\right)}, \quad \text { for all } a \in C_{c}^{\infty}\left(T^{*} \mathbb{R}^{2} \times T^{*} \mathbb{R}\right) .
$$

The scaling $\mathrm{Op}_{1}\left(a\left(z, h \xi, t, h^{2} H\right)\right)$ is performed in order to capture all the information whenever $u_{h}$ is $h$-oscillating from above (if $u_{h}$ is not $h$-oscillating from above, the discussion below remains entirely valid but part of the information about $u_{h}(z, t)$ is lost when studying $W_{u_{h}}^{h}(a)$ ). Under this assumption, if $a$ is a function on $T^{*} \mathbb{R}^{2} \times T^{*} \mathbb{R}$ that depends only on $(z, t)$, we have

$$
W_{u_{h}}^{h}(a)=\int_{\mathbb{D}} a(z, t)\left|u_{h}(z, t)\right|^{2} d z d t .
$$


When no confusion arises, we shall denote $W_{h}$ for $W_{u_{h}}^{h}$.

By standard estimates on the norm of $\operatorname{Op}_{1}(a)$, it follows that $W_{h}$ belongs to $\mathcal{D}^{\prime}\left(T^{*} \mathbb{R}^{2} \times T^{*} \mathbb{R}\right)$, and is uniformly bounded in that space as $h \longrightarrow 0^{+}$. Thus, one can extract subsequences that converge in the weak-* topology of $\mathcal{D}^{\prime}\left(T^{*} \mathbb{R}^{2} \times T^{*} \mathbb{R}\right)$. In other words, after possibly extracting a subsequence, we have

$$
W_{h}(a) \underset{h \longrightarrow 0}{\longrightarrow} \mu_{s c}(a)
$$

for all $a \in C_{c}^{\infty}\left(T^{*} \mathbb{R}^{2} \times T^{*} \mathbb{R}\right)$.

In this paper such a measure $\mu_{s c}$ will be called a semiclassical Wigner measure, or in short semiclassical measure, associated with the initial conditions $\left(u_{h}^{0}\right)$ and the scale $h$.

Remark 2.3. Fix $R>0$; Remark C.3 below tells us that in order to compute the restriction of $\mu_{s c}$ to the set $\{|H|<R\}$ we may, without loss of generality, assume that $u_{h}^{0} \in H_{0}^{1}(\mathbb{D})$ and $\left\|\nabla u_{h}^{0}\right\|_{L^{2}(\mathbb{D})}=O_{R}\left(h^{-1}\right)$. In that case Proposition C.1 says that the boundary data $h \partial_{n}\left(U_{V}(t) u_{h}^{0}\right)$ form a bounded sequence in $L_{\text {loc }}^{2}(\mathbb{R} \times \partial \mathbb{D})$. We can work under these assumptions when necessary. This determines $\mu_{\text {sc }}$ completely as $R$ is arbitrary.

It follows from standard properties of pseudodifferential operators that the limit $\mu_{s c}$ in (2.9) has the following properties:

- $\mu_{s c}$ is a nonnegative measure, of the form $\mu_{s c}(d z, d \xi, d t, d H)=\mu_{s c}(d z, d \xi, t, d H) d t$ where $t \mapsto \mu_{s c}(t) \in L^{\infty}\left(\mathbb{R}_{t} ; \mathcal{M}_{+}\left(T^{*} \mathbb{R}^{2} \times \mathbb{R}_{H}\right)\right)$. Moreover, for a.e. $t \in \mathbb{R}, \mu_{s c}(t)$ is supported in $\left\{|\xi|^{2}=2 H\right\} \cap\left(\overline{\mathbb{D}} \times \mathbb{R}^{2} \times \mathbb{R}_{H}\right)$. See [GMMP97, LP93] for a proof of nonnegativity; the time regularity and the localization of the support are shown in Proposition D.1.

- From the normalization of $u_{h}^{0}$ in $L^{2}$, we have for a.e. $t$ :

$$
\int_{\overline{\mathbb{D}} \times \mathbb{R}^{2} \times \mathbb{R}} \mu_{s c}(d z, d \xi, t, d H) \leq 1,
$$

the inequality coming from the fact that $\overline{\mathbb{D}} \times \mathbb{R}^{2} \times \mathbb{R}$ is not compact, and that there may be an escape of mass to infinity (however, if $u_{h}^{0}$ is $h$-oscillating from above, escape of mass does not occur and we have $\left.\int_{\overline{\mathbb{D}} \times \mathbb{R}^{2} \times \mathbb{R}} \mu_{s c}(d z, d \xi, t, d H)=1\right)$.

- The standard quantization enjoys the following property:

$$
\left[-\frac{i h}{2} \Delta, \mathrm{Op}_{h}(a)\right]=\mathrm{Op}_{h}\left(\xi \cdot \partial_{z} a-\frac{i h}{2} \Delta_{z} a\right),
$$

where $\Delta$ is the Laplacian on $\mathbb{R}^{2}$. From this identity, one can show that

$$
\int_{\overline{\mathbb{D}} \times \mathbb{R}^{2} \times \mathbb{R}} \xi \cdot \partial_{z} a \mu_{s c}(d z, d \xi, t, d H)=0
$$

for a.e. $t$ and for every smooth $a$ such that $a(z, \xi, t, H)=a\left(z, \sigma_{z}(\xi), t, H\right)$ for $|z|=1$. Equivalently,

$$
\int_{\overline{\mathbb{D}} \times \mathbb{R}^{2} \times \mathbb{R}} a \circ \phi^{\tau} \circ \pi(z, \xi, t, H) \mu_{s c}(d z, d \xi, t, d H)=\int_{\overline{\mathbb{D}} \times \mathbb{R}^{2} \times \mathbb{R}} a \circ \pi(z, \xi, t, H) \mu_{s c}(d z, d \xi, t, d H)
$$

for every $a \in C^{0}(\mathbb{W}), \tau \in \mathbb{R}$ - where $\phi^{\tau}$ is the billiard flow in the disk and $\pi: \overline{\mathbb{D}} \times \mathbb{R}^{2} \rightarrow \mathbb{W}$ the canonical projection, defined in Section 2.2. In other words, $\pi_{*} \mu$ is an invariant measure of the billiard flow.

We refer to Section 2.8 for a more general version of (2.10) (as formulated in [GL93], see also [RZ09]) involving a measure associated to boundary traces.

2.4. The structure theorem: semiclassical formulation. Now comes our central result, giving the structure of semiclassical measures arising as weak-* limits of sequences $\left(W_{h}\right)$ associated to solutions of (1.1). As a by-product it clarifies the dependence of $\mu_{s c}(t, \cdot)$ on the time parameter $t$. It was already noted in [Mac09] that the dependence of $\mu_{s c}(t, \cdot)$ on the sequence of initial conditions is a subtle issue.

The statement of Theorem 2.4 is technical and needs introducing some notation. We first restrict our attention to the case where the initial conditions $\left(u_{h}^{0}\right)$ are $h$-oscillating from below, 
or equivalently $\mu_{s c}$ does not charge $\{\xi=0\}$ (otherwise, the restriction of $\mu_{s c}$ to $\{\xi=0\}$ will be better understood at the end of Section 2.6).

The notation $(s, \theta, E, J), \alpha$ is as in Section 2.2. Here we restrict our discussion to $E \neq 0$. For each $\alpha_{0} \in \pi \mathbb{Q} \cap(-\pi / 2, \pi / 2)$ we will introduce a flow $\left(\phi_{\alpha_{0}}^{\tau}\right)$ on the billiard phase space $\mathbb{W}$, all of whose orbits are periodic (Lemma 3.4). It coincides with the billiard flow on the set

$$
\mathcal{I}_{\alpha_{0}}=\left\{(s, \theta, E, J) \in \Phi^{-1}\left(\overline{\mathbb{D}} \times \mathbb{R}^{2}\right), J=-\sin \alpha_{0} E\right\}=\left\{\alpha=\alpha_{0}\right\},
$$

which is the union of all the lagrangian manifolds $T_{(E, J)}$ with $J=-\sin \alpha_{0} E$. If $a$ is a function on $\mathbb{W}$, we shall denote by $\langle a\rangle_{\alpha_{0}}$ its average along the orbits of $\phi_{\alpha_{0}}^{\tau}$ (actually, $\langle a\rangle_{\alpha_{0}}$ is well defined even if $a$ is not symmetric with respect to the boundary, since the set of hitting times of the boundary has measure 0$)$. In the coordinates $(s, \theta, E, J)$, this is a function whose restriction to $\mathcal{I}_{\alpha_{0}}$ does not depend on $s$.

We will denote by

$$
m_{a}^{\alpha_{0}}(s, E, t, H)
$$

the operator on $L_{\text {loc }}^{2}\left(\mathbb{R}_{\theta}\right)$ acting by multiplication by the function

$$
a\left(\Phi\left(s, \theta, E,-E \sin \alpha_{0}\right), t, H\right) .
$$

If $a$ is a symmetric function (i.e. a function on $\mathbb{W}$ ), remark that $m_{\langle a\rangle_{\alpha_{0}}}^{\alpha_{0}}$ does not depend on the variable $s$. For our potential $V$, the function $\langle V\rangle_{\alpha_{0}} \circ \Phi$ depends only on $\theta$ (and $t$ if $V$ is time-dependent).

Given $\omega \in \mathbb{R} / 2 \pi \mathbb{Z}$, we denote by $U_{\alpha_{0}, \omega}(t)$ the unitary propagator of the equation

$$
-\cos ^{2} \alpha_{0} D_{t} v(t, \theta)+\left(-\frac{1}{2} \partial_{\theta}^{2}+\cos ^{2} \alpha_{0}\langle V\rangle_{\alpha_{0}} \circ \Phi\right) v(t, \theta)=0
$$

acting on the Hilbert space

$$
\mathcal{H}_{\omega}=\left\{v \in L_{\text {loc }}^{2}(\mathbb{R}): v(\theta+2 \pi)=v(\theta) e^{i \omega} \text {, for a.e. } \theta \in \mathbb{R}\right\},
$$

i.e. with Floquet-periodic condition. In the statements below, each $\mathcal{H}_{\omega}$ is identified with $L^{2}(0,2 \pi)$ by taking restriction of functions to $(0,2 \pi)$.

Theorem 2.4. Let $\left(u_{h}^{0}\right)$ be a family of initial data, assumed to be h-oscillating from below. There exists a subsequence of $W_{h}$ converging weakly-* to a positive measure $\mu_{s c}$ that can be decomposed into a countable sum of non-negative measures:

$$
\mu_{s c}=\nu_{L e b}+\sum_{\alpha_{0} \in \pi \mathbb{Q} \cap[-\pi / 2, \pi / 2]} \nu_{\alpha_{0}},
$$

satisfying:

(i) Each of the measures in the decomposition above is carried by the set $\left\{H=\frac{E^{2}}{2}\right\}$ and is invariant under the billiard flow.

(ii) The measure $\nu_{L e b}$ is constant in $t$; $\nu_{L e b}$ is of the form $\int_{E>0,|J| \leq E} \lambda_{E, J} d \nu^{\prime}(E, J) d t$ for some nonnegative measure $\nu^{\prime}$ on $\mathbb{R}^{2}$. In other words $\nu_{\text {Leb }}$ is a combination of Lebesgue measures on the invariant "tori" $T_{(E, J)}$.

(iii) For every $\alpha_{0} \in \pi \mathbb{Q} \cap(-\pi / 2, \pi / 2)$, the measure $\nu_{\alpha_{0}}$ is carried by the set $\mathcal{I}_{\alpha_{0}} \cap\left\{H=E^{2} / 2\right\}$ and is characterized by

$$
\int_{\mathcal{I}_{\alpha_{0}}} a d \nu_{\alpha_{0}}=\int_{\mathcal{I}_{\alpha_{0}}} \operatorname{Tr}_{L^{2}(0,2 \pi)}\left(m_{\langle a\rangle_{\alpha_{0}}}^{\alpha_{0}} \sigma_{\alpha_{0}}\right) d \ell_{\alpha_{0}}, \quad \text { for all } a \in C_{c}^{\infty}\left(T^{*} \mathbb{R}^{2} \times T^{*} \mathbb{R}\right),
$$

where $\ell_{\alpha_{0}}(d \omega, d E, d H, d t)$ is a nonnegative measure on $\mathbb{R} / 2 \pi \mathbb{Z} \times \mathbb{R}_{E} \times \mathbb{R}_{H} \times \mathbb{R}_{t}$, and

$$
\sigma_{\alpha_{0}}:(\mathbb{R} / 2 \pi \mathbb{Z})_{\omega} \times \mathbb{R}_{E} \times \mathbb{R}_{H} \times \mathbb{R}_{t} \rightarrow \mathcal{L}_{+}^{1}\left(L^{2}(0,2 \pi)\right),
$$

is integrable with respect to $\ell_{\alpha_{0}}$, continuous with respect to $t$ and takes values in the set of nonnegative trace-class operators on $L^{2}(0,2 \pi)$. In addition, $\sigma_{\alpha_{0}}$ satisfies, for $\ell_{\alpha_{0}}$-almost every $(\omega, E, H)$ :

$$
\sigma_{\alpha_{0}}(\omega, E, H, t)=U_{\alpha_{0}, \omega}(t) \sigma_{\alpha_{0}}(\omega, E, H, 0) U_{\alpha_{0}, \omega}^{*}(t) .
$$


Finally, $\ell_{\alpha_{0}}$ and $\sigma_{\alpha_{0}}(\cdot, 0)$ only depend on the sequence of initial conditions $\left(u_{h}^{0}\right)$.

(iv) For $\alpha_{0}= \pm \frac{\pi}{2}, \nu_{\alpha_{0}}$ is a measure that does not depend on $t$, carried by $\left\{H=|\xi|^{2} / 2\right\} \cap T^{*} \partial \mathbb{D}$ and is invariant under rotations around the origin.

Remark 2.5. The conclusion of the above results (as well as their counterparts in the next section) also holds for semiclassical measures associated to sequences of approximate solutions of the Schrödinger equation, i.e. satisfying

$$
\left(D_{t}+\frac{1}{2} \Delta-V(t, z)\right) u_{h}(z, t)=o_{L_{\mathrm{loc}}^{2}(\mathbb{D} \times \mathbb{R})}(1) .
$$

Note that, as in the torus case [AM14, AFKM14], Corollary 1.1 also holds for solutions of

$$
\left(D_{t}+\frac{1}{2} \Delta\right) u_{h}(z, t)=O_{L_{\mathrm{loc}}^{2}(\mathbb{D} \times \mathbb{R})}(1),
$$

which includes for instance the case of potentials $V \in L^{\infty}\left(\mathbb{R} ; \mathcal{L}\left(L^{2}(\mathbb{D})\right)\right.$ ) (see also [Bur13] for related results).

2.5. The structure theorem: microlocal formulation. We now give the microlocal version of Theorem 2.4. The main difference is that we now use the class of test functions $\mathcal{S}_{0}$ defined in Section 2.1 .

Let $\left(u_{n}^{0}\right)$ be a sequence of initial conditions, normalized in $L^{2}(\mathbb{D})$. Denote $u_{n}(\cdot, t):=U_{V}(t) u_{n}^{0}$ and recall that $u_{n}$ also denotes the extended function by zero to whole $\mathbb{R}^{2}$. All over the paper we let $\chi \in C_{c}^{\infty}(\mathbb{R})$ be a nonnegative cut-off function that is identically equal to one near the origin. Let $R>0$. For $a \in \mathcal{S}_{0}$, we define

$$
\left\langle W_{n, R}^{\infty}, a\right\rangle:=\left\langle u_{n}, \mathrm{Op}_{1}\left(\left(1-\chi\left(\frac{|\xi|^{2}+|H|}{R^{2}}\right)\right) a(z, \xi, t, H)\right) u_{n}\right\rangle_{L^{2}\left(\mathbb{R}^{2} \times \mathbb{R}\right)},
$$

and

$$
\left\langle W_{c, n, R}, a\right\rangle:=\left\langle u_{n}, \mathrm{Op}_{1}\left(\chi\left(\frac{|\xi|^{2}+|H|}{R^{2}}\right) a(z, \xi, t, H)\right) u_{n}\right\rangle_{L^{2}\left(\mathbb{R}^{2} \times \mathbb{R}\right)} .
$$

The Calderón-Vaillancourt theorem [CV71] ensures that both $W_{n, R}^{\infty}$ and $W_{c, n, R}$ are bounded in $\mathcal{S}_{0}^{\prime}$. After possibly extracting subsequences, we have the existence of a limit: for every $a \in \mathcal{S}_{0}$,

$$
\left\langle\mu^{\infty}, a\right\rangle:=\lim _{R \rightarrow \infty} \lim _{n \rightarrow+\infty}\left\langle W_{n, R}^{\infty}, a\right\rangle,
$$

and

$$
\left\langle\mu_{c}, a\right\rangle:=\lim _{R \rightarrow \infty} \lim _{n \rightarrow+\infty}\left\langle W_{c, n, R}, a\right\rangle .
$$

As a consequence, after extraction, the subsequence $W_{n}$ converges weakly-* to a limit $\mu_{m l} \in \mathcal{S}_{0}^{\prime}$, which can be decomposed into

$$
\mu_{m l}=\mu^{\infty}+\mu_{c} .
$$

The two limit objects $\mu_{c}$ and $\mu^{\infty}$ enjoy the following first properties:

- The distribution $\mu_{c}$ vanishes if and only if the family $\left(u_{n}^{0}\right)$ converges weakly to 0 in $L^{2}(\mathbb{D})$; in other words $\mu_{c}$ reflects the "compact part" of the sequence $\left(u_{n}^{0}\right)$, hence the subscript $c$ in the notation.

- The distribution $\mu^{\infty}$ is nonnegative, 0-homogeneous and supported at infinity in the variable $(\xi, H)$ (i.e., it vanishes when paired with a compactly supported function). As a consequence, $\mu^{\infty}$ may be identified with a nonnegative Radon measure on $\mathbb{R}_{z}^{2} \times \mathbb{R}_{t} \times \mathbb{S}_{\xi, H}^{2}$. Actually, $\mu^{\infty}$ is the microlocal defect measure of [Gér91b] (with the appropriate class of symbols $\mathcal{S}_{0}$ ).

- In addition, $\mu^{\infty}$ is of the form $\mu^{\infty}(d z, d \xi, d t, d H)=\mu^{\infty}(d z, d \xi, t, d H) d t$ where $t \mapsto \mu^{\infty}(t) \in$ $L^{\infty}\left(\mathbb{R}_{t} ; \mathcal{M}_{+}\left(\mathbb{R}_{z}^{2} \times \mathbb{S}_{\xi, H}^{2}\right)\right)$. Moreover, for a.e. $t \in \mathbb{R}, \mu^{\infty}(t)$ is supported in $\left\{|\xi|^{2}=2 H\right\} \cap$ $\left(\overline{\mathbb{D}} \times \mathbb{S}_{\xi, H}^{2}\right)$. 
- The projection of the distribution $\mu_{m l}=\mu_{c}+\mu^{\infty}$ on the $(z, t)$-variables is the Radon measure $\nu$ defined in the introduction (Section 1). From the normalization of $u_{n}^{0}$ in $L^{2}$, we have for a.e. $t$ :

$$
\int_{\overline{\mathbb{D}} \times \mathbb{S}_{\xi, H}^{2}} \mu^{\infty}(d z, d \xi, t, d H) \leq 1
$$

if $u_{n}^{0} \rightarrow 0$ in $L^{2}(\mathbb{D})$, then we have $\int_{\overline{\mathbb{D}} \times \mathbb{S}_{\xi, H}^{2}} \mu^{\infty}(d z, d \xi, t, d H)=1$.

- The measure $\mu^{\infty}$ satisfies the invariance property:

$$
\left\langle\mu^{\infty}, \frac{\xi}{\sqrt{2 H}} \cdot \partial_{z} a\right\rangle=0
$$

for $a$ satisfying the symmetry condition $a(z, \xi, t, H)=a\left(z, \sigma_{z}(\xi), t, H\right)$ for $|z|=1$. In other words, $\pi_{*} \mu^{\infty}$ is invariant by the billiard flow.

These properties are well-known and won't be proven in detail here (the fact that it is carried on $\left\{H=\frac{|\xi|^{2}}{2}\right\}$ follows from Appendix $\mathrm{C}$ and the proof of invariance is essentially contained in [GL93] or [RZ09]).

Let us now discuss separately the finer properties of $\mu^{\infty}$ (high frequencies) and of $\mu_{c}$ (low frequencies).

We first describe $\mu^{\infty}$ and state the analogue of Theorem 2.4 in the microlocal setting. As previously we call $T_{(E, J)}$ the level sets of $(E, J)$ and $\mathcal{I}_{\alpha_{0}}=\left\{J=-\sin \alpha_{0} E\right\}$. The only difference with the semiclassical formalism is that the test functions are homogeneous and thus the measure $\mu^{\infty}$ is naturally defined on $\mathbb{R}_{z}^{2} \times \mathbb{R}_{t} \times \mathbb{S}_{\xi, H}^{2}$ supported by $\overline{\mathbb{D}} \times \mathbb{R}_{t} \times \mathbb{S}_{\xi, H}^{2}$. The microlocal version of Theorem 2.4 reads as follows:

Theorem 2.6. Let $\left(u_{n}^{0}\right)$ be normalized in $L^{2}(\mathbb{D})$, and such that (2.15) holds. Then the measure $\mu^{\infty}$ can be decomposed as a countable sum of nonnegative measures on $\mathbb{R}^{2} \times \mathbb{R}_{t} \times \mathbb{S}^{2}$ :

$$
\mu^{\infty}=\mu_{L e b}+\sum_{\alpha_{0} \in \pi \mathbb{Q} \cap[-\pi / 2, \pi / 2]} \mu_{\alpha_{0}},
$$

satisfying:

(i) Each of the measures in the above decomposition is carried by $\overline{\mathbb{D}} \times \mathbb{R}_{t} \times \mathbb{S}^{2} \cap\left\{|\xi|^{2}=2 H\right\}$ and by the cone $\{|J| \leq E\}$, and is invariant under the billiard flow.

(ii) The measure $\mu_{L e b}$ does not depend on $t$; $\mu_{L e b}$ is of the form $\int_{E>0,|J| \leq E} \lambda_{E, J} d \mu^{\prime}(E, J) d t$ for some nonnegative measure $\mu^{\prime}$ on $\mathbb{S}^{1}$ (i.e. the set of pairs $(E, J)$ modulo homotheties).

(iii) For every $\alpha_{0} \in \pi \mathbb{Q} \cap(-\pi / 2, \pi / 2)$, the measure $\mu_{\alpha_{0}}$ is carried by the set $\mathcal{I}_{\alpha_{0}} \cap\left\{H=E^{2} / 2\right\}$ and is defined by:

$$
\int_{\mathcal{I}_{\alpha_{0}}} a d \mu_{\alpha_{0}}=\int_{\mathcal{I}_{\alpha_{0}}} \operatorname{Tr}_{L^{2}(0,2 \pi)}\left(m_{\langle a\rangle_{\alpha_{0}}}^{\alpha_{0}} \sigma_{\alpha_{0}}\right) d \ell_{\alpha_{0}}, \quad \text { for all } a \in \mathcal{S}_{0},
$$

where $\ell_{\alpha_{0}}(d \omega, d E, d H, d t)$ is a non-negative measure on $\mathbb{R} / 2 \pi \mathbb{Z} \times\left\{E^{2}+2|H|=2\right\} \times \mathbb{R}_{t}$ carried by $\left\{H=E^{2} / 2\right\}$ and

$$
\sigma_{\alpha_{0}}:(\mathbb{R} / 2 \pi \mathbb{Z})_{\omega} \times\left\{E^{2}+2|H|=2\right\} \times \mathbb{R}_{t} \rightarrow \mathcal{L}_{+}^{1}\left(L^{2}(0,2 \pi)\right),
$$

is integrable with respect to $\ell_{\alpha_{0}}$, continuous in $t$ and takes values in the set of nonnegative trace-class operators on $L^{2}(0,2 \pi)$.

Moreover, for $\ell_{\alpha_{0}}$-almost every $(\omega, E, H)$, we have

$$
\sigma_{\alpha_{0}}(\omega, E, H, t)=U_{\alpha_{0}, \omega}(t) \sigma_{\alpha_{0}}(\omega, E, H, 0) U_{\alpha_{0}, \omega}^{*}(t) .
$$

Finally, $\ell_{\alpha_{0}}$ and $\sigma_{\alpha_{0}}(\cdot, 0)$ only depend on the sequence of initial conditions $\left(u_{n}^{0}\right)$.

(iv) For $\alpha_{0}= \pm \frac{\pi}{2}, \mu_{\alpha_{0}}$ does not depend on $t$, it is a measure carried by the set $\mathcal{I}_{ \pm \frac{\pi}{2}} \cap\{H=$ $\left.E^{2} / 2\right\}$ (which consists of vectors tangent to $\partial \mathbb{D}$ ) and is invariant under rotations around the origin.

To conclude the description of $\mu_{m l}$, it now remains to describe more precisely $\mu_{c}$. 
Theorem 2.7. There exists a nonnegative trace class operator $\rho_{0}$ on the Hilbert space $L^{2}(\mathbb{D})$ such that

$$
\left\langle\mu_{c}, a\right\rangle=\int \operatorname{Tr}_{L^{2}(\mathbb{D})}\left\{U_{V}(t)^{-1} \mathbb{1}_{\mathbb{D}} \mathrm{Op}_{1}(a(x, \xi, t, H)) \mathbb{1}_{\mathbb{D}} U_{V}(t) \rho_{0}\right\} d t
$$

(the meaning of this expression is clarified in Section 6.1).

As a consequence, the projection of $\mu_{c}$ on $\mathbb{D} \times \mathbb{R}_{t}$ is a nonnegative Radon measure, which is absolutely continuous, and continuous with respect to $t$.

Note that the ambiguity in the meaning of formula (2.18) arises when $a$ depends on $H$. If $a$ is independent of $H,(2.18)$ is the well-defined expression

$$
\left\langle\mu_{c}, a\right\rangle=\int \operatorname{Tr}_{L^{2}(\mathbb{D})}\left\{U_{V}(t)^{-1} \mathbb{1}_{\mathbb{D}} \mathrm{Op}_{1}(a(x, \xi, t)) \mathbb{1}_{\mathbb{D}} U_{V}(t) \rho_{0}\right\} d t .
$$

2.6. Link between microlocal and semiclassical Wigner measures. Let us clarify the link between the two approaches in the context of the present article (see also [Gér91a, GL93] for a related discussion).

As was said, if $\left(u_{n}^{0}\right)$ is $h_{n}$-oscillating from above and below, the semiclassical Wigner measures convey more information than the microlocal ones. In fact, if $\left(u_{n}^{0}\right)$ is $h_{n}$-oscillating from above and below (with $h_{n} \rightarrow 0$ ), we have for $a \in \mathcal{S}_{0}$

$$
\begin{aligned}
W_{u_{n}}(a)=\left\langle u_{n}, \mathrm{Op}_{1}(a(z, \xi, t, H)(\chi\right. & \left.\left.\left.\left(\frac{h_{n}^{2}\left(|\xi|^{2}+|H|\right)}{R}\right)-\chi\left(\frac{h_{n}^{2}\left(|\xi|^{2}+|H|\right)}{\epsilon}\right)\right)\right) u_{n}\right\rangle_{L^{2}\left(\mathbb{R}^{2} \times \mathbb{R}\right)} \\
& +o(1)_{\epsilon \longrightarrow 0, R \longrightarrow+\infty} \\
=\left\langle u_{n}, \mathrm{Op}_{1}\left(a_{\text {hom }}\left(z, h_{n} \xi, t, h_{n}^{2} H\right)(\chi\right.\right. & \left.\left.\left.\left(\frac{h_{n}^{2}\left(|\xi|^{2}+|H|\right)}{R}\right)-\chi\left(\frac{h_{n}^{2}\left(|\xi|^{2}+|H|\right)}{\epsilon}\right)\right)\right) u_{n}\right\rangle_{L^{2}\left(\mathbb{R}^{2} \times \mathbb{R}\right)} \\
& +o(1)_{\epsilon \longrightarrow 0, R \longrightarrow+\infty} \\
= & W_{u_{n}}^{h_{n}}\left(a_{\text {hom }}\left(\chi\left(\frac{\left(|\xi|^{2}+|H|\right)}{R}\right)-\chi\left(\frac{\left(|\xi|^{2}+|H|\right)}{\epsilon}\right)\right)\right)+o(1)_{\epsilon \longrightarrow 0, R \longrightarrow+\infty}
\end{aligned}
$$

From (2.19), one sees that if $W_{u_{n}}$ converges weakly to $\mu_{m l}$ and $W_{u_{n}}^{h_{n}}$ converges weakly to $\mu_{s c}$, and if $\left(u_{n}^{0}\right)$ is $h_{n}$-oscillating from above and below, we have

$$
\mu_{m l}(a)=\mu_{s c}\left(a_{\mathrm{hom}}\right) .
$$

The right-hand side is well-defined since $\mu_{s c}$ is a nonnegative measure which is bounded on sets of the form $\mathbb{D} \times \mathbb{R}^{2} \times[-T, T] \times \mathbb{R}($ for any $T$ ).

On the other hand, if in Theorem 2.4 the sequence $\left(u_{n}^{0}\right)$ is not $h_{n}$-oscillating from below, then $\mu_{s c}$ does charge the set $\{\xi=0\}$, and we have for any compactly supported function $a$ :

$$
\begin{aligned}
\left.\mu_{s c}\right\rceil_{(\xi, H)=0}(a) & =\lim _{\epsilon \longrightarrow 0} \lim _{\longrightarrow+\infty} W_{\chi\left(\frac{h_{n}^{2}\left(\left|D_{z}\right|^{2}+\left|D_{t}\right|\right)}{\epsilon}\right) u_{n}}(a(z, 0, t, 0)) \\
& =\lim _{\epsilon \longrightarrow 0} \lim _{\longrightarrow+\infty} W_{\chi\left(\frac{3 h_{n}^{2}\left|D_{t}\right|}{\epsilon}\right) u_{n}}(a(z, 0, t, 0)) \\
& =\lim _{\epsilon \longrightarrow 0} \lim _{\longrightarrow+\infty} W_{U_{V}(t) v_{n, \epsilon}^{0}}(a(z, 0, t, 0))
\end{aligned}
$$

where $\left.v_{n, \epsilon}^{0}=\chi\left(\frac{3 h_{n}^{2}\left|D_{t}\right|}{\epsilon}\right) u_{n}\right\rceil_{t=0}$. Equality of the first two lines comes from the fact that the measures asymptotically concentrate on $\left\{|\xi|^{2}=2 H\right\}$, and equality of the last two lines is proven in Appendix C. We see that the microlocal Wigner measures associated with $U_{V}(t) v_{n, \epsilon}^{0}$ encompass the description of $\left.\mu_{s c}\right\rceil_{(\xi, H)=0}$ : we have

$$
\left.\mu_{s c}\right\rceil_{(\xi, H)=0}(a(z, \xi, t, H))=\mu_{m l, 0}(a(z, 0, t, 0)),
$$

where $\mu_{m l, 0}$ possesses the structure described in Theorem 2.6. 
Finally, if $\left(u_{n}^{0}\right)$ is not $h_{n}$-oscillating from above, we see that

$$
\lim _{R \longrightarrow+\infty} \lim _{n \rightarrow+\infty} W_{(1-\chi)}^{h_{n}}\left(\frac{h_{n}^{2}\left(\left|D_{z}\right|^{2}+\left|D_{t}\right|\right)}{R}\right) u_{n} \quad(a)=0
$$

for compactly supported $a$, whereas the limit

$$
\lim _{R \longrightarrow+\infty} \lim _{n \rightarrow+\infty} W_{(1-\chi)}\left(\frac{h_{n}^{2}\left(\left|D_{z}\right|^{2}+\left|D_{t}\right|\right)}{R}\right) u_{n}(a)
$$

does not necessarily vanish for homogeneous $a$. This last limit coincides with

$$
\lim _{R \longrightarrow+\infty} \lim _{n \longrightarrow+\infty} W_{(1-\chi)}\left(\frac{3 h_{n}^{2}\left|D_{t}\right|}{R}\right) u_{n}(a)=\lim _{R \longrightarrow+\infty} \lim _{\longrightarrow+\infty} W_{U_{V}(t) w_{n, R}^{0}}(a)
$$

where $\left.w_{n, R}^{0}=(1-\chi)\left(\frac{3 h_{n}^{2}\left|D_{t}\right|}{R}\right) u_{n}\right\rceil_{t=0}$, and equality of the limits is proven in Appendix C. Thus, the frequencies of $u_{n}^{0}$ that are of order $\gg h_{n}^{-1}$ or $\ll h_{n}^{-1}$ are better captured by the microlocal approach.

2.7. Application to the regularity of limit measures. Theorem 2.6, applied to test functions $a \in \mathcal{S}_{0}$ that do not depend on $t$ and $H$, implies Corollary 1.1. To state a precise version of this result (say, in the semiclassical setting), we first need the following proposition.

Proposition 2.8. Suppose that $\mu_{s c}$ is a semiclassical measure associated to $\left(u_{h}\right)$ solution of (1.1)(1.2). Denote by $\bar{\mu}_{s c}(d E, d J, t)$ the image of the measure $\mu_{s c}(d z, d \xi, t, d H)$ under the moment map

$$
M:(z=(x, y), \xi, H) \mapsto(E, J)=\left(|\xi|, x \xi_{y}-y \xi_{x}\right)
$$

(velocity and angular momentum). Then $\bar{\mu}_{s c}$ does not depend on $t$.

This proposition is proved in Section 5.1. Arguments developed in [AM14] (and that we do not reproduce here) show that Corollary 1.1 can be refined as follows.

Theorem 2.9. Define by $\mu_{E, J}(t, \cdot)$ is the disintegration of $\mu_{s c}(t, \cdot)$ with respect to the variables $(E, J)$, carried on the 2-dimensional (lagrangian) manifold $T_{(E, J)}=\left\{(z, \xi),\left(|\xi|, x \xi_{y}-y \xi_{x}\right)=\right.$ $(E, J)\}$, i.e.

$$
\begin{aligned}
\int_{\mathbb{R}_{H}} \int_{\overline{\mathbb{D}} \times \mathbb{R}^{2}} f(z, \xi, t, H) \mu_{s c}(d z, d \xi, t, d H) & \\
& =\int_{\mathbb{R}^{2}}\left(\int_{T_{(E, J)}} f\left(z, \xi, t, \frac{E^{2}}{2}\right) \mu_{E, J}(t, d z, d \xi)\right) \bar{\mu}_{s c}(d E, d J),
\end{aligned}
$$

for every bounded measurable function $f$, for $t \in \mathbb{R}$.

Then for $\bar{\mu}_{s c}$-almost every $(E, J)$ with $|J| \neq E$, the measure $\mu_{E, J}(t, \cdot)$ is absolutely continuous on $T_{(E, J)}$.

Note that $|J|=E$, with $E \neq 0$, means that $T_{(E, J)} \cap\left(\overline{\mathbb{D}} \times \mathbb{R}^{2}\right)$ is contained in the set $\{(z, \xi),|z|=$ $1, z \perp \xi\}$ of tangent rays to the boundary. The restriction of $\mu_{s c}(t)$ to that set may be considered trivial, since (2.11) implies that it is invariant under rotation.

Finally, for $J=E=0$, we can use the last lines of Section 2.6, combined with Theorem 2.6: the measure $\mu_{s c}$ restricted to $\{\xi=0\}=\overline{\mathbb{D}} \times\{0\}$ is the sum of an absolutely continuous measure carried by the interior $\mathbb{D}$ and a multiple of the Lebesgue measure on $\partial \mathbb{D}$.

Remark 2.10. The analogues of Proposition 2.8 and Theorem 2.9 hold as well in the microlocal setting. In particular, if $\bar{\mu}^{\infty}$ is the image of $\mu^{\infty}(t)$ under the map $(z, \xi, t, H) \mapsto(E, J)$, this measure is independent of $t$.

Remark 2.11. Proposition 2.8 (and Remark 2.10) allows us to complete the proof of the necessity of the assumption $\Omega \cap \partial \mathbb{D} \neq \emptyset$ in Theorem 1.2 when $V$ does not identically vanish (see the discussion in Section 1.6.2). Taking for instance as initial data $u_{n}^{0}:=\psi_{n, 0}^{ \pm} /\left\|\psi_{n, 0}^{ \pm}\right\|_{L^{2}(\mathbb{D})}$ (see (1.7)) with and $n \rightarrow \infty$, then one has $\left|u_{n}^{0}\right|^{2} d x \rightarrow(2 \pi)^{-1} \delta_{\partial \mathbb{D}}$ (see Section 1.3); more precisely, the Wigner measures associated with the initial data $u_{n}^{0}:=\psi_{n, 0}^{ \pm} /\left\|\psi_{n, 0}^{ \pm}\right\|_{L^{2}(\mathbb{D})}$ concentrate on the set $\{|J|=E\}$. 
Combined with Proposition 2.8 (and Remark 2.10), this shows that $\bar{\mu}^{m l}$ is entirely carried by the set $\{|J|=E\}$, and thus $\mu^{m l}$ itself does not charge the interior of the disk, where $|J|<E$. This shows that (1.5) cannot hold if $\Omega$ does not touch the boundary.

2.8. Measures at the boundary. In this section, we define and compare different measures on $\partial \mathbb{D}$. Given an invariant measure for the billiard flow, we first define the associated "projected measure" on the boundary. Second, we define semiclassical and microlocal measures associated with the Neumann trace at the boundary of sequences of solutions of (1.1). We finally explain the links between these three objects.

2.8.1. Projection on the boundary of an invariant measure. We observe the following standard construction from the theory of Poincaré sections in dynamical systems. Let $S=\{(z, \xi),|z|=$ $1, \xi \cdot z \neq 0\}$, union of $S^{+}=\{(z, \xi),|z|=1, \xi \cdot z>0\}$ (vectors pointing outwards) and of $S^{-}=$ $\{(z, \xi),|z|=1, \xi \cdot z<0\}$ (vectors pointing inwards). When $(z, \xi) \in S^{+}$, we denote as above by $\alpha(z, \xi)=-\arcsin \left(\frac{J(z, \xi)}{|\xi|}\right)$ the angle of the vector $\xi$ with the normal at $z$ to the disk. The map

$$
\begin{aligned}
P:\left\{(z, \xi, \tau) \in S^{+} \times \mathbb{R}, \tau \in[0,2 \cos \alpha(z, \xi)]\right\} & \longrightarrow \overline{\mathbb{D}} \times \mathbb{R}^{2} \\
(z, \xi, \tau) & \mapsto\left(z+\frac{\tau}{|\xi|} \sigma_{z}(\xi), \sigma_{z}(\xi)\right)
\end{aligned}
$$

is a measurable bijection onto its image $S \cup\left(\mathbb{D} \times \mathbb{R}^{2}\right)$, and $\pi \circ P$ is a measurable bijection onto its image (recall that $\pi$ is the projection from $\overline{\mathbb{D}} \times \mathbb{R}^{2}$ to $\mathbb{W}$ ). If $\mu$ is a nonnegative measure on $S \cup\left(\mathbb{D} \times \mathbb{R}^{2}\right)$ which does not charge $S$, and such that $\pi_{*} \mu$ is invariant under the billiard flow, then $P_{*}^{-1} \mu$ must be of the form

$$
P_{*}^{-1} \mu=\mu^{S} \otimes d \tau
$$

where $\mu^{S}$ is a measure on $S^{+}$which is invariant under the first return map

$$
(z, \xi) \mapsto\left(z+\frac{2 \cos \alpha(z, \xi)}{|\xi|} \sigma_{z}(\xi), \sigma_{z}(\xi)\right)
$$

This implies that

$$
\begin{aligned}
\int_{\overline{\mathbb{D}} \times \mathbb{R}^{2}} \xi . \partial_{z} a d \mu & =\int|\xi| \partial_{\tau}(a \circ P) d \mu^{S} \otimes d \tau \\
& =\int_{S^{+}}|\xi|\left(a\left(z+\frac{2 \cos \alpha}{|\xi|} \sigma_{z}(\xi), \sigma_{z}(\xi)\right)-a\left(z, \sigma_{z}(\xi)\right)\right) \mu^{S}(d z, d \xi) \\
& =\int_{S^{+}}|\xi|\left(a(z, \xi)-a\left(z, \sigma_{z}(\xi)\right)\right) \mu^{S}(d z, d \xi) .
\end{aligned}
$$

Note that the total mass of $\mu$ is $\int d \mu=\int_{S^{+}} 2 \cos \alpha(z, \xi) d \mu^{S}(z, \xi)$.

2.8.2. Semiclassical measure associated to Neumann trace. Let $\left(u_{h}^{0}\right)$ be a family of initial conditions, normalized in $L^{2}(\mathbb{D})$. When we look at the semiclassical Wigner distributions (2.4), where we use compactly supported symbols, Remarks 2.3 and C.3 show that we may truncate $\left(u_{h}^{0}\right)$ in frequency and assume, without changing the limit as $h \longrightarrow 0$, that $u_{h}^{0} \in H_{0}^{1}(\mathbb{D}),\left\|\nabla u_{h}^{0}\right\|_{L^{2}(\mathbb{D})}=$ $O\left(h^{-1}\right)$. Proposition C.1 then entails that the boundary data $h \partial_{n}\left(U_{V}(t) u_{h}^{0}\right)$ form a bounded sequence in $L_{\text {loc }}^{2}(\mathbb{R} \times \partial \mathbb{D})$.

Now, let $\mu_{s c}^{\partial} \in \mathcal{M}_{+}\left(T^{*} \partial \mathbb{D} \times T^{*} \mathbb{R}\right)$ be a semiclassical measure associated with the boundary data $h \partial_{n} u_{h}(t)$ defined by quantizing test functions on $T^{*} \partial \mathbb{D} \times T^{*} \mathbb{R}$ with the same scaling $\left(h j, h^{2} H\right)$ in the cotangent variables as in (2.7). Then $\mu_{s c}^{\partial}$ is carried by the set $\left\{(u, j, t, H) \in T^{*} \partial \mathbb{D} \times T^{*} \mathbb{R},|j| \leq\right.$ $\sqrt{2 H}\}$. If $\mu_{s c}$ and $\mu_{s c}^{\partial}$ are obtained through the same sequence of initial data, then we have the relation (see [GL93])

$$
\int_{\overline{\mathbb{D}} \times \mathbb{R}^{2} \times \mathbb{R} \times \mathbb{R}} \xi \cdot \partial_{z} a \mu_{s c}(d z, d \xi, t, d H) d t=\int \frac{a\left(u, \xi^{+}(j, H)\right)-a\left(u, \xi^{-}(j, H)\right)}{2 \sqrt{2 H-j^{2}}} \mu_{s c}^{\partial}(d u, d j, d t, d H),
$$


valid for any smooth function $a$. For $(u, j) \in T^{*} \partial \mathbb{D}$ with $|j| \leq \sqrt{2 H}$, the vectors $\xi^{ \pm}(j, H)$ are the two vectors (pointing outwards and inwards) in $T_{u}^{*} \mathbb{R}^{2}$ of norm $=\sqrt{2 H}$, whose projection to $T_{u}^{*} \partial \mathbb{D}$ is $j$. Note that the expression under the integral on the right hand side of (2.22) has a well-defined finite limit as $|j| \longrightarrow \sqrt{2 H}$. Identity (2.22) has three consequences:

- First, the measure $\mu_{s c}$ does not charge the set $S$ defined in Section 2.2 (otherwise the left-hand side of (2.22) would define a distribution of order 1 which is not a measure). Note that (2.22) is stronger than (2.11).

- Second, let $\mu_{s c}^{S}(t)$ be the measure associated to $\mu_{s c}(t)$ as in Section 2.8.1. Comparing (2.22) with (2.21), we see that for any $a$ defined on $S^{+}$,

$$
\begin{aligned}
\int_{(u, j) \in T^{*} \partial \mathbb{D},|j|<\sqrt{2 H}} a\left(u, \xi^{+}(j, H), t, H\right) \mu_{s c}^{\partial}(d u & d j, d t, d H) \\
& =\int_{S^{+}} 2|\xi|^{2} \cos \alpha(z, \xi) a(z, \xi) \mu_{s c}^{S}(d z, d \xi, t, d H) d t .
\end{aligned}
$$

- Third, (2.22) implies

$$
\int_{T^{*} \partial \mathbb{D} \times \mathbb{R} \times \mathbb{R}}|\xi|^{2} a(z, \xi, t, H) \mu_{s c}(d z, d \xi, t, d H) d t=\int_{|j|=\sqrt{2 H}} a(u, j, t, H) \mu_{s c}^{\partial}(d u, d j, d t, d H) .
$$

In particular, note that $\left.\mu_{s c}^{\partial}\right\rceil_{H=0}$ vanishes, since $H=0$ corresponds to $\xi=0$ on the left-hand side.

Identities (2.11) and (2.22) are essentially proven in [GL93] (see also [RZ09]) for general domains (for time-independent solutions of (1.1)); we do not reproduce the proofs here.

2.8.3. Microlocal measure associated to Neumann trace. The sequences considered here $u_{n}=$ $U_{V}(t) u_{n}^{0}$ are bounded in $L^{\infty}\left(\mathbb{R} ; L^{2}(\mathbb{D})\right)$. Since normal traces are not convenient to work with at this level of regularity, the definition of associated microlocal measures needs a little care.

For this, let us first define $\psi \in C^{\infty}(\mathbb{R})$, such that $\psi=0$ on $(-\infty, 1]$ and $\psi=1$ on $[2,+\infty)$ and the operator $A\left(D_{t}\right)=\mathrm{Op}_{1}\left(\frac{\psi(H)}{\sqrt{2 H}}\right)$. We have the following regularity result.

Lemma 2.12. For all $\varphi \in C^{\infty}\left(\mathbb{R}_{t} \times \overline{\mathbb{D}}_{z}\right)$ with compact support in the first variable $t \in \mathbb{R}$, there exists a constant $C=C(\varphi, \psi)>0$ such that for any $u^{0} \in L^{2}(\mathbb{D})$, the associated solution $u(t)=$ $U_{V}(t) u^{0}$ satisfies

$$
\left\|A\left(D_{t}\right) \varphi u\right\|_{L^{2}\left(\mathbb{R} ; H^{1}(\mathbb{D})\right)} \leq C\left\|u^{0}\right\|_{L^{2}(\mathbb{D})} .
$$

This Lemma is proved at the end of Appendix C. We now define, for any $g \in C_{c}^{\infty}(\mathbb{R})$, the sequence $\tilde{u}_{n}=A\left(D_{t}\right) g(t) u_{n}$, solution of

$$
\left(D_{t}+\frac{1}{2} \Delta\right) \tilde{u}_{n}=A\left(D_{t}\right)\left(g(t) V(t, z)+i g^{\prime}(t)\right) u_{n}
$$

As a consequence of Lemma 2.12, we have $\left\|\tilde{u}_{n}\right\|_{L^{2}\left(\mathbb{R} ; H^{1}(\mathbb{D})\right)} \leq C\left\|u_{n}^{0}\right\|_{L^{2}(\mathbb{D})}$ together with

$$
\left\|A\left(D_{t}\right)\left(g(t) V(t, z) u_{n}+i g^{\prime}(t)\right) u_{n}\right\|_{L^{2}\left(\mathbb{R} ; H^{1}(\mathbb{D})\right)} \leq C\left\|u_{n}^{0}\right\|_{L^{2}(\mathbb{D})} .
$$

Equation (2.23) then implies that $\left\|\tilde{u}_{n}\right\|_{L^{\infty}\left(\mathbb{R} ; H^{1}(\mathbb{D})\right)} \leq C\left\|u_{n}^{0}\right\|_{L^{2}(\mathbb{D})}$ and that $A\left(D_{t}\right) g(t) \partial_{n} u_{n}=\partial_{n} \tilde{u}_{n}$ is bounded in $L^{2}(\mathbb{R} \times \partial \mathbb{D})$ by $\left\|u_{n}^{0}\right\|_{L^{2}(\mathbb{D})}$, according to the hidden regularity of Proposition C.1. Hence, if we take $g$ to be constant equal to 1 on the support of $a$, after extraction of subsequences, the following limit exists

$$
\left\langle\mu_{m l}^{\partial}, a\right\rangle=\lim _{R \rightarrow \infty} \lim _{n \rightarrow+\infty}\left\langle\partial_{n} \tilde{u}_{n}, \mathrm{Op}_{1}\left(\left(1-\chi\left(\frac{|H|}{R^{2}}\right)\right) a(u, j, t, H)\right) \partial_{n} \tilde{u}_{n}\right\rangle_{L^{2}(\partial \mathbb{D} \times \mathbb{R})},
$$

for symbols $a \in C^{\infty}\left(T^{*}(\partial \mathbb{D} \times \mathbb{R})\right)$, compactly supported in the variables $z, t$, such that

$$
a(u, j, t, H)=a\left(u, \lambda j, t, \lambda^{2} H\right), \quad \text { for }|H|>R_{0} \text { and } \lambda \geq 1 .
$$


Then $\mu_{m l}^{\partial}$ is carried by the set $\left\{(u, j, t, H) \in T^{*} \partial \mathbb{D} \times T^{*} \mathbb{R},|j| \leq \sqrt{2 H}\right\}$. If moreover $\mu_{m l}$ and $\mu_{m l}^{\partial}$ are obtained through the same sequence of initial data, then we have the relation (see again [GL93])

$$
\begin{aligned}
\int_{\overline{\mathbb{D}} \times \mathbb{R}_{t} \times \mathbb{S}_{\xi, H}^{2}} \frac{\xi}{\sqrt{2 H}} \cdot \partial_{z} a \mu^{\infty}(d z, d \xi, t, d H) d t \\
=\int_{|j| \leq \sqrt{2 H}} \frac{1}{2}\left(\frac{2 H}{2 H-j^{2}}\right)^{\frac{1}{2}}\left(a\left(u, \xi^{+}(j, H)\right)-a\left(u, \xi^{-}(j, H)\right)\right) \mu_{m l}^{\partial}(d u, d j, d t, d H)
\end{aligned}
$$

valid for any $a \in \mathcal{S}_{0}$. The vectors $\xi^{ \pm}(j, H)$ are the two vectors (pointing outwards and inwards) in $T_{u}^{*} \mathbb{R}^{2}$ of norm $=\sqrt{2 H}$, whose projection to $T_{u}^{*} \partial \mathbb{D}$ is $j$. As above, this implies that $\mu^{\infty} \operatorname{does}$ not charge the set $S$; we then denote by $\mu_{m l}^{S}(t)$ the measure associated to $\mu^{\infty}(t)$ as in Section 2.8.1. Comparing with (2.21), we see that for any $a \in \mathcal{S}_{0}$, we have

$$
\begin{aligned}
\int_{(u, j) \in T^{*} \partial \mathbb{D},|j|<\sqrt{2 H}} a\left(u, \xi^{+}(j, H), t, H\right) \mu_{m l}^{\partial} & (d u, d j, d t, d H) \\
& =\int_{S^{+}} 2 \cos \alpha(z, \xi) a(z, \xi) \mu_{m l}^{S}(d z, d \xi, t, d H) d t .
\end{aligned}
$$

Moreover, (2.24) implies

$$
\begin{aligned}
\int_{(u, \xi) \in T^{*} \partial \mathbb{D},|\xi|=\sqrt{2 H}} a(z, \xi, t, H) \mu^{\infty}(d z, d \xi, t, d H) d t & \\
= & \int_{|j|=\sqrt{2 H}} a(u, j, t, H) \mu_{m l}^{\partial}(d u, d j, d t, d H) .
\end{aligned}
$$

These links between the different measures shall be in particular useful when proving the boundary observability result of Theorem 1.3.

2.9. Plan of the proofs. Section 3 first deals with the understanding of action-angle coordinates and the appropriate decomposition of measures that are invariant by the billiard flow. Section 3.1 discusses in more detail the coordinates described in the introduction, in which the dynamics of the billiard can be integrated and introduces the Fourier Integral Operator corresponding to this change of coordinates. Section 3.2 reduces the study of invariant measures on the disk to their restriction to all invariant tori of the dynamics (more precisely, their restriction to the level sets $\mathcal{I}_{\alpha}$, which are unions of invariant tori)

Sections 4 and 5 are devoted to the proof of Theorem 2.4 (semiclassical version of the result). In Section 4 , we perform the second microlocalization on a level set $\mathcal{I}_{\alpha}$ : we start by introducing the adapted class of symbols in Section 4.1 and the appropriate coordinates (which are a modification of the action-angle coordinates) in Section 4.2. This allows us to construct the two different second-microlocal measures in Section 4.3. We then prove their structure properties in Sections 4.4 and 4.5. To complete the analysis, we prove that they obey invariance laws in Section 4.4 and 4.6 respectively. Section 5 then concludes the proof of Theorem 2.4.

In Section 6 we explain how to adapt the proof to obtain the microlocal version, Theorem 2.6.

The observability inequalities of Theorems 1.2 and 1.3 are then derived in Section 7 .

Appendices A and B are devoted to the technical calculations needed to change coordinates from polar to action-angle ones. Appendix C is a technical elaboration on the "hidden regularities" of solutions of Schrödinger equations. Finally, Appendix D states and proves the $L^{\infty}$ regularity in time of Wigner measures associated to solutions of the Schrödinger equation.

\section{ACTION-ANGLE COORDINATES AND DECOMPOSITION OF INVARIANT MEASURES}

3.1. Action-angle coordinates and their quantization. Recall that the change of coordinates $\Phi$, mapping action-angle coordinates to cartesian ones, is introduced in Section 2.2 (see (2.5)). The map

$$
\Phi:\{(s, \theta, E, J): E>0, \theta \in \mathbb{R} / 2 \pi \mathbb{Z}, s \in \mathbb{R}, J \in \mathbb{R}\} \longrightarrow\left\{(z, \xi) \in \mathbb{R}^{2} \times \mathbb{R}^{2}: \xi \neq 0\right\}
$$


is a diffeomorphism satisfying, in particular,

$$
\Phi^{-1}\left(\mathbb{D} \times\left(\mathbb{R}^{2} \backslash\{0\}\right)\right)=\left\{(s, \theta, E, J):(\theta, E) \in \mathbb{R} / 2 \pi \mathbb{Z} \times(0, \infty),(J / E)^{2}+s^{2}<1\right\} .
$$

Note that the hamiltonian flow of the energy $\frac{E^{2}}{2}$ (the geodesic flow) reads

$$
G^{\tau}:(s, \theta, E, J) \mapsto(s+\tau E, \theta, J, E), \quad \tau \in \mathbb{R},
$$

and the hamiltonian flow of $J$ (unit speed rotation) is given by:

$$
R^{\tau}:(s, \theta, E, J) \mapsto(s, \theta+\tau, J, E), \quad \tau \in \mathbb{R} .
$$

Write for $\theta \in \mathbb{R} / 2 \pi \mathbb{Z}$,

$$
\omega(\theta):=(-\sin \theta, \cos \theta)
$$

the transformation $\Phi$ admits the generating function

$$
S(z, \theta, s, E)=E \omega(\theta) \cdot z-E s,
$$

meaning that

$$
\begin{aligned}
\operatorname{Graph} \Phi & =\{(s, \theta, E, J, z, \xi):(z, \xi)=\Phi(s, \theta, E, J)\} \\
& =\left\{(s, \theta, E, J, z, \xi): \frac{\partial S}{\partial E}=0, \xi=\frac{\partial S}{\partial z}, J=-\frac{\partial S}{\partial \theta}, E=-\frac{\partial S}{\partial s}\right\} .
\end{aligned}
$$

The existence of such a generating function implies that the diffeomorphism $\Phi$ preserves the symplectic form (see for instance [Zwo12, Theorem 2.7]), i.e.

$$
d \xi_{x} \wedge d x+d \xi_{y} \wedge d y=d E \wedge d s+d J \wedge d \theta
$$

Using this generating function we define a unitary operator that quantises the canonical transformation $\Phi$. The operator

$$
\mathscr{U} f(s, \theta)=(2 \pi h)^{-3 / 2} \int_{0}^{\infty} \int_{\mathbb{R}^{2}} e^{-i \frac{S(z, \theta, s, E)}{h}} f(z) \sqrt{E} d z d E,
$$

mapping functions on $\mathbb{R}_{z}^{2}$ to functions on $\mathbb{R}_{s} \times \mathbb{R}_{\theta}$ is in fact a semiclassical Fourier Integral Operator associated with $\Phi$ (the choice of the term $\sqrt{E}$ in this expression is explain by Lemma 3.1 below). Note that $\mathscr{U} f$ can be also written independently of $h$ as:

$$
\mathscr{U} f(s, \theta)=\int_{0}^{\infty} e^{i E s} \widehat{f}(E \omega(\theta)) \sqrt{E} \frac{d E}{(2 \pi)^{3 / 2}}
$$

where $\widehat{f}$ stands for the Fourier transform of $f$. Therefore, the Fourier transform with respect to $s$ of $\mathscr{U} f(s, \theta)$ is merely:

$$
(2 \pi)^{-1 / 2} \widehat{f}(E \omega(\theta)) \mathbb{1}_{[0, \infty)}(E) \sqrt{E} .
$$

From this it is clear that for any symbol $\phi: \mathbb{R} \rightarrow \mathbb{R}$ one has:

$$
\phi\left(D_{s}\right) \mathscr{U} f=\mathscr{U} \phi\left(\left|D_{z}\right|\right) f,
$$

and, by Placherel's theorem,

$$
\begin{aligned}
\int_{0}^{2 \pi} \int_{0}^{\infty} \mathscr{U} f(s, \theta) \overline{\mathscr{U} g(s, \theta)} d s d \theta & =\int_{0}^{2 \pi} \int_{0}^{\infty} \widehat{f}(E \omega(\theta)) \overline{\widehat{g}(E \omega(\theta))} E \frac{d s d \theta}{(2 \pi)^{2}} \\
& =\langle g, f\rangle_{L^{2}\left(\mathbb{R}^{2}\right)}
\end{aligned}
$$

In particular, the following Lemma has been proved:

Lemma 3.1. (i) The operator $\mathscr{U}$ is unitary from $L^{2}\left(\mathbb{R}^{2}\right)$ to $L^{2}(\mathbb{R} \times \mathbb{R} / 2 \pi \mathbb{Z})$ : $\mathscr{U}^{*} \mathscr{U}=I$.

(ii) For $f \in C_{c}^{\infty}\left(\mathbb{R}^{2}\right)$, we have $\partial_{s}^{2} \mathscr{U} f=\mathscr{U} \Delta f$.

As a consequence,

$$
-h^{2} \mathscr{U} \Delta \mathscr{U}^{*}=-h^{2} \partial_{s}^{2}
$$


Notation. We denote by $P_{0}(z, \xi)=\frac{|\xi|^{2}}{2}$ the hamiltonian generating the geodesic flow in $\mathbb{R}^{2} \times \mathbb{R}^{2}$; and $P_{1}(z, \xi)=x \xi_{y}-y \xi_{x}$ the hamiltonian generating the (unit speed) rotation. We denote by $X_{P_{0}}=\xi \cdot \partial_{z}$ and $X_{P_{1}}=z^{\perp} \cdot \partial_{z}+\xi^{\perp} \cdot \partial_{\xi}$ the corresponding hamiltonian vector fields on $T^{*} \mathbb{R}^{2}$. We denote by $G^{\tau}(z, \xi)=(z+\tau \xi, \xi)$ the geodesic flow (generated by $P_{0}$ ) and $R^{\tau}$ the flow generated by $P_{1}$ (rotation of angle $\tau$ of both $z$ and $\xi$ ).

In the new coordinates, these hamiltonians and vector fields are slightly simpler since $P_{0} \circ \Phi=$ $\frac{E^{2}}{2}, P_{1} \circ \Phi=J$ together with $X_{P_{0} \circ \Phi}=E \partial_{s}$ and $X_{P_{1} \circ \Phi}=\partial_{\theta}$. Very often, we shall (with a slight abuse of notation) use the letter $J$ to mean the function $P_{1}$, and $E$ for the function $\sqrt{2 P_{0}}$.

3.2. Decomposition of an invariant measure of the billiard . This section aims at describing properties shared by all measures $\mu$ invariant by the billiard flow (even if they are not necessarily linked with solutions to a partial differential equation). It essentially collects a few simple facts that will be useful in the next sections when studying measures arising from solutions of the Schrödinger equation (1.1).

Let $(z, \xi) \in \overline{\mathbb{D}} \times \mathbb{R}^{2}$. There exist $t_{1} \leq 0, t_{2} \geq 0$ such that $\left|z+t_{1} \xi\right|=\left|z+t_{2} \xi\right|=1$. Note that if $(z, \xi) \in \mathbb{D} \times \mathbb{R}^{2}$, then $t_{1}$ and $t_{2}$ are unique and $t_{1}>0, t_{2}<0$.

Recall that $\alpha \in[-\pi / 2, \pi / 2]$ (defined in Section 2.2) is the oriented angle between $-\left(z+t_{1} \xi\right.$ ) and $\xi$ (that is, the angle between the velocity $\xi$ and the inner normal to the disk, at the point where the oriented straight line $\{z+t \xi, t \in \mathbb{R}\}$ first hits the disk). See Figure 2. One has the expression

$$
\alpha=-\arcsin \left(\frac{x \xi_{y}-y \xi_{x}}{|\xi|}\right) .
$$

Our work is based on the following partition of phase space:

$$
\overline{\mathbb{D}} \times\left(\mathbb{R}^{2} \backslash\{0\}\right)=\alpha^{-1}(\pi \mathbb{Q} \cap[-\pi / 2, \pi / 2]) \sqcup \alpha^{-1}(\mathbb{R} \backslash \pi \mathbb{Q}),
$$

from which the following lemma follows.

Lemma 3.2. Let $\mu$ be any finite, nonnegative Radon measure ${ }^{1}$ on $\overline{\mathbb{D}} \times \mathbb{R}^{2}$. Then $\mu$ decomposes as a sum of nonnegative measures:

$$
\left.\left.\mu=\mu\rceil_{\alpha \notin \pi \mathbb{Q}}+\sum_{r \in \mathbb{Q} \cap[-1 / 2,1 / 2]} \mu\right\rceil_{\alpha=r \pi}+\mu\right\rceil_{\xi=0} .
$$

Note that the functions $P_{0}, P_{1}$, and thus also $\alpha$, are well-defined on the billiard phase space $\mathbb{W}$. Thus the previous lemma applies as well to measures on $\mathbb{W}$.

In what follows, we shall call nonnegative invariant measure a nonnegative Radon measure on $\mathbb{W}$ which is invariant under the billiard flow. We shall extend this terminology to measures $\mu$ defined a priori on $\overline{\mathbb{D}} \times \mathbb{R}^{2}$, to mean that $\pi_{*} \mu$ (the image of $\mu$ under the projection $\pi$ ) is invariant under the billiard flow $\phi^{\tau}$ on $\mathbb{W}$.

Lemma 3.3. Let $\mu$ be a nonnegative invariant measure on $\mathbb{W}$. Then every term in the decomposition (3.2) is a nonnegative invariant measure, and $\mu\rceil_{\alpha \notin \pi \mathbb{Q}}$ is invariant under the rotation flow $\left(R^{\tau}\right)$, as well as $\left.\mu\right\rceil_{\alpha= \pm \pi / 2}$.

The rotation flow $\left(R^{\tau}\right)$ is well defined on $\mathbb{W}$, so the last sentence makes sense. The assertion for $\alpha= \pm \pi / 2$ comes from the fact that the rotation flow coincides with the billiard flow (up to time change) on the set $\{\alpha= \pm \pi / 2\}$. The assertion for $\alpha \notin \pi \mathbb{Q}$ is a standard fact. It comes from the remark that, for any given value $\alpha_{0}$ (such that $\alpha_{0} \notin \pi \mathbb{Q}$ ) we can find $T=T\left(\alpha_{0}\right)>0$ such that $\phi^{T}$ coincides with an irrational rotation on the set $\left\{\alpha=\alpha_{0}\right\}$.

Thus, for $\alpha \notin \pi \mathbb{Q}$ or $\alpha= \pm \pi / 2$, there is nothing to prove to get Theorem 2.9.

Now consider a term $\mu\rceil_{\alpha=r_{0} \pi}$, where $r_{0} \in \mathbb{Q} \cap(-1 / 2,1 / 2)$ is fixed. Let us denote $\alpha_{0}=\pi r_{0}$. Introduce the vector field on $T^{*} \mathbb{R}^{2}$ :

$$
\left(\alpha_{0}-\alpha\right) X_{P_{1}}+\frac{\cos \alpha}{E} X_{P_{0}}
$$

\footnotetext{
${ }^{1}$ We denote by $\mathcal{M}_{+}\left(\overline{\mathbb{D}} \times \mathbb{R}^{2}\right)$ the set of all such measures.
} 
On the set $\mathcal{I}_{\alpha_{0}}=\left\{J=-\sin \alpha_{0} E\right\}$ it coincides with $X_{P_{0}}$ up to a constant factor. Denote by $\phi_{\alpha_{0}}^{\tau}$ the flow on $\mathbb{W}$ generated by $\left(\alpha_{0}-\alpha\right) X_{P_{1}}+\frac{\cos \alpha}{E} X_{P_{0}}$ with reflection on the boundary of the disk. More precisely, it is the unique continuous flow defined on $\mathbb{W}$ such that

$$
\phi_{\alpha_{0}}^{\tau}(z, \xi)=R^{\left(\alpha_{0}-\alpha\right) \tau}\left(z+\tau \frac{\cos \alpha}{|\xi|} \xi, \xi\right)
$$

whenever $z \in \mathbb{D}$ and $z+\tau \frac{\cos \alpha}{|\xi|} \xi \in \mathbb{D}$ (with $\alpha=-\arcsin \frac{P_{1}(z, \xi)}{|\xi|}$ as previously).

In the coordinates $(s, \theta, E, J)$, this flow simply reads

$$
\phi_{\alpha_{0}}^{\tau} \circ \Phi(s, \theta, E, J)=\Phi\left(s+\tau \cos \alpha, \theta+\left(\alpha_{0}-\alpha\right) \tau, E, J\right), \quad \alpha=-\arcsin (J / E),
$$

with reflection on the boundary of the disk.

All its orbits are periodic: actually, we determined the coefficients $\left(\alpha_{0}-\alpha\right)$ and $\frac{\cos \alpha}{E}$ precisely for that purpose, see Figure 3. Some trajectories of the flow are represented on Figure 4.

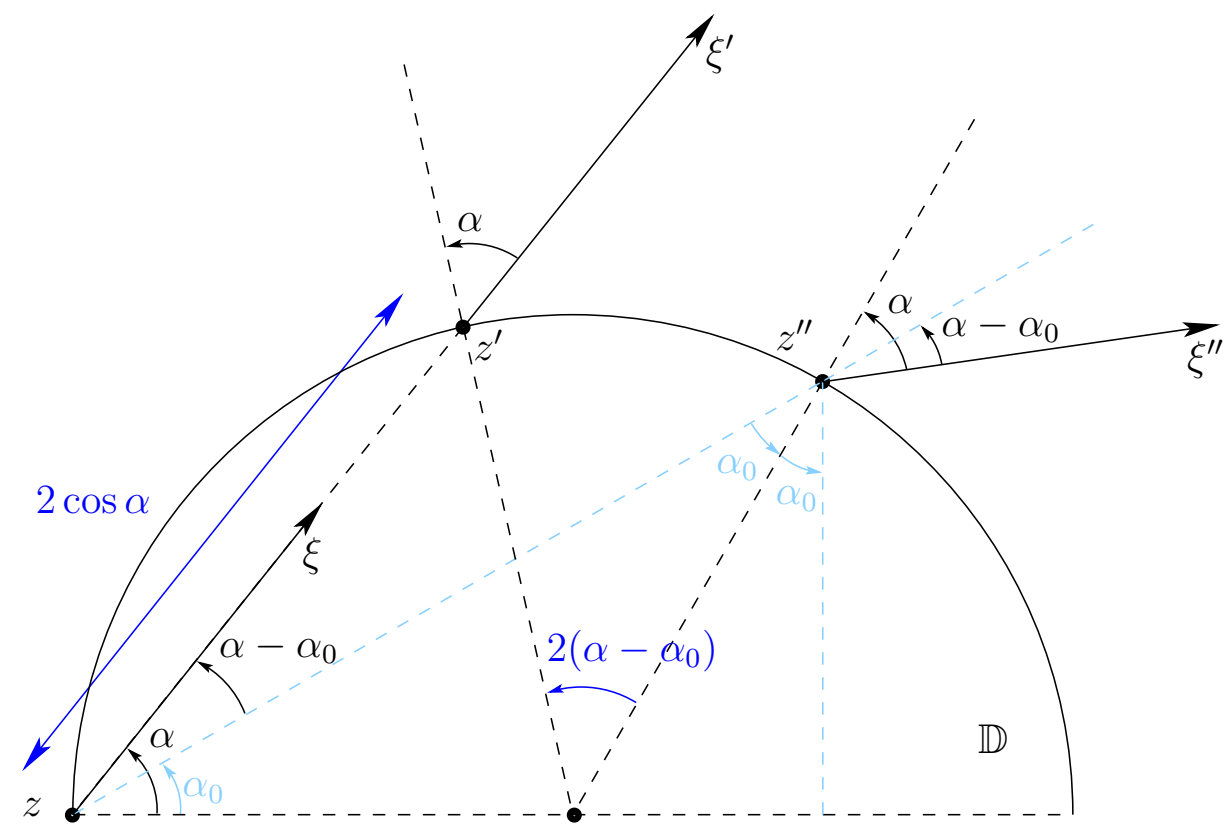

Figure 3. Construction of the flow $\phi_{\alpha_{0}}^{\tau}$ with $\alpha_{0}=\pi / 6$.

On the figure, $\left(z^{\prime}, \xi^{\prime}\right)=\left(z+2 \frac{\cos \alpha}{|\xi|} \xi, \xi\right)$ and $\left(z^{\prime \prime}, \xi^{\prime \prime}\right)=R^{2\left(\alpha_{0}-\alpha\right)}\left(z^{\prime}, \xi^{\prime}\right)=\phi_{\alpha_{0}}^{2}(z, \xi)$.

The following lemma is now obvious.

Lemma 3.4. Let $\mu$ be a nonnegative invariant measure on $\mathbb{W}$.

Let $a \in C^{0}(\mathbb{W})$. Then

$$
\left.\left.\int a \circ \phi_{\alpha_{0}}^{\tau} d \mu\right\rceil_{\alpha=\alpha_{0}}=\int a d \mu\right\rceil_{\alpha=\alpha_{0}}
$$

for every $t \in \mathbb{R}$.

Equivalently, we have

$$
\left.\left.\int a d \mu\right\rceil_{\alpha=\alpha_{0}}=\int\langle a\rangle_{\alpha_{0}} d \mu\right\rceil_{\alpha=\alpha_{0}}
$$

where

$$
\langle a\rangle_{\alpha_{0}}=\lim _{T \longrightarrow+\infty} \frac{1}{T} \int_{0}^{T} a \circ \phi_{\alpha_{0}}^{\tau} d t .
$$

Remark that $\langle a\rangle_{\alpha_{0}}$ is well defined even if $a$ is a bounded measurable function on $\mathbb{D}$ (the times $\tau$ where the trajectories of $\phi_{\alpha_{0}}^{\tau}$ hit the boundary form a set of measure 0 in $\mathbb{R}$ ). 


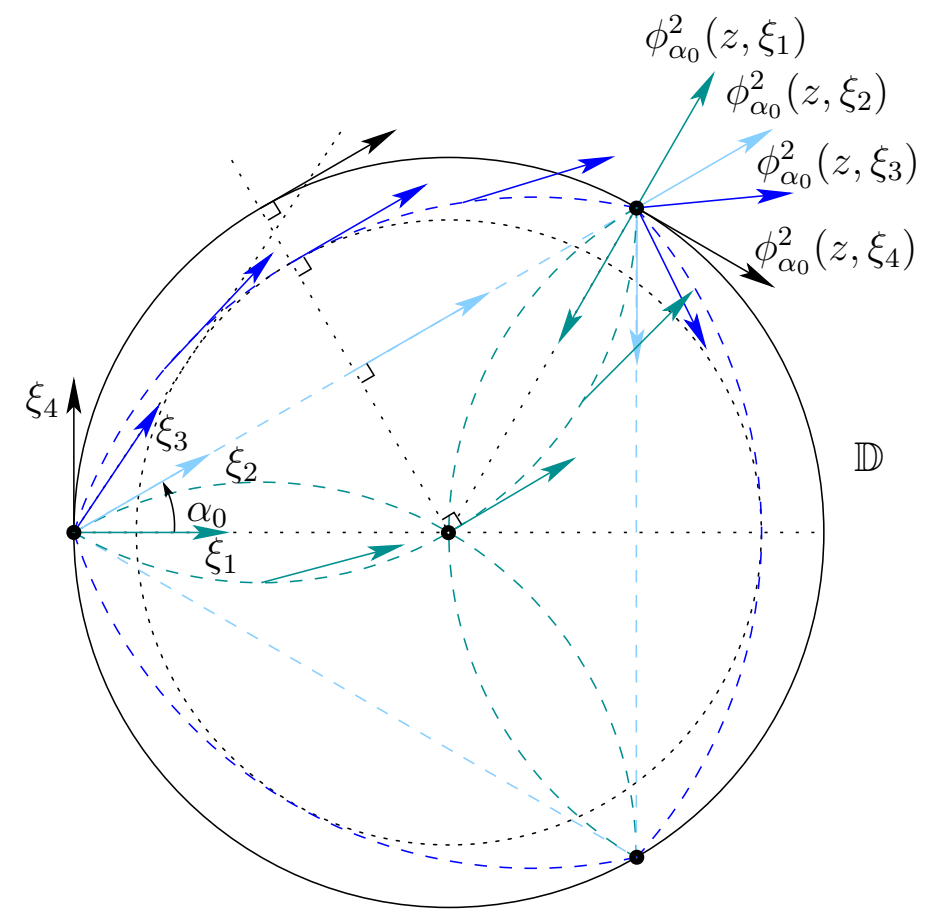

Figure 4. Approximate representation of some trajectories of the flow $\phi_{\alpha_{0}}^{\tau}$ with $\alpha_{0}=\pi / 6$ issued from $\left(z, \xi_{j}\right)$ with $z=(-1,0)$ and $\xi_{j}, j \in\{1,2,3,4\}$ such that $\alpha\left(z, \xi_{1}\right)=0, \alpha\left(z, \xi_{2}\right)=\alpha_{0}, \alpha\left(z, \xi_{3}\right) \in\left(\alpha_{0}, \pi / 2\right)$ and $\alpha\left(z, \xi_{4}\right)=\pi / 2$.

\section{Second microlocalization on a rational angle}

This section and the next one are devoted to proving the semiclassical version of our result, Theorem 2.4.

Let $\left(u_{h}^{0}\right)$ be a bounded family in $L^{2}(\mathbb{D})$. Denote by $u_{h}(z, t)=U_{V}(t) u_{h}^{0}(z)$ the corresponding solutions to (1.1). After extracting a subsequence, we suppose that its Wigner distributions $W_{h}$ (defined by (2.4)) converge to a semiclassical measure $\mu_{s c}$ in the weak-* topology of $\mathcal{D}^{\prime}\left(\mathbb{R}^{2} \times \mathbb{R}^{2} \times \mathbb{R}_{t} \times \mathbb{R}_{H}\right)$. The measure $\mu_{s c} \in L^{\infty}\left(\mathbb{R}_{t} ; \mathcal{M}_{+}\left(\mathbb{R}^{2} \times \mathbb{R}^{2} \times \mathbb{R}_{H}\right)\right)$ is for a.e. $t \in \mathbb{R}$ supported by $\overline{\mathbb{D}} \times \mathbb{R}^{2} \times \mathbb{R}_{H} \cap\left\{H=\frac{E^{2}}{2}\right\}$.

From now on, we skip the index $s c$ since there is no possible confusion here (only semiclassical measures are considered until Section 6) to lighten the notation.

The aim of this section is to understand the term $\mu\rceil_{\mathcal{I}_{\alpha_{0}}}$, where $\mathcal{I}_{\alpha_{0}}=\left\{\alpha=\alpha_{0}\right\}$ and $\alpha_{0} \in \pi \mathbb{Q}$. In view of Lemma 3.4, it suffices to characterize the action of $\mu\rceil_{\mathcal{I}_{\alpha_{0}}}$ on test functions that are $\left(\phi_{\alpha_{0}}^{\tau}\right)$-invariant.

4.1. Classes of test functions. Here is a list of properties that we may want to impose on our symbols in the course of our proof. We express these properties both in the "old" coordinates $\left(x, y, \xi_{x}, \xi_{y}\right)$ and in the "new" ones $(s, \theta, E, J)$.

Let $a$ be a smooth function of $\left(x, y, \xi_{x}, \xi_{y}, t, H\right)$, supported away from $\{\xi=0\}$. Then $a \circ \Phi$ is a smooth function of $(s, \theta, E, J, t, H)$ supported away from $\{E=0\}$. The properties we shall use are the following:

(A) The symbol $a$ is compactly supported w.r.t. $\xi, t$ and $H$. This is equivalent to $a \circ \Phi$ being compactly supported w.r.t. $E, J, t$ and $H$. Note also that $a \circ \Phi$ is $2 \pi$-periodic w.r.t. $\theta$.

(B) For $|z|=1$, we have $a(z, \xi)=a \circ \sigma(z, \xi)$ where $\sigma$ is the orthogonal symmetry with respect to the boundary of the disk at $z$. In the coordinates of Section 3.1, this reads (forgetting 
to write the $(t, H)$-dependence of $a)$

$$
a \circ \Phi(\cos \alpha, \theta, E, J)=a \circ \Phi(-\cos \alpha, \theta+\pi+2 \alpha, E, J)
$$

for all $\theta, E, J$ and for $\alpha=-\arcsin \left(\frac{J}{E}\right)$.

Terminology. We shall say that $a$ is a smooth function on $\mathbb{W}$ if $a$ is a smooth functon on $\overline{\mathbb{D}} \times \mathbb{R}^{2}$ that satisfies $(\mathrm{B})$.

(C) If $a$ satisfies (B), we want in addition that $a \circ \pi \circ \phi^{\tau}$ defines a smooth function on $\mathbb{W}$ for all $\tau$. This is equivalent to requiring that

$$
\partial_{s}^{k}(a \circ \Phi)(\cos \alpha, \theta, E, J)=\partial_{s}^{k}(a \circ \Phi)(-\cos \alpha, \theta+\pi+2 \alpha, E, J)
$$

for all $k$, for all $\theta, E, J$ and for $\alpha=-\arcsin \left(\frac{J}{E}\right)$. In other words, all the derivatives of $a \circ \Phi$ w.r.t. $s$ satisfy the symmetry condition (B).

(D) The function $a$ satisfies (C), and in addition $a$ is $\phi_{\alpha_{0}}^{\tau}$-invariant, which reads

$$
\left(\left(\alpha_{0}-\alpha\right) X_{P_{1}}+\frac{\cos \alpha}{E} X_{P_{0}}\right) a=0,
$$

or, in the new coordinates,

$$
\left[\left(\alpha_{0}-\alpha\right) \partial_{\theta}+\cos \alpha \partial_{s}\right] a \circ \Phi=0 .
$$

Furthermore, to fix ideas, let us assume that the support of $a$ with respect to $t$ is contained in $(-1,1)$. This implies that

$$
\begin{aligned}
W_{h}(a) & =\left\langle g(t) u_{h}, \mathrm{Op}_{1}\left(a\left(z, h \xi, t, h^{2} H\right) g(t) u_{h}\right\rangle_{L^{2}\left(\mathbb{R}^{2} \times \mathbb{R}\right)}+O\left(h^{\infty}\right)\right. \\
& =\left\langle g(t) u_{h}, \mathrm{Op}_{h}\left(a(z, \xi, t, h H) g(t) u_{h}\right\rangle_{L^{2}\left(\mathbb{R}^{2} \times \mathbb{R}\right)}+O\left(h^{\infty}\right),\right.
\end{aligned}
$$

for any smooth cut-off function $g$ supported in $(-2,2)$ and taking the value 1 on $(-1,1)$. In other words, we need only consider the restriction of $u_{h}(z, t)$ to $t \in(-2,2)$.

4.2. Coordinates adapted to the second microlocalization on $\mathcal{I}_{\alpha_{0}}$. We wish to study the concentration of $W_{h}$ around the set $\left\{J=-E \sin \alpha_{0}\right\}$. If the limit measure $\left(\Phi^{-1}\right)_{*} \mu$ is supported on the set $\{E=\sqrt{2 H}\}$ this is equivalent to studying the concentration of $W_{h}$ around $\{J=$ $\left.-\sqrt{2 H} \sin \alpha_{0}\right\}$. Since this assumption is satisfied for sequences $u_{h}$ satisfying (1.8), we shall study the concentration of $W_{h}$ around this set.

Thus we make the (symplectic) change of variables

$$
\left(s, \theta, E, J^{\prime}-\sqrt{2 H} \sin \alpha_{0}, t^{\prime}+\frac{\theta \sin \alpha_{0}}{\sqrt{2 H}}, H\right)=(s, \theta, E, J, t, H)
$$

which sends $\left\{J^{\prime}=0\right\}$ to $\left\{J=-\sqrt{2 H} \sin \alpha_{0}\right\}$ and leaves untouched the variables $(s, E)$.

Consider the following corresponding Fourier Integral Operator (which leaves untouched the variables $(s, E)$, omitted here from the notation):

$$
\mathscr{V} f(\theta, H)=(2 \pi)^{-1 / 2} e^{i \sqrt{2 H} \sin \alpha_{0} \theta / h} \int f(\theta, h t) e^{-i H t / h} d t .
$$

Lemma 4.1. If $b \in C_{c}^{\infty}\left(\mathbb{R}_{\theta} \times \mathbb{R}_{J} \times \mathbb{R}_{t} \times \mathbb{R}_{H}\right)$, we have

$$
\mathscr{V} \mathrm{Op}_{1}\left(b\left(\theta, h J, t, h^{2} H\right)\right) \mathscr{V}^{*}=\mathrm{Op}_{h}\left(\tilde{b}\left(\theta, J^{\prime}, H, h t\right)\right)+O(h),
$$

where $\tilde{b}\left(\theta, J^{\prime}, H, h t\right)=b\left(\theta, J^{\prime}-\sqrt{2 H} \sin \alpha_{0},-h t, H\right)$, and

$$
\mathscr{V}^{*}=I
$$

Proof. First notice that we have

$$
\mathscr{V}^{*} g(\theta, t)=(2 \pi)^{-1 / 2} h^{-1} \int g(\theta, H) e^{i H t / h^{2}} e^{-i \sqrt{2 H} \sin \alpha_{0} \theta / h} d H .
$$


Second, we may now compute $\left.A:=\mathscr{V} \mathrm{Op}_{1}\left(b\left(\theta, h J, t, h^{2} H\right)\right) \mathscr{V}^{*} e^{i t_{0} H / h} e^{i J_{0} \theta / h}\right\rceil_{H=H_{0}, \theta=\theta_{0}}$. We have the exact formula

$$
\begin{aligned}
A=(2 \pi h)^{-1} e^{i \sqrt{2 H_{0}} \sin \alpha_{0} \theta_{0} / h} \int b\left(\theta_{0}, J_{0}-\sqrt{2 H} \sin \alpha_{0}, h t, H\right) & \\
& e^{i H t / h} e^{i H t_{0} / h} e^{i J_{0} \theta_{0} / h} e^{-i \sqrt{2 H} \sin \alpha_{0} \theta_{0} / h} e^{-i H_{0} t / h} d H d t .
\end{aligned}
$$

Note that this expression is exact and thus does not involve the derivatives of $b$ w.r.t. $\theta$ or $J$. Taking $b=1$ in this expression gives the exact formula $A=e^{i t_{0} H_{0} / h} e^{i J_{0} \theta_{0} / h}$, which proves (4.3).

We carry on the computations with a general $b$. After a change of variables, $A$ is now equal to

$$
\begin{gathered}
A=(2 \pi h)^{-1} e^{i \sqrt{2 H_{0}} \sin \alpha_{0} \theta_{0} / h} e^{i J_{0} \theta_{0} / h} \int b\left(\theta_{0}, J_{0}-\sqrt{2 H} \sin \alpha_{0}, h t, H\right) \\
e^{i H\left(t+t_{0}\right) / h} e^{-i \sqrt{2 H} \sin \alpha_{0} \theta_{0} / h} e^{-i H_{0} t / h} d H d t \\
=(2 \pi h)^{-1} e^{i H_{0} t_{0} / h} e^{i \sqrt{2 H_{0}} \sin \alpha_{0} \theta_{0} / h} e^{i J_{0} \theta_{0} / h} \int b\left(\theta_{0}, J_{0}-\sqrt{2\left(H+H_{0}\right)} \sin \alpha_{0}, h\left(t-t_{0}\right), H_{0}+H\right) \\
e^{i H t / h} e^{-i \sqrt{2\left(H+H_{0}\right)} \sin \alpha_{0} \theta_{0} / h} d H d t .
\end{gathered}
$$

Standard application of the method of stationary phase shows that this expression is of order $O\left(h^{\infty}\right)$ if $H_{0}$ is away from the support of $b$. Besides, the phase has a single nondegenerate critical point at $(t, H)=\left(\frac{\sin \alpha_{0} \theta_{0}}{\sqrt{2 H_{0}}}, 0\right)$, so that uniformly in $t_{0} \in \mathbb{R}$ the method of stationary phase yields

$$
\begin{aligned}
A=(2 \pi h)^{-1} e^{i H_{0} t_{0} / h} e^{i \sqrt{2 H_{0}} \sin \alpha_{0} \theta_{0} / h} e^{i J_{0} \theta_{0} / h} & \\
& \left((2 \pi h) e^{-i \sqrt{2 H_{0}} \sin \alpha_{0} \theta_{0} / h} b\left(\theta_{0}, J_{0}-\sqrt{2 H_{0}} \sin \alpha_{0}, h\left(-t_{0}+\frac{\sin \alpha_{0} \theta_{0}}{\sqrt{2 H}}\right), H_{0}\right)\right)+O(h) .
\end{aligned}
$$

This is

$$
A=e^{i H_{0} t_{0} / h} e^{i J_{0} \theta_{0} / h} b\left(\theta_{0}, J_{0}-\sqrt{2 H_{0}} \sin \alpha_{0},-h t_{0}, H_{0}\right)+O(h),
$$

where $O(h)$ is uniform if $\theta_{0}$ stays in a fixed compact set. This concludes the proof of (4.2).

Recalling the definition of $W_{h}(a)$ in (2.4), we thus have

$$
\begin{aligned}
W_{h}(a) & =\left\langle\mathscr{V} \mathscr{U} u_{h},\left(\mathscr{V} \mathscr{U} \operatorname{Op}_{h}\left(a(z, \xi, t, h H) \mathscr{U}^{*} \mathscr{V}^{*}\right) \mathscr{V} \mathscr{U} u_{h}\right\rangle_{L^{2}\left(\mathbb{R}_{s} \times \mathbb{T}_{\theta} \times \mathbb{R}_{H}\right)}\right. \\
& =\left\langle\mathscr{V} \mathscr{U} u_{h}, \operatorname{Op}_{h}\left(\tilde{b}\left(s, \theta, J^{\prime}, E, H, h t\right)\right) \mathscr{V} \mathscr{U} u_{h}\right\rangle_{L^{2}\left(\mathbb{R}_{s} \times \mathbb{T}_{\theta} \times \mathbb{R}_{H}\right)}+O(h)
\end{aligned}
$$

where

$$
\left.\tilde{b}\left(s, \theta, J^{\prime}, E, H, h t\right)\right)=a \circ \Phi\left(s, \theta, E, J^{\prime}-\sin \alpha_{0} \sqrt{2 H},-h t, H\right),
$$

and $\mathbb{T}_{\theta}=\mathbb{R} / 2 \pi \mathbb{Z}$ is the circle in which the variable $\theta$ takes values. By (4.1), $u_{h}$ may actually be restricted to $|t|<2$ in this formula, so that it is safe to apply $\mathscr{V}$ to $\mathscr{U} u_{h}$.

To work in our new coordinates, we now define

$$
\left\langle w_{h}, b\right\rangle=\left\langle\mathscr{V} \mathscr{U} u_{h}, \mathrm{Op}_{h}\left(b\left(s, \theta, E, J^{\prime}, H, h t\right)\right) \mathscr{V} \mathscr{U} u_{h}\right\rangle_{L^{2}\left(\mathbb{R}_{s} \times \mathbb{T}_{\theta} \times \mathbb{R}_{H}\right)}
$$

for symbols $b$ that satisfies

(A) the symbol $b$ is compactly supported w.r.t. $E, J^{\prime}, t$ and $H$, and $2 \pi$-periodic w.r.t. $\theta$.

We then recover $W_{h}(a)=\left\langle w_{h}, \tilde{b}\right\rangle$ with $\tilde{b}$ and $a$ linked by (4.4).

Remark 4.2. Note that the bracket (4.5) can also be written as follows

$$
\left\langle w_{h}, b\right\rangle=\left\langle\mathscr{V} \mathscr{U} u_{h}, \mathrm{Op}_{h}\left(\chi_{0}(\theta) b\left(s, \theta, E, J^{\prime}, H, h t\right)\right) \mathscr{V} \mathscr{U} u_{h}\right\rangle_{L^{2}\left(\mathbb{R}_{s} \times \mathbb{R}_{\theta} \times \mathbb{R}_{H}\right)}
$$

for any $\chi_{0} \in C_{c}^{\infty}(\mathbb{R})$ satisfying $\sum_{k \in \mathbb{Z}} \chi_{0}(\theta+2 \pi k) \equiv 1$ on $\mathbb{R}$. Indeed, we have

$$
\mathrm{Op}_{h}\left(\chi_{0}(\theta) b\right)=\chi_{0}(\theta) \mathrm{Op}_{h}(b)
$$

(because $\mathrm{Op}$ denotes the standard quantization) and we write for any $2 \pi$-periodic function $f \in$ $L_{\text {loc }}^{1}(\mathbb{R}), \int_{\mathbb{R}} \chi_{0}(\theta) f(\theta) d \theta=\int_{\mathbb{T}} f(\theta) d \theta$. Because of $(4.7)$, we may also take $\chi_{0}=\mathbb{1}_{(0,2 \pi)}$ when needed. 
4.3. Second microlocalization. We now introduce two auxiliary distributions which describe more precisely how $w_{h}$ concentrates on the set

$$
\left.\left\{(s, \theta, E, J, H, t) \in \Phi^{-1}\left(\overline{\mathbb{D}} \times\left(\mathbb{R}^{2} \backslash\{0\}\right)\right)\right) \times \mathbb{R}^{2}, \text { such that }-\frac{J}{\sqrt{2 H}}=\sin \alpha_{0}\right\}
$$

(which intersection with $\{E=\sqrt{2 H}\}$ is equal to $\mathcal{I}_{\alpha_{0}} \cap\{E=\sqrt{2 H}\}$ ).

For this, we define an appropriate class of symbols depending on an additional variable $\eta$, which later in the calculations will be identified with $\frac{J^{\prime}}{h}=\frac{J+\sqrt{2 H} \sin \alpha_{0}}{h}$.

Definition 4.3. - We denote by $\mathcal{S}$ the class of smooth functions $b\left(s, \theta, E, J^{\prime}, \eta, H, t\right)$ on $\mathbb{R}^{7}$, supported away from $\{E=0\}$ and that satisfy condition $(\mathrm{A})$ in the variables $\left(s, \theta, E, J^{\prime}, H, t\right)$, and, in addition,

(E) $b$ is homogeneous of degree zero at infinity in $\eta \in \mathbb{R}$. That is, there exist $R_{0}>0$ and $b_{\text {hom }} \in C^{\infty}\left(\mathbb{R}^{4} \times\{-1,+1\} \times \mathbb{R}^{2}\right)$ with

$b\left(s, \theta, E, J^{\prime}, \eta, H, t\right)=b_{\text {hom }}\left(s, \theta, E, J^{\prime}, \frac{\eta}{|\eta|}, H, t\right), \quad$ for $|\eta|>R_{0}$ and $\left(s, \theta, E, J^{\prime}, H, t\right) \in \mathbb{R}^{6}$.

- We denote by $\mathcal{S}^{\sigma}$ those symbols $b \in \mathcal{S}$ that satisfy conditions (B) and (C) (for all $H, t$ ):

(B) $b\left(\cos \alpha, \theta, E, J^{\prime}\right)=b\left(-\cos \alpha, \theta+\pi+2 \alpha, E, J^{\prime}\right)$

for all $\theta, E, J^{\prime}$, and for $\alpha=-\arcsin \left(\frac{J^{\prime}-\sqrt{2 H} \sin \alpha_{0}}{E}\right)$.

(C) $\partial_{s}^{k} b\left(\cos \alpha, \theta, E, J^{\prime}\right)=\partial_{s}^{k} b\left(-\cos \alpha, \theta+\pi+2 \alpha, E, J^{\prime}\right)$

for all $k$, for all $\theta, E, J^{\prime}$, and for $\alpha=-\arcsin \left(\frac{J^{\prime}-\sqrt{2 H} \sin \alpha_{0}}{E}\right)$.

- We denote by $\mathcal{S}_{\alpha_{0}}^{\sigma}$ those symbols $b \in \mathcal{S}^{\sigma}$ satisfying the invariance condition (D):

(D) $\left[\left(\alpha_{0}-\alpha\right) \partial_{\theta}+\cos \alpha \partial_{s}\right] b\left(s, \theta, E, J^{\prime}\right)=0$

for all $s, \theta, E, J^{\prime}$, and for $\alpha=-\arcsin \left(\frac{J^{\prime}-\sqrt{2 H} \sin \alpha_{0}}{E}\right)$.

Let $\chi \in C_{c}^{\infty}(\mathbb{R})$ be a nonnegative cut-off function that is identically equal to one near the origin and let $R>0$. For $b \in \mathcal{S}$, we define

$$
\begin{aligned}
\left\langle w_{h, R}^{\alpha_{0}}, b\right\rangle:= & \\
& \left\langle\mathscr{V} \mathscr{U} u_{h}, \mathrm{Op}_{h}\left(\left(1-\chi\left(\frac{J^{\prime}}{R h}\right)\right) \chi_{0}(\theta) b\left(s, \theta, E, J^{\prime}, \frac{J^{\prime}}{h}, H, h t\right)\right) \mathscr{V} \mathscr{U} u_{h}\right\rangle_{L^{2}\left(\mathbb{R}_{s} \times \mathbb{R}_{\theta} \times \mathbb{R}_{H}\right)}
\end{aligned}
$$

and

(4.8)

$$
\left\langle w_{\alpha_{0}, h, R}, b\right\rangle:=\left\langle\mathscr{V} \mathscr{U} u_{h}, \mathrm{Op}_{h}\left(\chi\left(\frac{J^{\prime}}{R h}\right) \chi_{0}(\theta) b\left(s, \theta, E, J^{\prime}, \frac{J^{\prime}}{h}, H, h t\right)\right) \mathscr{V} \mathscr{U} u_{h}\right\rangle_{L^{2}\left(\mathbb{R}_{s} \times \mathbb{R}_{\theta} \times \mathbb{R}_{H}\right)},
$$

The Calderón-Vaillancourt theorem [CV71] ensures that both $w_{h, R}^{\alpha_{0}}$ and $w_{\alpha_{0}, h, R}$ are bounded in $\mathcal{S}^{\prime}$. After possibly extracting subsequences, we have the existence of a limit: for every $b \in \mathcal{S}$,

$$
\left\langle\mu^{\alpha_{0}}, b\right\rangle:=\lim _{R \rightarrow \infty} \lim _{h \rightarrow 0^{+}}\left\langle w_{h, R}^{\alpha_{0}}, b\right\rangle
$$

and

$$
\left\langle\mu_{\alpha_{0}}, b\right\rangle:=\lim _{R \rightarrow \infty} \lim _{h \rightarrow 0^{+}}\left\langle w_{\alpha_{0}, h, R}, b\right\rangle .
$$

Positivity properties are described in the next proposition.

Proposition 4.4. (i) The distribution $\mu^{\alpha_{0}}$ is a nonnegative Radon measure. In addition, $\mu^{\alpha_{0}}$ is nonnegative, 0-homogeneous and supported at infinity in the variable $\eta$ (i.e., it vanishes when paired with a compactly supported function). As a consequence, $\mu^{\alpha_{0}}$ may be identified with a nonnegative measure on $\mathbb{R}^{4} \times\{-1,+1\} \times \mathbb{R}_{t} \times \mathbb{R}_{H}$.

(ii) The projection of $\mu_{\alpha_{0}}$ on $\mathbb{R}_{s, \theta, E, J^{\prime}}^{4} \times \mathbb{R}_{H, t}^{2}$ (that is, $\int_{\mathbb{R}} \mu_{\alpha_{0}}(d \eta)$ ) is a nonnegative measure, carried on $\left\{J^{\prime}=0\right\}$. 
Moreover, both $\mu_{\alpha_{0}}$ and $\mu^{\alpha_{0}}$ are carried by the set $\{E=\sqrt{2 H}\}$, as can be seen from (C.12) and (C.13). Note also that the argument of Proposition D.1 proves that $\mu^{\alpha_{0}}$ enjoys $L^{\infty}$ regularity in the time variable.

Proposition 4.4 (i) is proved at the beginning of Section 4.4, whereas (ii) shall be a consequence of Section 4.5.

Remark 4.5. If $a=a(z, \xi, t, H)$ is a function on $\overline{\mathbb{D}} \times \mathbb{R}^{2} \times \mathbb{R}^{2}$, let us define

$$
\begin{aligned}
& \left.m^{\alpha_{0}}(a):=\mu^{\alpha_{0}}\left(b \mathbb{1}_{J^{\prime}=0}\right)\right), \\
& m_{\alpha_{0}}(a):=\mu_{\alpha_{0}}(b) .
\end{aligned}
$$

where $b\left(s, \theta, E, J^{\prime}, H, t\right)=a \circ \Phi\left(s, \theta, E, J^{\prime}-\sin \alpha_{0} \sqrt{2 H},-t, H\right)$ (a function that does not depend on the additional variable $\eta)$. Then we have

$$
\mu\rceil_{\mathcal{I}_{\alpha_{0}}}=m^{\alpha_{0}}+m_{\alpha_{0}} .
$$

Thus, understanding $\mu\rceil_{\mathcal{I}_{\alpha_{0}}}$ amounts to understanding both $m^{\alpha_{0}}$ and $m_{\alpha_{0}}$, which we shall do by understanding the structure of $\mu^{\alpha_{0}}$ and $\mu_{\alpha_{0}}$.

The following proposition states that both distributions $\mu_{\alpha_{0}}$ and $\mu^{\alpha_{0}}$ are invariant under the billiard flow, as $\mu$.

Proposition 4.6. The distributions $\mu_{\alpha_{0}}$ and $\mu^{\alpha_{0}}$ enjoy the following property:

$$
\left\langle\mu_{\alpha_{0}}, E \partial_{s} b\right\rangle=0, \quad\left\langle\mu^{\alpha_{0}}, E \partial_{s} b\right\rangle=0
$$

for every $b \in \mathcal{S}^{\sigma}$.

Proof. We use as a "black-box" the technical calculations developed in Appendix A. The main point of these calculations is to understand how an operator of the form

$$
\mathscr{U}^{*} \mathrm{Op}_{h}(P(s, \theta, E, J, t, h H)) \mathscr{U}
$$

preserves or modifies the Dirichlet boundary condition, according to the properties of $P$ (the technical difficulty is that our new coordinates $(s, \theta, E, J)$, well-adapted to the dynamics, are not adapted to express the Dirichlet boundary condition).

In the proof of Proposition 4.6 for $\mu_{\alpha_{0}}$, we consider the function $P$

$$
P(s, \theta, E, J, t, H)=b\left(s, \theta, E, J+\sin \alpha_{0} \sqrt{2 H}, \frac{J+\sin \alpha_{0} \sqrt{2 H}}{h}, H,-t\right) \chi\left(\frac{J+\sin \alpha_{0} \sqrt{2 H}}{R h}\right) .
$$

To prove the result for $\mu^{\alpha_{0}}$, the argument is the same with the function

$$
\begin{aligned}
& \tilde{P}(s, \theta, E, J, t, H)= \\
& \quad b\left(s, \theta, E, J+\sin \alpha_{0} \sqrt{2 H}, \frac{J+\sin \alpha_{0} \sqrt{2 H}}{h}, H,-t\right)(1-\chi)\left(\frac{J+\sin \alpha_{0} \sqrt{2 H}}{R h}\right) .
\end{aligned}
$$

(1) The operator $\mathscr{U}^{*} \mathrm{Op}_{h}(P(s, \theta, E, J, t, h H)) \mathscr{U}$ is expressed as a pseudodifferential operator $\mathcal{A}_{E}(P)$ on $\mathbb{R}^{2}$ (modulo a small remainder) in polar coordinates

$$
z=(-r \sin u, r \cos u)
$$

Note that the polar coordinates are the ones adapted to our boundary problem, since the boundary is given by the equation $r=1$.

Thus we have

$$
\lim \left\langle u_{h}, \mathscr{U}^{*} \mathrm{Op}_{h}(P(s, \theta, E, J, t, h H)) \mathscr{U} u_{h}\right\rangle=\lim \left\langle u_{h}, \mathcal{A}_{E}(P) u_{h}\right\rangle .
$$

(2) We then introduce a pseudodifferential operator $\mathcal{A}_{H}(P)$, having the property that the symbols of $\mathcal{A}_{E}(P)$ and $\mathcal{A}_{H}(P)$ coincide on $\left\{|\xi|^{2}=2 H\right\}$. More precisely, we are able to prove (Lemma B.1)

$$
\lim \left\langle u_{h}, \mathcal{A}_{E}(P) u_{h}\right\rangle=\lim \left\langle u_{h}, \mathcal{A}_{H}(P) u_{h}\right\rangle .
$$


(3) The explicit expression of $\mathcal{A}_{H}(P)$ reads

$$
A\left(r, u, \sqrt{2 h D_{t}}, h D_{u}, t\right)+B\left(r, u, \sqrt{2 h D_{t}}, h D_{u}, t\right) \circ h D_{r}
$$

modulo terms of order $O(h)$, where $z=(-r \sin u, r \cos u)$ is the decomposition in polar coordinates. The functions $A, B, C, D$ are expressed explicitly in terms of $P$ in Proposition A.3. If $P$ satisfies the symmetry condition (B), then $B \equiv 0$ for $r=1$.

(4) Finally, we show in Proposition B.3 that

$$
\lim _{h \rightarrow 0}\left\langle u_{h}, \mathcal{A}_{H}\left(E \partial_{s} P\right) u_{h}\right\rangle=\lim _{h \rightarrow 0}\left\langle u_{h},\left[-\frac{i h \Delta}{2}, \mathcal{A}_{H}(P)\right] u_{h}\right\rangle
$$

where $\Delta$ is the laplacian on $\mathbb{R}^{2}$. On the other hand, if $P$ depends on $t$, we have

$$
\left[\partial_{t}, \mathcal{A}_{H}(P)\right]=\mathcal{A}_{H}\left(\partial_{t} P\right) \text {. }
$$

The proof of Proposition 4.6 now goes as follows (with $b$ and $P$ related by (4.11)):

$$
\begin{aligned}
\left\langle\mu_{\alpha_{0}}, E \partial_{s} b\right\rangle= & \lim _{R \rightarrow \infty} \lim _{h \rightarrow 0^{+}}\left\langle w_{\alpha_{0}, h, R}, b\right\rangle \\
= & \lim _{R \rightarrow \infty} \lim _{h \rightarrow 0^{+}} \\
& \left\langle\mathscr{V} \mathscr{U} u_{h}, \mathrm{Op}_{h}\left(E \partial_{s} P\left(s, \theta, E, J^{\prime}-\sin \alpha_{0} \sqrt{2 H}, H,-h t\right)\right) \mathscr{V} \mathscr{U} u_{h}\right\rangle_{L^{2}\left(\mathbb{R}_{s} \times \mathbb{T}_{\theta} \times \mathbb{R}_{H}\right)} \\
= & \lim _{R \rightarrow \infty} \lim _{h \rightarrow 0^{+}}\left\langle\mathscr{U} u_{h}, \mathrm{Op}_{h}\left(E \partial_{s} P(s, \theta, E, J, t, h H)\right) \mathscr{U} u_{h}\right\rangle_{L^{2}\left(\mathbb{R}_{s} \times \mathbb{T}_{\theta} \times \mathbb{R}_{t}\right)} \\
= & \lim _{R \rightarrow \infty} \lim _{h \rightarrow 0^{+}}\left\langle u_{h}, \mathcal{A}_{H}\left(E \partial_{s} P\right) u_{h}\right\rangle \\
= & \lim _{R \rightarrow \infty} \lim _{h \rightarrow 0^{+}}\left\langle u_{h},\left[-\frac{i h}{2} \Delta, \mathcal{A}_{H}(P)\right] u_{h}\right\rangle \\
= & \lim _{R \rightarrow \infty} \lim _{h \rightarrow 0^{+}}\left\langle u_{h},\left[-\frac{i h}{2} \Delta+i h V-h \partial_{t}, \mathcal{A}_{H}(P)\right] u_{h}\right\rangle \\
= & \lim _{R \rightarrow \infty} \lim _{h \rightarrow 0^{+}}\left\langle\frac{i h}{2} \frac{\partial u_{h}}{\partial n} \otimes \delta_{\partial \mathbb{D}}, \mathcal{A}_{H}(P) u_{h}\right\rangle-\lim \left\langle u_{h}, \mathcal{A}_{H}(P) \frac{i h}{2} \frac{\partial u_{h}}{\partial n} \otimes \delta_{\partial \mathbb{D}}\right\rangle
\end{aligned}
$$

The last line comes from the fact that $u_{h}$, extended to $\mathbb{R}^{2}$ by the value 0 outside $\mathbb{D}$, satisfies

$$
\left(-\frac{i h}{2} \Delta+i h V-h \partial_{t}\right) u_{h}=\frac{i h}{2} \frac{\partial u_{h}}{\partial n} \otimes \delta_{\partial \mathbb{D}}
$$

where $\Delta$ is the laplacian on $\mathbb{R}^{2}$.

We use now the explicit expression (4.13) of $\mathcal{A}_{H}(P)$, modulo terms that vanish at the limit. Using the fact that $u_{h}$ satisfies Dirichlet boundary conditions, and the fact that $B$ vanishes for $r=1$ if $P$ satisfies the symmetry condition (B), we see that the last line (4.15) vanishes.

Note that only the limit $h \longrightarrow 0$ was actually used, so that the result holds even before taking the limit $R \longrightarrow+\infty$.

Remark 4.7. More generally, let $b(s, \theta, E, J, \eta, H, t)$ be a smooth function on $\mathbb{R} \times \mathbb{R} / 2 \pi \mathbb{Z} \times \mathbb{R}^{5}$ with bounded deratives, and compactly supported w.r.t. $s, E, J, H, t$. Let $\mathbf{P}(s, \theta, E, J, t, H)=$ $b\left(s, \theta, E, J+\sin \alpha_{0} \sqrt{2 H}, \frac{J+\sin \alpha_{0} \sqrt{2 H}}{h}, H,-t\right)$. Then, the same proof yields, without using the symmetry condition (B), the formula

$$
\begin{gathered}
\lim _{h}\left\langle\mathscr{V} \mathscr{U} u_{h}, \mathrm{Op}_{h}\left(E \partial_{s} b\left(s, \theta, E, J^{\prime}, \frac{J^{\prime}}{h}, H, h t\right)\right) \mathscr{V} \mathscr{U} u_{h}\right\rangle_{L^{2}\left(\mathbb{R}_{s} \times \mathbb{T}_{\theta} \times \mathbb{R}_{H}\right)} \\
=\lim _{h}\left\langle\mathscr{U} u_{h}, \mathrm{Op}_{h}\left(E \partial_{s} \mathbf{P}(s, \theta, E, J, t, h H)\right) \mathscr{U} u_{h}\right\rangle_{L^{2}\left(\mathbb{R}_{s} \times \mathbb{T}_{\theta} \times \mathbb{R}_{t}\right)} \\
=-\lim _{h}\left\langle h \frac{\partial u_{h}}{\partial n}, \mathbf{B}\left(1, u, \sqrt{2 h D_{t}}, h D_{u}, t\right) h \frac{\partial u_{h}}{\partial n}\right\rangle_{L^{2}(\partial \mathbb{D} \times \mathbb{R})}
\end{gathered}
$$

where $\mathbf{B}$ is the function associated to $\mathbf{P}$ by the formulas of Proposition A.3. Again, if $\mathbf{P}$ satisfies (B), the operator $\mathbf{B}\left(1, u, \sqrt{2 h D_{t}}, h D_{u}, t\right)$ vanishes. 
This formula, relating the semiclassical measures of boundary data to the semiclassical measures of interior data, is analogous to formula (2.22) but is expressed in a different set of coordinates.

Applying (4.17) to $\frac{s}{E} \mathbf{P}$ instead of $\mathbf{P}$ (that is, $\frac{s}{E} b$ instead of b) has the following consequence that will be used later

$$
\begin{aligned}
\lim _{h}\left\langle\mathscr{U} u_{h}, \mathrm{Op}_{h}\left(\left(\mathbf{P}+s \partial_{s} \mathbf{P}\right)(s, \theta, E, J, t, h H)\right) \mathscr{U} u_{h}\right\rangle_{L^{2}\left(\mathbb{R}_{s} \times \mathbb{T}_{\theta} \times \mathbb{R}_{t}\right)} & \\
= & -\lim _{h}\left\langle h \frac{\partial u_{h}}{\partial n},\left(E^{-2} \mathbf{P}\right)^{\sigma}\left(1, u, \sqrt{2 h D_{t}}, h D_{u}, t\right) h \frac{\partial u_{h}}{\partial n}\right\rangle_{L^{2}(\partial \mathbb{D} \times \mathbb{R})}
\end{aligned}
$$

with the notation (A.4).

The following result states that both $\mu^{\alpha_{0}}$ and $\mu_{\alpha_{0}}$ have some extra regularity (for two different reasons).

Theorem 4.8. $\quad$ (i) The measure $\mu^{\alpha_{0}}$ satisfies the invariance property:

$$
\left\langle\mu^{\alpha_{0}}, \partial_{\theta} b\right\rangle=0, \quad \text { for every } b \text { in } \mathcal{S}_{\alpha_{0}}^{\sigma} \text {. }
$$

(ii) The distribution $\mu_{\alpha_{0}}$ is concentrated on $\left\{J^{\prime}=0\right\}$ and its projection onto the variables $(s, \theta)$ is a nonnegative absolutely continuous measure.

Section 4.4 is devoted to the study of the properties of $\mu^{\alpha_{0}}$ and gives the proofs of Proposition 4.4 (i) and Theorem 4.8 (i). The study of the structure of $\mu_{\alpha_{0}}$ is performed in Section 4.5 using the notion of second-microlocal measures. This structure will imply (2.12) in Theorem 2.4 (iii). In particular, we prove at the end of Section 4.5 that it yields Theorem 4.8 (ii).

4.4. Structure and propagation of $\mu^{\alpha_{0}}$. In this section, we prove Proposition 4.4 (i) and the invariance property given by Theorem 4.8 (i).

The positivity of $\mu^{\alpha_{0}}$ can be deduced following the lines of [FKG02] Section 2.1, or those of the proof of Theorem 1 in [Gér91b]; or also Corollary 27 in [AM14]. The argument will not be reproduced here. Given $b \in \mathcal{S}$ there exists $R_{0}>0$ and $b_{\text {hom }} \in C_{c}^{\infty}\left(\mathbb{R}^{4} \times\{-1,+1\} \times \mathbb{R}^{2}\right)$ such that

$$
b\left(s, \theta, E, J^{\prime}, \eta, H, t\right)=b_{\mathrm{hom}}\left(s, \theta, E, J^{\prime}, \frac{\eta}{|\eta|}, H, t\right), \quad \text { for }|\eta| \geq R_{0}
$$

Clearly, for $R$ large enough, the value $\left\langle w_{h, R}^{\alpha_{0}}, b\right\rangle$ only depends on $b_{\text {hom. }}$ Therefore, the limiting distribution $\mu^{\alpha_{0}}$ can be viewed as an element of the dual space of $C_{c}^{\infty}\left(\mathbb{R}^{4} \times\{-1,+1\} \times \mathbb{R}^{2}\right)$. Its positivity implies that it is a measure, which proves Proposition 4.4 (i).

We now assume that $b \in \mathcal{S}_{\alpha_{0}}^{\sigma}$ and prove the invariance property Theorem 4.8 (i).

Let $b \in \mathcal{S}_{\alpha_{0}}^{\sigma}$, and define $\tilde{P}$ as in formula (4.12). Because of property (D) in the definition of the class $\mathcal{S}_{\alpha_{0}}^{\sigma}$, we have:

$$
\partial_{\theta} \tilde{P}(s, \theta, \sqrt{2 H}, J, t, H)=-\frac{\cos \alpha}{\alpha_{0}-\alpha} \partial_{s} \tilde{P}(s, \theta, \sqrt{2 H}, J, H, t)
$$

where $\alpha=-\arcsin \left(\frac{J}{\sqrt{2 H}}\right)$. The crucial point in what follows is that $\left|\frac{\cos \alpha}{\alpha_{0}-\alpha}\right| \leq \frac{C}{h R}$ on the support of $\tilde{P}(s, \theta, \sqrt{2 H}, J, t, H)$.

Recall that by definition

$$
\left\langle\mu^{\alpha_{0}}, \partial_{\theta} b\right\rangle=\lim _{R \rightarrow \infty} \lim _{h \rightarrow 0^{+}}\left\langle w_{h, R}^{\alpha_{0}}, \partial_{\theta} b\right\rangle .
$$


Let us first fix $R$ and study the limit $h \longrightarrow 0$. Arguing as in the proof of Proposition 4.6, we have

$$
\begin{aligned}
\lim _{h \rightarrow 0^{+}}\left\langle w_{h, R}^{\alpha_{0}}, \partial_{\theta} b\right\rangle= & \lim \left\langle\mathscr{U} u_{h}, \operatorname{Op}_{h}\left(\partial_{\theta} \tilde{P}(s, \theta, E, J, t, h H)\right) \mathscr{U} u_{h}\right\rangle_{L^{2}\left(\mathbb{R}_{s} \times \mathbb{T}_{\theta} \times \mathbb{R}_{H}\right)} \\
= & \lim \left\langle u_{h}, \mathcal{A}_{E}\left(\partial_{\theta} \tilde{P}\right) u_{h}\right\rangle \\
= & \lim \left\langle u_{h}, \mathcal{A}_{H}\left(\partial_{\theta} \tilde{P}\right) u_{h}\right\rangle \\
= & \lim \left\langle u_{h}, \mathcal{A}_{H}\left(-\frac{\cos \alpha}{\left(\alpha_{0}-\alpha\right)} \partial_{s} \tilde{P}\right) u_{h}\right\rangle \\
= & \lim \left\langle u_{h},\left[-\frac{i h}{2} \Delta+i h V-h \partial_{t}, \mathcal{A}_{H}\left(-\frac{\cos \alpha}{E\left(\alpha_{0}-\alpha\right)} \tilde{P}\right)\right] u_{h}\right\rangle+O\left(R^{-1}\right) \\
= & \lim \left\langle\frac{i h}{2} \frac{\partial u_{h}}{\partial n} \otimes \delta_{\partial \mathbb{D}}, \mathcal{A}_{H}\left(-\frac{\cos \alpha}{E\left(\alpha_{0}-\alpha\right)} \tilde{P}\right) u_{h}\right\rangle \\
& \quad-\lim \left\langle u_{h}, \mathcal{A}_{H}\left(-\frac{\cos \alpha}{E\left(\alpha_{0}-\alpha\right)} \tilde{P}\right) \frac{i h}{2} \frac{\partial u_{h}}{\partial n} \otimes \delta_{\partial \mathbb{D}}\right\rangle+O\left(R^{-1}\right)
\end{aligned}
$$

where we used again (4.16).

But $\mathcal{A}_{H}\left(-\frac{\cos \alpha}{E\left(\alpha_{0}-\alpha\right)} \tilde{P}\right)$ equals (modulo terms which only add an error $O\left(R^{-1}\right)$ to the whole calculation)

$$
\begin{aligned}
& -\tilde{A}\left(r, u, \sqrt{2 h D_{t}}, h D_{u}, t\right) \frac{\cos \alpha\left(h D_{u}, h D_{t}\right)}{\sqrt{2 h D_{t}}\left(\alpha_{0}-\alpha\left(h D_{u}, h D_{t}\right)\right)} \\
& \quad-\tilde{B}\left(r, u, \sqrt{2 h D_{t}}, h D_{u}, t\right) \frac{\cos \alpha\left(h D_{u}, h D_{t}\right)}{\sqrt{2 h D_{t}}\left(\alpha_{0}-\alpha\left(h D_{u}, h D_{t}\right)\right)} \circ h D_{r}
\end{aligned}
$$

where $z=(-r \sin u, r \cos u)$ is the decomposition in polar coordinates, and $\tilde{A}, \tilde{B}$ are the functions associated to $\tilde{P}$ by the formulas of Proposition A.3.

If $\tilde{P}$ satisfies (B) then $\tilde{B} \equiv 0$ for $r=1$. Since $u_{h}$ satisfies Dirichlet boundary conditions, we see that the last terms in (4.20) vanish.

To conclude the proof, we take $R \longrightarrow+\infty$ after taking $h \longrightarrow 0$, so that the terms estimated as $O\left(R^{-1}\right)$ vanish.

4.5. Second microlocal structure of $\mu_{\alpha_{0}}$. If $\mathcal{H}$ is a Hilbert space, we shall denote by $\mathcal{L}(\mathcal{H})$, $\mathcal{K}(\mathcal{H})$ and $\mathcal{L}^{1}(\mathcal{H})$ the spaces of bounded, compact and trace class operators on $\mathcal{H}$. It is well known that $\mathcal{L}^{1}(\mathcal{H})$ is the dual of $\mathcal{K}(\mathcal{H})$. A measure on a polish space $T$, taking values in $\mathcal{L}^{1}(H)$, is defined as a bounded linear functional $\rho$ from $C_{c}(T)$ to $\mathcal{L}^{1}(H) ; \rho$ is said to be nonnegative if, for every nonnegative $b \in C_{c}(T), \rho(b)$ is a nonnegative hermitian operator. The set of such measures is denoted by $\mathcal{M}_{+}\left(T ; \mathcal{L}^{1}(H)\right)$; they can be identified in a natural way to nonnegative linear functionals on $C_{c}(T ; \mathcal{K}(H))$. Background and further details on operator-valued measures may be found for instance in [Gér91b].

For each $\omega \in \mathbb{R} / 2 \pi \mathbb{Z}$, let us define $\mathcal{H}_{\omega}$, the space of functions $f$ on $\mathbb{R}$ satisfying $f(\theta+2 \pi)=$ $f(\theta) e^{i \omega}$ and that are square-integrable on $(0,2 \pi)$.

We shall denote by $\mathcal{K}^{2 \pi}$ the space of operators on $L^{2}(\mathbb{R})$ whose kernel $K$ satisfies $K\left(\theta+2 \pi, \theta^{\prime}+\right.$ $2 \pi)=K\left(\theta, \theta^{\prime}\right)$ and that define compact operators on each $\mathcal{H}_{\omega}$. Each Hilbert space $\mathcal{H}_{\omega}$ is isometric to $L^{2}(0,2 \pi)$ (just by restricting functions to $(0,2 \pi)$ ), and in this identification the kernel of $K$ acting on $\mathcal{H}_{\omega}$ is given by

$$
K_{\omega}\left(\theta, \theta^{\prime}\right):=\mathbb{1}_{(0,2 \pi)}(\theta) \mathbb{1}_{(0,2 \pi)}\left(\theta^{\prime}\right) \sum_{n \in \mathbb{Z}} K\left(\theta, \theta^{\prime}+2 \pi n\right) e^{i n \omega} .
$$

The idea of the Floquet-Bloch theory is that it is completely equivalent to know $K\left(\theta, \theta^{\prime}\right)$ and to know $K_{\omega}\left(\theta, \theta^{\prime}\right)$ for almost all $\omega$, by decomposing

$$
L^{2}(\mathbb{R})=\int_{\oplus} \mathcal{H}_{\omega} d \omega
$$


Besides, $K$ is a nonnegative (resp. bounded) operator if and only if $K_{\omega}$ is nonnegative (resp. bounded) for a.e. $\omega$.

An example of an operator in $\mathcal{K}^{2 \pi}$ is

$$
K_{b, h, R}(s, E, H, t)=b\left(s, \theta, E, h D_{\theta}, D_{\theta}, H, t\right) \chi\left(D_{\theta} / R\right)
$$

for $b \in \mathcal{S}$ and fixed $R, h, t, H, s, E$. Note that, as $h \longrightarrow 0$, we have $K_{b, h, R}(s, E, H, t)=$ $K_{b, 0, R}(s, E, H, t)+O_{R}(h)$.

If $b$ satisfies the symmetry condition (B), note that the operator $K_{b, 0, R}(s, E, H, t)$ has the property

$$
K(\cos \alpha, E, H, t)=R_{\pi+2 \alpha}^{-1} \circ K(-\cos \alpha, E, H, t) \circ R_{\pi+2 \alpha}
$$

where $R$ is a translation operator on $L^{2}\left(\mathbb{R}_{\theta}\right): R_{\alpha} f(\theta)=f(\theta-\alpha)$ and where $\alpha=\arcsin \left(\frac{\sin \alpha_{0} \sqrt{2 H}}{E}\right)$. In particular,

$$
K\left(\cos \alpha_{0}, \sqrt{2 H}, H, t\right)=R_{\pi+2 \alpha_{0}}^{-1} \circ K\left(-\cos \alpha_{0}, \sqrt{2 H}, H, t\right) \circ R_{\pi+2 \alpha_{0}}
$$

Remark 4.9. The fact that the orbits of the billiard flow are periodic on $\mathcal{I}_{\alpha_{0}}\left(\alpha_{0} \in \pi \mathbb{Q}\right)$ is reflected in the fact that the function $s \mapsto K(s, \sqrt{2 H}, H, t)$ is periodic, if $K$ satisfies (4.24).

For $K \in C_{c}^{\infty}\left(\mathbb{R} / 2 \pi \mathbb{Z} \times \mathbb{R}_{s} \times \mathbb{R}_{E} \times \mathbb{R}_{H} \times \mathbb{R}_{t} ; \mathcal{K}\left(L^{2}(0,2 \pi)\right)\right)$, let us define:

$$
\begin{aligned}
&\left\langle n_{h}^{\alpha_{0}}, K\right\rangle=(2 \pi h)^{-2} \int_{\omega=0}^{2 \pi} \sum_{H, \sqrt{2 H} \sin \alpha_{0} / h \equiv \omega(2 \pi)} \frac{h}{\sin \alpha_{0}} \sqrt{\frac{H}{2}} \int_{s, s^{\prime}, E, H^{\prime}, t} \\
&\left\langle\chi_{0} \mathscr{V} \mathscr{U} u_{h}\left(s^{\prime}, H^{\prime}\right), K\left(\omega, s, E, H^{\prime}, h t\right) \chi_{0} \mathscr{V} \mathscr{U} u_{h}(s, H)\right\rangle_{L^{2}(0,2 \pi)} \\
& e^{i E\left(s^{\prime}-s\right) / h} e^{i t\left(H^{\prime}-H\right) / h} d s d E d s^{\prime} d H^{\prime} d t
\end{aligned}
$$

where $\chi_{0}$ is $\mathbb{1}_{(0,2 \pi)}$ as in Remark 4.2. This is also

$$
\left\langle\chi_{0} \mathscr{V} \mathscr{U} u_{h}, \mathcal{K} \chi_{0} \mathscr{V} \mathscr{U} u_{h}\right\rangle_{L^{2}\left(\mathbb{R}_{s} \times \mathbb{R}_{H}, L^{2}(0,2 \pi)\right)}
$$

where $\mathcal{K}$ is the pseudodifferential operator with operator-valued symbol:

$$
\int_{\omega=0}^{2 \pi} \sum_{H, \sqrt{2 H} \sin \alpha_{0} / h \equiv \omega(2 \pi)} \frac{h}{\sin \alpha_{0}} \sqrt{\frac{H}{2}} K\left(\omega, s, E, H^{\prime}, h t\right) .
$$

Remark 4.10. As noted earlier, it is equivalent (by the relation (4.21)) to consider a family $K(\omega)$ of kernels on $(0,2 \pi)^{2}$ and a kernel $K$ on $\mathbb{R}^{2}$ satisfying $K\left(\theta, \theta^{\prime}\right)=K\left(\theta+2 \pi, \theta^{\prime}+2 \pi\right)$. With this identification in mind, formula (4.25) amounts to

$$
\begin{gathered}
(2 \pi h)^{-2} \int_{s, s^{\prime}, E, H, H^{\prime}, t}\left\langle\chi_{0} \mathscr{V} \mathscr{U} u_{h}\left(s^{\prime}, H^{\prime}\right), K\left(s, E, H^{\prime}, h t\right) \mathscr{V} \mathscr{U} u_{h}(s, H)\right\rangle_{L^{2}(\mathbb{R})} \\
e^{i E\left(s^{\prime}-s\right) / h} e^{i t\left(H^{\prime}-H\right) / h} d s d E d s^{\prime} d H d H^{\prime} d t \\
=\left\langle\chi_{0} \mathscr{V} \mathscr{U} u_{h}, K\left(s, h D_{s}, H, h^{2} D_{t}\right) \mathscr{V} \mathscr{U} u_{h}\right\rangle_{L^{2}\left(\mathbb{R}_{s} \times \mathbb{R}_{H}, L^{2}\left(\mathbb{R}_{\theta}\right)\right)}
\end{gathered}
$$

The motivation for rewriting (4.27) in the apparently more complicated form (4.25) is that it will be more convenient to use the compact operators $K(\omega)$ on each $\mathcal{H}_{\omega}$ than the non-compact operator $K$ on $L^{2}(\mathbb{R})$.

The relevance of definition (4.25) for us is that we have the relation

$$
\begin{aligned}
\left\langle w_{\alpha_{0}, h, R}, b\right\rangle & =\left\langle n_{h}^{\alpha_{0}}, K_{b, h, R}\right\rangle \\
& =\left\langle n_{h}^{\alpha_{0}}, K_{b, 0, R}\right\rangle+O_{R}(h)
\end{aligned}
$$

where $K_{b, h, R}$ was defined in (4.22). 
Proposition 4.11. Suppose $\left(u_{h}^{0}\right)$ is bounded in $L^{2}(\mathbb{D})$. Then, modulo taking subsequences, the following convergence takes place:

$$
\lim _{h \rightarrow 0^{+}}\left\langle n_{h}^{\alpha_{0}}, K\right\rangle=\int_{0}^{2 \pi} \int_{\mathbb{R}_{s} \times \mathbb{R}_{E} \times \mathbb{R}_{H} \times \mathbb{R}_{t}} \operatorname{Tr}\left\{K(\omega, s, E, H, t) \rho_{\alpha_{0}}(d \omega, d s, d E, d H, d t)\right\},
$$

for every $K \in C_{c}^{\infty}\left(\mathbb{R} / 2 \pi \mathbb{Z} \times \mathbb{R}_{s} \times \mathbb{R}_{E} \times \mathbb{R}_{H} \times \mathbb{R}_{t} ; \mathcal{K}\left(L^{2}(0,2 \pi)\right)\right)$. In other words, $\rho_{\alpha_{0}}$ is the limit of $n_{h}^{\alpha_{0}}$ in the weak-* topology of

$$
\mathcal{D}^{\prime}\left(\mathbb{R} / 2 \pi \mathbb{Z} \times \mathbb{R}_{s} \times \mathbb{R}_{E} \times \mathbb{R}_{H} \times \mathbb{R}_{t}, \mathcal{L}^{1}\left(L^{2}(0,2 \pi)\right)\right) .
$$

In fact, $\rho_{\alpha_{0}}$ is a nonnegative, $\mathcal{L}^{1}\left(L^{2}(0,2 \pi)\right)$-valued measures on $\mathbb{R} / 2 \pi \mathbb{Z} \times \mathbb{R}_{s} \times \mathbb{R}_{E} \times \mathbb{R}_{H} \times \mathbb{R}_{t}$. In addition, $\rho_{\alpha_{0}}$ is supported in $\left\{s \in\left[-\cos \alpha_{0}, \cos \alpha_{0}\right], E=\sqrt{2 H}\right\}$.

Proof. Note that $\chi_{0} \mathscr{V} \mathscr{U} u_{h}(s, H)$ is bounded in $L^{2}\left(\mathbb{R}_{s} \times \mathbb{R}_{H}, L^{2}(0,2 \pi)\right)$. The Calderón-Vaillancourt theorem [CV71] gives that the operators $\mathcal{K}$ with symbols of the form (4.26) are uniformly bounded with respect to $h$. Therefore, the linear map

$$
L_{h}: K \mapsto \int_{\mathbb{R}}\left\langle n_{h}^{\alpha_{0}}, K\right\rangle
$$

is uniformly bounded as $h \longrightarrow 0$. As a consequence, for any $K$, up to extraction of a subsequence, it has a limit $l(K)$.

Considering a countable dense subset of $C_{c}^{\infty}\left(\mathbb{R} / 2 \pi \mathbb{Z} \times \mathbb{R}_{s} \times \mathbb{R}_{E} \times \mathbb{R}_{H} \times \mathbb{R}_{t} ; \mathcal{K}\left(L^{2}(0,2 \pi)\right)\right)$, and using a diagonal extraction process, one finds a sequence $\left(h_{n}\right)$ tending to 0 as $n$ goes to $+\infty$ such that for any $K \in C_{c}^{\infty}\left(\mathbb{R} / 2 \pi \mathbb{Z} \times \mathbb{R}_{s} \times \mathbb{R}_{E} \times \mathbb{R}_{H} \times \mathbb{R}_{t} ; \mathcal{K}\left(L^{2}(0,2 \pi)\right)\right)$, the sequence $L_{h_{n}}(K)$ has a limit as $n$ goes to $+\infty$.

The limit is a linear form on $C_{c}^{\infty}\left(\mathbb{R} / 2 \pi \mathbb{Z} \times \mathbb{R}_{s} \times \mathbb{R}_{E} \times \mathbb{R}_{H} \times \mathbb{R}_{t} ; \mathcal{K}\left(L^{2}(0,2 \pi)\right)\right)$, characterized by an element $\rho_{\alpha_{0}}$ of the dual space $\mathcal{D}^{\prime}\left(\mathbb{R} / 2 \pi \mathbb{Z} \times \mathbb{R}_{s} \times \mathbb{R}_{E} \times \mathbb{R}_{H} \times \mathbb{R}_{t}, \mathcal{L}^{1}\left(L^{2}(0,2 \pi)\right)\right)$.

The positivity of the limit is standard. Note that it is immediately seen in the expression (4.27).

Comparing with (4.28), we obtain

Corollary 4.12. For every $b \in \mathcal{S}$,

$$
\begin{aligned}
\int b(s, \theta, E, J, \eta, H, t) \mu_{\alpha_{0}}(d s, d \theta, d J, & d \eta, d E, d H, d t) \\
& =\operatorname{Tr}_{L^{2}(0,2 \pi)} \int K_{b, 0, \infty}(s, E, H, t)_{\omega} \rho_{\alpha_{0}}(d \omega, d s, d E, d H, d t)
\end{aligned}
$$

Remember that $K_{b, 0, R}(s, E, H, t)=b\left(s, \theta, 0, D_{\theta}, H, t\right) \chi\left(D_{\theta} / R\right)$, so that $K_{b, 0, \infty}(s, E, H, t)=$ $b\left(s, \theta, 0, D_{\theta}, H, t\right)$.

Corollary 4.13. If $b$ does not depend on $\eta$ then the above identity can be rewritten as:

$$
\begin{aligned}
\int b(s, \theta, E, J, H, t) \mu_{\alpha_{0}}(d s, d \theta, d J, d \eta, d E, & d H, d t) \\
& =\operatorname{Tr}_{L^{2}(0,2 \pi)} \int m_{b}(s, E, H, t) \rho_{\alpha_{0}}(d \omega, d s, d E, d H, d t)
\end{aligned}
$$

where $m_{b}(s, E, H, t)$ is the multiplication operator by $b(s, \theta, E, 0, H, t)$ acting on $L^{2}(0,2 \pi)$.

Note that $\int b(s, \theta, E, J, H, t) \mu_{\alpha_{0}}(d s, d \theta, d J, d \eta, d E, d H, d t) \geq 0$ if $b$ does not depend on $\eta$ and $b \geq 0$. Thus the projection on $\mu_{\alpha_{0}}$ on the variables $(s, \theta, E, J, H, t)$ defines a nonnegative measure.

We finish this section by explaining why this implies that the projection of $\mu_{\alpha_{0}}$ on the variables $(s, \theta)$ is absolutely continuous. If $b \in \mathcal{S}^{\sigma}$ does not depend on $\eta$, Proposition 4.6 implies that

$$
\begin{aligned}
\int b(s, \theta, E, J, H, t) \mu_{\alpha_{0}}(d s, d \theta, d J, d \eta, d E & , d H, d t) \\
& =\int\langle b\rangle_{\alpha_{0}}(\theta, E, J, H, t) \mu_{\alpha_{0}}(d s, d \theta, d J, d \eta, d E, d H, d t) .
\end{aligned}
$$


We know from Section 2.8.2 that $\mu=\mu_{s c}$ does not charge the set $S$. Since $\mu_{\alpha_{0}} \leq \mu$ by (4.10), the measure $\mu_{\alpha_{0}}$ does not charge the set $\left\{s= \pm \cos \alpha_{0}\right\}$, and the previous equality actually holds for all $b \in \mathcal{S}$. If $b$ does not depend on $(E, J, H, t)$, we get the formula

$$
\int b(s, \theta) \mu_{\alpha_{0}}(d s, d \theta, d J, d \eta, d E, d H, d t)=\operatorname{Tr}_{L^{2}(0,2 \pi)} \int m_{\langle b\rangle_{\alpha_{0}}} \rho_{\alpha_{0}}(d \omega, d s, d E, d H, d t) .
$$

This formula, defined a priori for continuous $b$, extends to $b \in L^{\infty}$. If $b$ vanishes for Lebesguealmost every $(s, \theta)$, the multiplication operator $m_{\langle b\rangle_{\alpha_{0}}}$ vanishes on $L^{2}(0,2 \pi)$, and

$$
\int b(s, \theta) \mu_{\alpha_{0}}(d s, d \theta, d J, d \eta, d E, d H, d t)=0,
$$

which proves the absolute continuity.

4.6. Propagation law for $\rho_{\alpha_{0}}$. We now show that the operator-valued measure $\rho_{\alpha_{0}}$ constructed in the previous section possesses some invariance properties. Below, the notation $\langle V\rangle_{\alpha_{0}}$ stands short for the function $\langle V\rangle_{\alpha_{0}} \circ \Phi\left(s, \theta, E,-E \sin \alpha_{0}, t\right)$, a function that actually does not depend on $s$ and is $2 \pi$-periodic in $\theta$.

Proposition 4.14. (i) If $K$ satisfies (4.24), we have

$$
\int \operatorname{Tr} E \partial_{s} K(\omega, s, E, H, t) \rho_{\alpha_{0}}(d \omega, d s, d E, d H, d t)=0
$$

(ii) If in addition $K(s, \sqrt{2 H}, H, t)$ does not depend on $s$, we have

$$
\begin{aligned}
\int \operatorname{Tr}\left(-\cos ^{2} \alpha_{0} \partial_{t} K+i\left[-\frac{\partial_{\theta}^{2}}{2}+\cos ^{2} \alpha_{0}\langle V\rangle_{\alpha_{0}}, K\right]_{\omega}\right) & (\omega, s, E, H, t) \\
& \rho_{\alpha_{0}}(d \omega, d s, d E, d H, d t)=0
\end{aligned}
$$

where $\left[-\frac{\partial_{\theta}^{2}}{2}+\cos ^{2} \alpha_{0}\langle V\rangle_{\alpha_{0}}, K\right]_{\omega}$ means that we are considering $\partial_{\theta}^{2}$ acting on $\mathcal{H}_{\omega}$ (in other words, $L^{2}(0,2 \pi)$ with Floquet-periodic boundary condition $\left(f(\theta+2 \pi)=f(\theta) e^{i \omega}\right)$.

The proof of this key proposition is postponed to the end of this Section. Let us first draw some of its consequences in view of Theorem 2.4.

Remark 4.15. Proposition 4.14 (ii) implies the following. Take $K=a(\omega, E, H, t) I d_{L^{2}(0,2 \pi)}$ with a a scalar continous function, then

$$
\operatorname{Tr}\left(\int \partial_{t} a(\omega, E, H, t) \rho_{\alpha_{0}}(d \omega, d s, d E, d H, d t)\right)=0 .
$$

Therefore, the image of $\rho_{\alpha_{0}}$ by the projection on $\mathbb{R}_{s}$,

$$
\bar{\rho}_{\alpha_{0}}(d \omega, d E, d H, d t):=\int \rho_{\alpha_{0}}(d \omega, d s, d E, d H, d t)
$$

is such that $\operatorname{Tr}\left(\bar{\rho}_{\alpha_{0}}\right)$ does not depend on $t$.

Remark 4.16. The Radon-Nikodym theorem [Gér91b, Appendix] implies that the operator valued measure $\bar{\rho}_{\alpha_{0}}$ can also be written as $\bar{\rho}_{\alpha_{0}}=\sigma_{\alpha_{0}} \ell_{\alpha_{0}}$ where $\ell_{\alpha_{0}}=\operatorname{Tr}\left(\bar{\rho}_{\alpha_{0}}\right)$ is a nonnegative scalar measure on $\mathbb{R} / 2 \pi \mathbb{Z} \times \mathbb{R}_{E} \times \mathbb{R}_{H}$, and

$$
\sigma_{\alpha_{0}}:(\mathbb{R} / 2 \pi \mathbb{Z})_{\omega} \times \mathbb{R}_{E} \times \mathbb{R}_{H} \times \mathbb{R}_{t} \rightarrow \mathcal{L}_{+}^{1}\left(L^{2}(0,2 \pi)\right),
$$

is an integrable function with respect to $\ell_{\alpha_{0}}$, taking values in the set of nonnegative trace-class operators on $L^{2}(0,2 \pi)$. Note that $\operatorname{Tr}\left(\sigma_{\alpha_{0}}\right)=1$.

Corollary 4.17. Let $\bar{\rho}_{\alpha_{0}}$ as in Remark 4.15 and let $\ell_{\alpha_{0}}$ and $\sigma_{\alpha_{0}}$ as in Remark 4.16. Then for $\ell_{\alpha_{0}}$-almost every $(\omega, E, H)$, we have

$$
-\cos ^{2} \alpha_{0} \partial_{t} \sigma_{\alpha_{0}}+i\left[-\frac{\partial_{\theta}^{2}}{2}+\cos ^{2} \alpha_{0}\langle V\rangle_{\alpha_{0}}, \sigma_{\alpha_{0}}\right]_{\omega}=0
$$

in $\mathcal{D}^{\prime}\left(\mathbb{R}_{t} ; \mathcal{L}_{+}^{1}\left(L^{2}(0,2 \pi)\right)\right)$. 
Therefore, for $\ell_{\alpha_{0}}$-almost every $(\omega, E, H), \sigma_{\alpha_{0}}$ coincides with a continuous function in

$$
C^{0}\left(\mathbb{R}_{t} ; \mathcal{L}_{+}^{1}\left(L^{2}(0,2 \pi)\right)\right)
$$

and

$$
\sigma_{\alpha_{0}}(\omega, E, H, t)=U_{\alpha_{0}, \omega}(t) \sigma_{\alpha_{0}}(\omega, E, H, 0) U_{\alpha_{0}, \omega}^{*}(t)
$$

where $U_{\alpha_{0}, \omega}(t)$ is the unitary propagator of the equation

$$
-\cos ^{2} \alpha_{0} D_{t} v(t, \theta)+\left(-\frac{1}{2} \partial_{\theta}^{2}+\cos ^{2} \alpha_{0}\langle V\rangle_{\alpha_{0}} \circ \Phi\right) v(t, \theta)=0
$$

Proof. We first rewrite (4.31) for $s$-independent operators $K$ as

$$
\int \operatorname{Tr}\left\{\left(-\cos ^{2} \alpha_{0} \partial_{t} K+i\left[-\frac{\partial_{\theta}^{2}}{2}+\cos ^{2} \alpha_{0}\langle V\rangle_{\alpha_{0}}, K\right]_{\omega}\right) \sigma_{\alpha_{0}}\right\} \ell_{\alpha_{0}}(d \omega, d E, d H) d t=0 .
$$

Therefore, we have

$$
\int \operatorname{Tr}\left\{K\left(-\cos ^{2} \alpha_{0} \partial_{t} \sigma_{\alpha_{0}}+i\left[-\frac{\partial_{\theta}^{2}}{2}+\cos ^{2} \alpha_{0}\langle V\rangle_{\alpha_{0}}, \sigma_{\alpha_{0}}\right]_{\omega}\right)\right\} \ell_{\alpha_{0}}(d \omega, d E, d H) d t=0
$$

which concludes the proof of Corollary 4.17.

To conclude this section, let us now prove its main result.

Proof of Propostion 4.14. As was already mentioned, it is equivalent to consider a family of kernels depending on $\omega, K(\omega, s, E, H, t)\left(\theta, \theta^{\prime}\right)$ defined for $\left(\theta, \theta^{\prime}\right) \in(0,2 \pi)^{2}$, and a kernel $K(s, E, H, t)\left(\theta, \theta^{\prime}\right)$ defined for $\left(\theta, \theta^{\prime}\right) \in \mathbb{R}^{2}$ and satisfying $K(s, E, H, t)\left(\theta, \theta^{\prime}\right)=K(s, E, H, t)\left(\theta+2 \pi, \theta^{\prime}+2 \pi\right)$. The link between both representations is the formula

$$
K(\omega, s, E, H, t)\left(\theta, \theta^{\prime}\right)=\sum_{n \in \mathbb{Z}} K(s, E, H, t)\left(\theta, \theta^{\prime}+2 n \pi\right) e^{i n \omega} .
$$

By a density argument, it is enough to treat the case where $K(s, E, H, t)$ is smooth in $(s, E, H, t)$ and is a pseudodifferential operator on $L^{2}(\mathbb{R})$. By this, we mean that there is a $b_{0}(s, \theta, E, \eta, H, t) \in$ $C_{c}^{\infty}\left(\mathbb{R} \times \mathbb{R} / 2 \pi \mathbb{Z} \times \mathbb{R}^{4}\right)$ such that $K(s, E, H, t)=b_{0}\left(s, \theta, E, D_{\theta}, H, t\right)$. As $\rho_{\alpha_{0}}$ is supported by $\{E=\sqrt{2 H}\}$, we may further assume that $K$ satisfies (4.23) instead of (4.24).

If $K$ satisfies (4.23), then we have $b_{0}(\cos \alpha, \theta, E, \eta, H, t)=b_{0}(-\cos \alpha, \theta+\pi+2 \alpha, E, \eta, H, t)$ for $\alpha=\arcsin \left(\frac{\sqrt{2 H} \sin \alpha_{0}}{E}\right)$. We can extend $b_{0}$ to a function $b\left(s, \theta, E, J^{\prime}, \eta, H, t\right) \in C_{c}^{\infty}\left(\mathbb{R} \times \mathbb{R} / 2 \pi \mathbb{Z} \times \mathbb{R}^{5}\right)$ such that, for $J^{\prime}=0$, we have $b(s, \theta, E, 0, \eta, H, t)=b_{0}(s, \theta, E, \eta, H, t)$, and such that $b$ satisfies the symmetry condition (B) with $\sin \alpha=-\frac{J^{\prime}-\sqrt{2 H} \sin \alpha_{0}}{E}$. We are now back to our previous notation. The proof Proposition 4.14 (i) goes exactly along the lines of the proof of Proposition 4.6 (see Remark 4.7).

Let us now focus on the proof of (4.31).

If $K(s, \sqrt{2 H}, H, t)$ does not depend on $s$, then $b_{0}(s, \sqrt{2 H}, \theta, \eta, H, t)$ does not depend on $s$, and we can impose that the function $b$ constructed above satisfy equation (D).

Letting $\eta=\frac{J^{\prime}}{h}$, we note that, for $\eta$ in the (compact) support of $b\left(s, \sqrt{2 H}, \theta, J^{\prime}, \eta, H, t\right)$, we have

$$
\alpha-\alpha_{0} \sim \frac{-h \eta}{\sqrt{2 H} \cos \alpha_{0}}(1+O(h))
$$

so that

$$
\frac{-\eta \cos \alpha}{\sqrt{2 H}\left(\alpha-\alpha_{0}\right)} \sim \frac{\cos ^{2} \alpha_{0}}{h}(1+O(h))
$$

We set

$$
\mathcal{Q}_{0}:=\int \operatorname{Tr}\left(-\cos ^{2} \alpha_{0} \partial_{t} K+i\left[-\frac{\partial_{\theta}^{2}}{2}+\cos ^{2} \alpha_{0}\langle V\rangle_{\alpha_{0}}, K\right]_{\omega}\right)(\omega, s, E, H, t)
$$

$$
\rho_{\alpha_{0}}(d \omega, d s, d E, d H, d t)
$$


so that proving (4.31) amounts to showing that $\mathcal{Q}_{0}=0$.

First note that

$$
\begin{aligned}
\mathcal{Q}_{0} & =\int \operatorname{Tr}\left(-\cos ^{2} \alpha_{0} \partial_{t} K+i\left[-\frac{\partial_{\theta}^{2}}{2}+\cos ^{2} \alpha_{0}\langle V\rangle_{\alpha_{0}}, K\right]_{\omega}\right)(\omega, s, \sqrt{2 H}, H, t) \\
& =\int \operatorname{Tr}\left(-\cos ^{2} \alpha_{0} \partial_{t} K+i\left[-\frac{\partial_{\theta}^{2}}{2}+\cos ^{2} \alpha_{0} V, K\right]_{\omega}\right)(\omega \omega, d s, d E, d H, d t) \\
& \rho_{\alpha_{0}}(d \omega, d s, d E, d H, d t)
\end{aligned}
$$

since $\rho_{\alpha_{0}}$ is carried by $E=\sqrt{2 H}$. With a slight abuse of notation we denoted by $V=V(s, E, t)$ the operator of multiplication by $V \circ \Phi\left(s, \theta, E,-\sin \alpha_{0} E, t\right)$ acting on $L^{2}(0,2 \pi)$. Note that it does not depend on $\omega$. It satisfies the condition (4.24) since the function $V \circ \Phi$ satisfies the symmetry condition (B) (since $V$ is only a function of $z$ in the old coordinates). In (4.33) we used the fact that $K(s, \sqrt{2 H}, H, t)$ does not depend on $s$, and the result of Proposition 4.14 (i), to replace $\langle V\rangle_{\alpha_{0}}$ by $V$.

Now, by definition of $\rho_{\alpha_{0}}$, we have

$$
\begin{aligned}
\mathcal{Q}_{0}= & \lim \left\langle n_{h}^{\alpha_{0}},-\cos ^{2} \alpha_{0} \partial_{t} K+i\left[-\frac{\partial_{\theta}^{2}}{2}+\cos ^{2} \alpha_{0} V, K\right]_{\omega}\right\rangle \\
= & \lim \left\langle\chi_{0} \mathscr{V} \mathscr{U} u_{h}\right. \\
& \left.\left(-\cos ^{2} \alpha_{0} \partial_{t} K+i\left[-\frac{\partial_{\theta}^{2}}{2}+\cos ^{2} \alpha_{0} V, K\right]\right)\left(s, h D_{s}, H, h^{2} D_{t}\right) \mathscr{V} \mathscr{U} u_{h}\right\rangle_{L^{2}\left(\mathbb{R}_{s} \times \mathbb{R}_{H}, L^{2}\left(\mathbb{R}_{\theta}\right)\right)}
\end{aligned}
$$

Using the fact that $K(s, E, H, t)=b_{0}\left(s, \theta, E, D_{\theta}, H, t\right)$ and the commutator calculus rule (2.10) for the standard quantization, we obtain

$$
\begin{aligned}
\mathcal{Q}_{0}= & \lim _{h \rightarrow 0^{+}} i\left\langle\chi_{0} \mathscr{V} \mathscr{U} u_{h},\left(\left[\cos ^{2} \alpha_{0} V, K\right]\right)\left(s, h D_{s}, H, h^{2} D_{t}\right) \mathscr{V} \mathscr{U} u_{h}\right\rangle_{L^{2}\left(\mathbb{R}_{s} \times \mathbb{R}_{H}, L^{2}\left(\mathbb{R}_{\theta}\right)\right)} \\
& +\left\langle\chi_{0} \mathscr{V} \mathscr{U} u_{h}, \mathrm{Op}_{h}\left(\left(\eta \partial_{\theta}-i \frac{\partial_{\theta}^{2}}{2}-\cos ^{2} \alpha_{0} \partial_{t}\right) b\left(s, \theta, E, J^{\prime}, \frac{J^{\prime}}{h}, H, h t\right)\right) \mathscr{V} \mathscr{U} u_{h}\right\rangle_{L^{2}\left(\mathbb{R}_{s} \times \mathbb{R}_{\theta} \times \mathbb{R}_{H}\right)}
\end{aligned}
$$

using the notation $\eta=\frac{J^{\prime}}{h}=\frac{J+\sin \alpha_{0} \sqrt{2 H}}{h}$.

We now set

$$
\begin{aligned}
& \mathcal{Q}_{1}:=\lim _{h \rightarrow 0^{+}}\left\langle\chi_{0} \mathscr{V}\right.\left.\mathscr{U} u_{h}, \operatorname{Op}_{h}\left(\left(\eta \partial_{\theta}-\cos ^{2} \alpha_{0} \partial_{t}\right) b\left(s, \theta, E, J^{\prime}, \frac{J^{\prime}}{h}, H, h t\right)\right) \mathscr{V} \mathscr{U} u_{h}\right\rangle_{L^{2}\left(\mathbb{R}_{s} \times \mathbb{R}_{\theta} \times \mathbb{R}_{H}\right)} \\
&+i\left\langle\chi_{0} \mathscr{V} \mathscr{U} u_{h},\left(\left[\cos ^{2} \alpha_{0} V, K\right]\right)\left(s, h D_{s}, H, h^{2} D_{t}\right) \mathscr{V} \mathscr{U} u_{h}\right\rangle_{L^{2}\left(\mathbb{R}_{s} \times \mathbb{R}_{H}, L^{2}\left(\mathbb{R}_{\theta}\right)\right)}
\end{aligned}
$$

so that we have

$$
\mathcal{Q}_{0}=\mathcal{Q}_{1}+\lim _{h \rightarrow 0^{+}}\left\langle\chi_{0} \mathscr{V} \mathscr{U} u_{h}, \mathrm{Op}_{h}\left(-i \frac{\partial_{\theta}^{2}}{2} b\left(s, \theta, E, J^{\prime}, \frac{J^{\prime}}{h}, H, h t\right)\right) \mathscr{V} \mathscr{U} u_{h}\right\rangle_{L^{2}\left(\mathbb{R}_{s} \times \mathbb{R}_{\theta} \times \mathbb{R}_{H}\right)}
$$

Let us for the moment focus on the term $\mathcal{Q}_{1}$, involving only derivatives of order 1 of $b$. As in Remark 4.7, we let $\mathbf{P}(s, \theta, E, J, t, H)=b\left(s, \theta, E, J+\sin \alpha_{0} \sqrt{2 H}, \frac{J+\sin \alpha_{0} \sqrt{2 H}}{h}, H,-t\right)$. Since $b$ is compactly supported in the fifth variable, this is also, modulo $O(h)$,

$$
\mathbf{P}(s, \theta, E, J, t, H)=b\left(s, \theta, E, 0, \frac{J+\sin \alpha_{0} \sqrt{2 H}}{h}, H,-t\right)
$$


Still using the notation $\eta=\frac{J^{\prime}}{h}=\frac{J+\sin \alpha_{0} \sqrt{2 H}}{h}$, we have

$$
\begin{aligned}
\mathcal{Q}_{1}= & \lim \left\langle\mathscr{U} u_{h}, \mathrm{Op}_{h}\left(\left(\eta \partial_{\theta}-\cos ^{2} \alpha_{0} \partial_{t}\right) \mathbf{P}(s, \theta, E, J, t, h H)\right) \mathscr{U} u_{h}\right\rangle_{L^{2}\left(\mathbb{R}_{s} \times \mathbb{T}_{\theta} \times \mathbb{R}_{t}\right)} \\
& +i\left\langle\chi_{0} \mathscr{V} \mathscr{U} u_{h},\left(\left[\cos ^{2} \alpha_{0} V, K\right]\right)\left(s, h D_{s}, H, h^{2} D_{t}\right) \mathscr{V} \mathscr{U} u_{h}\right\rangle_{L^{2}\left(\mathbb{R}_{s} \times \mathbb{R}_{H}, L^{2}\left(\mathbb{R}_{\theta}\right)\right)} \\
= & \lim \left\langle u_{h}, \mathcal{A}_{H}\left(\left(\eta \partial_{\theta}-\cos ^{2} \alpha_{0} \partial_{t}\right) \mathbf{P}\right) u_{h}\right\rangle_{L^{2}\left(\mathbb{R}^{2} \times \mathbb{R}^{2} \times \mathbb{R}_{t}\right)} \\
& +i\left\langle u_{h},\left[\cos ^{2} \alpha_{0} V, \mathcal{A}_{H}(\mathbf{P})\right] u_{h}\right\rangle_{L^{2}\left(\mathbb{R}^{2} \times \mathbb{R}^{2} \times \mathbb{R}_{t}\right)} \\
= & \lim \left\langle u_{h}, \mathcal{A}_{H}\left(-\frac{\eta \cos \alpha}{E\left(\alpha_{0}-\alpha\right)}\left(E \partial_{s}-h \partial_{t}\right) \mathbf{P}\right) u_{h}\right\rangle+i\left\langle u_{h},\left[\cos ^{2} \alpha_{0} V, \mathcal{A}_{H}(\mathbf{P})\right] u_{h}\right\rangle .
\end{aligned}
$$

Using that $b$ (and thus also $\mathbf{P}$ ) satisfies equation (D), together with (4.32), we obtain

$$
\mathcal{Q}_{1}=\lim \frac{\cos ^{2} \alpha_{0}}{h}\left\langle u_{h}, \mathcal{A}_{H}\left(\left(E \partial_{s}-h \partial_{t}\right) \mathbf{P}\right) u_{h}\right\rangle+i\left\langle u_{h},\left[\cos ^{2} \alpha_{0} V, \mathcal{A}_{H}(\mathbf{P})\right] u_{h}\right\rangle
$$

Finally, we use again the Schrödinger equation (4.16) satisfied by $u_{h}$ extended to $\mathbb{R}^{2}$, and rewrite the last line as

$$
\begin{aligned}
\mathcal{Q}_{1} & =\lim \frac{\cos ^{2} \alpha_{0}}{h}\left\langle u_{h},\left[-\frac{i h}{2} \Delta+i h V-h \partial_{t}, \mathcal{A}_{H}(\mathbf{P})\right] u_{h}\right\rangle \\
& =\lim -\frac{\cos ^{2} \alpha_{0}}{h}\left\langle\frac{i h}{2} \frac{\partial u_{h}}{\partial n} \otimes \delta_{\partial \mathbb{D}}, \mathcal{A}_{H}(\mathbf{P}) u_{h}\right\rangle+\lim -\frac{\cos ^{2} \alpha_{0}}{h}\left\langle u_{h}, \mathcal{A}_{H}(\mathbf{P}) \frac{i h}{2} \frac{\partial u_{h}}{\partial n} \otimes \delta_{\partial \mathbb{D}}\right\rangle .
\end{aligned}
$$

Here we need the knowledge of $\mathcal{A}_{H}(\mathbf{P})$ modulo $O\left(h^{2}\right)$ (because of the factor $\frac{\cos ^{2} \alpha_{0}}{h}$ that appears in the previous expression). Our calculations of Proposition A.3 give us the expression

$$
\begin{aligned}
\mathcal{A}_{H}(\mathbf{P})=\mathbf{A}\left(r, u, \sqrt{2 h D_{t}}, h D_{u}, t\right) & +\mathbf{B}\left(r, u, \sqrt{2 h D_{t}}, h D_{u}, t\right) \circ h D_{r} \\
& +i h \mathbf{C}\left(r, u, \sqrt{2 h D_{t}}, h D_{u}, t\right)+i h \mathbf{D}\left(r, u, \sqrt{2 h D_{t}}, h D_{u}, t\right) \circ h D_{r}
\end{aligned}
$$

if $z=(-r \sin u, r \cos u)$ is the decomposition in polar coordinates and $\mathbf{A}, \mathbf{B}, \mathbf{C}, \mathbf{D}$ are the functions associated to $\mathbf{P}$ by the formulas of Proposition A.3.

The terms A, C give a vanishing contribution in formula (4.36) because they are radial operators and $u_{h}$ satisfies a Dirichlet boundary condition. The term $\mathbf{B}$ gives a vanishing condition if $b$ (and hence $\mathbf{P}$ ) satisfy the symmetry condition $(\mathrm{B})$ : in that case we have $\mathbf{B}\left(1, u, \sqrt{2 h D_{t}}, h D_{u}, t\right)=0$. So there just remains to look at the term $\mathbf{D}\left(1, u, \sqrt{2 h D_{t}}, h D_{u}, t\right)$.

Look at formula (A.8) defining the function D. Remember that $\mathbf{P}(s, \theta, \sqrt{2 H}, J, t, H)$ is supported where $J+\sqrt{2 H} \sin \alpha_{0}=O(h)$, so that we have $\partial_{s} \mathbf{P}=O(h)$; also note that, on the set $\left\{J=-\sin \alpha_{0} E\right\}$, the boundary equation $r=1$ amounts to $s= \pm \cos \alpha_{0}, \cos \theta_{1}(r, J, E)= \pm \cos \alpha_{0}$, so that $s \cos \theta_{1}(r, J, E)=\cos ^{2} \alpha_{0}$ in formulas (A.8) and the following lines. We see that the function $\mathbf{D}(1, u, \sqrt{2 H}, J, t)$ coincides, modulo $O(h)$, with $\frac{1}{2 H \cos ^{2} \alpha_{0}} \mathbf{P}^{\sigma}(1, u, \sqrt{2 H}, J, t)$, so that

$$
\begin{aligned}
\mathcal{Q}_{1}= & -\lim \left\langle\frac{h}{2} \frac{\partial u_{h}}{\partial n} \otimes \delta_{\partial \mathbb{D}}, \mathbf{D}\left(1, u, \sqrt{2 h D_{t}}, h D_{u}, t\right) \circ h D_{r} u_{h}\right\rangle \\
& +h \lim \left\langle u_{h}, \mathbf{D}\left(1, u, \sqrt{2 h D_{t}}, h D_{u}, t\right) \circ h D_{r} \frac{h}{2} \frac{\partial u_{h}}{\partial n} \otimes \delta_{\partial \mathbb{D}}\right\rangle \\
= & -\frac{1}{2 \cos ^{2} \alpha_{0}} \lim \left\langle h \frac{\partial u_{h}}{\partial n},\left(E^{-2} \partial_{2}^{2} \mathbf{P}^{\sigma}\right)\left(1, u, \sqrt{2 h D_{t}},-\sqrt{2 h D_{t}} \alpha_{0}, h t, h D_{t}\right) h \frac{\partial u_{h}}{\partial n}\right\rangle .
\end{aligned}
$$

Hence, we obtain

$$
\begin{aligned}
\mathcal{Q}_{1} & =-\lim \frac{\cos ^{2} \alpha_{0}}{h} i h\left\langle\frac{h}{2} \frac{\partial u_{h}}{\partial n} \otimes \delta_{\partial \mathbb{D}}, \mathbf{D}\left(1, u, \sqrt{2 h D_{t}}, h D_{u}, t\right) \circ h D_{r} u_{h}\right\rangle \\
& =-\frac{i}{2} \lim \left\langle h \frac{\partial u_{h}}{\partial n},\left(E^{-2} \partial_{2}^{2} \mathbf{P}\right)\left(1, u, \sqrt{2 h D_{t}},-\sqrt{2 h D_{t}} \alpha_{0}, h t, h D_{t}\right) h \frac{\partial u_{h}}{\partial n}\right\rangle .
\end{aligned}
$$


Using Remark 4.7, this limit expressed in terms of boundary data can also be expressed in terms of the interior, and we see that it equals

$$
\begin{aligned}
\mathcal{Q}_{1} & =\frac{i}{2} \lim \left\langle\mathscr{U} u_{h}, \mathrm{Op}_{h}\left(\partial_{2}^{2} \mathbf{P}\left(s, \theta, E, J^{\prime}, \frac{J^{\prime}}{h}, H, h t\right)\right) \mathscr{U} u_{h}\right\rangle_{L^{2}\left(\mathbb{R}_{s} \times \mathbb{T}_{\theta} \times \mathbb{R}_{t}\right)} \\
& =\frac{i}{2} \lim \left\langle\mathscr{V} \mathscr{U} u_{h}, \mathrm{Op}_{h}\left(\partial_{2}^{2} b\left(s, \theta, E, J^{\prime}, \frac{J^{\prime}}{h}, H, h t\right)\right) \mathscr{V} \mathscr{U} u_{h}\right\rangle_{L^{2}\left(\mathbb{R}_{s} \times \mathbb{T}_{\theta} \times \mathbb{R}_{H}\right)} .
\end{aligned}
$$

Finally coming back to (4.34), this yields $\mathcal{Q}_{0}=0$, that is, identity (4.31). This concludes the proof of Proposition 4.14.

\section{End of the SEmiclassical COnstruction: PRoOF of Theorem 2.4}

In this section, we first prove Proposition 2.8, and then conclude the proof of Theorem 2.4.

5.1. A proof of Proposition 2.8. For $a$ a smooth compactly supported function on $\mathbb{R}^{4}$, we show that

$$
\lim _{h \longrightarrow 0}\left\langle u_{h}, \mathrm{Op}_{h}\left(\partial_{t} a\left(|\xi|^{2}, x \xi_{y}-y \xi_{x}, t, h H\right) u_{h}\right\rangle_{L^{2}\left(\mathbb{R}^{2} \times \mathbb{R}\right)}=0 .\right.
$$

This limit is the same as

$$
\lim _{h \longrightarrow 0}\left\langle u_{h}, \mathrm{Op}_{h}\left(\partial_{t} a\left(2 H, x \xi_{y}-y \xi_{x}, t, h H\right) u_{h}\right\rangle_{L^{2}\left(\mathbb{R}^{2} \times \mathbb{R}\right)}\right.
$$

which is

$$
\lim _{h \rightarrow 0}\left\langle u_{h},\left[\partial_{t}, \operatorname{Op}_{h}\left(a\left(2 H, x \xi_{y}-y \xi_{x}, t, h H\right)\right] u_{h}\right\rangle_{L^{2}\left(\mathbb{R}^{2} \times \mathbb{R}\right)}=\lim _{h \rightarrow 0}\left\langle u_{h},\left[\partial_{t}, a\left(2 h D_{t}, h D_{u}, t, h^{2} D_{t}\right)\right] u_{h}\right\rangle\right.
$$

where $z=(-r \sin u, r \cos u)$ is the decomposition of $z=(x, y)$ into polar coordinates. Because of the equation satisfied by $u_{h}$, this is also (with $\Delta_{D}$ the Dirichlet laplacian)

$$
\lim _{h \longrightarrow 0}\left\langle u_{h},\left[-i \frac{\Delta_{D}}{2}+i V, a\left(2 h D_{t}, h D_{u}, t, h^{2} D_{t}\right)\right] u_{h}\right\rangle
$$

Note that $a\left(2 h D_{t}, h D_{u}, t, h^{2} D_{t}\right)$ actually defines an operator on $L^{2}(\mathbb{D})$ as it is tangential to $\partial \mathbb{D}$. This limit vanishes, because $\Delta_{D}$ commutes with $a\left(2 h D_{t}, h D_{u}, t, h^{2} D_{t}\right)$ and because

$$
\left[V, a\left(2 h D_{t}, h D_{u}, t, h^{2} D_{t}\right)\right]=O(h) .
$$

This concludes the proof of Proposition 2.8.

5.2. End of the proof of Theorem 2.4. We are now in a position to prove Theorem 2.4.

The measure $\ell_{\alpha_{0}}$ and the function $\sigma_{\alpha_{0}}$ of Theorem 2.4 (iii) are the ones appearing in Remark 4.16. The object called $\nu_{L e b}$ in Theorem 2.4 (ii) is defined as

$$
\left.\nu_{L e b}=\mu_{s c}\right\rceil_{\alpha \notin \pi \mathbb{Q}}+\sum_{\alpha_{0} \in \pi \mathbb{Q}} m^{\alpha_{0}}
$$

where $m^{\alpha_{0}}$ was defined in Remark 4.5. For $\alpha \notin \pi \mathbb{Q}$, we must have

$$
\left.\mu_{s c}\right\rceil_{\mathcal{I}_{\alpha}}(t)=\int c_{1}(t, E, J) \lambda_{E, J} d \nu_{1}(E, J)
$$

for some nonnegative measure $\nu_{1}$ (carried by $\{J=-\sin \alpha E\}$ ) and some measurable function $c_{1}(t, E, J)$. But, because the image of $\mu_{s c}$ under the map $M:(z, \xi) \mapsto(E, J)$ does not depend on $t$ (see below), the function $c_{1}(t, E, J)$ actually does not depend on $t$.

The two invariance properties Proposition 4.6 (invariance w.r.t. $s$ ) and Theorem 4.8 (i) (invariance w.r.t. $\theta$ ) also imply that $m^{\alpha_{0}}$ is of the form

$$
m^{\alpha_{0}}(t)=\int c_{2}(t, E, J) \lambda_{E, J} d \nu_{2}(E, J)
$$

for some nonnegative measure $\nu_{2}$ (carried by $\left.\left\{J=-\sin \alpha_{0} E\right\}\right)$. 
We now prove that the function $c_{2}(t, E, J)$ actually does not depend on $t$. For this, we remark that the same proof as that of Proposition 2.8 above applies if we replace

$$
\mathrm{Op}_{h}\left(\partial_{t} a\left(|\xi|^{2}, x \xi_{y}-y \xi_{x}, t, h H\right)\right)
$$

in the first line by

$$
\mathscr{U}^{*} \operatorname{Op}_{h}\left(\partial_{t} a\left(E^{2}, J, t, h H\right)(1-\chi)\left(\frac{J+\sin \alpha_{0} \sqrt{2 H}}{h R}\right)\right) \mathscr{U}
$$

in the limits $h \longrightarrow 0$ followed by $R \longrightarrow+\infty$.

Using the notation of Remark 4.5, this shows that the image of $m^{\alpha_{0}}$ under the map $M$ is independent of $t$. Since we already know that $m^{\alpha_{0}}(t)$ is of the form $\int c_{2}(t, E, J) \lambda_{E, J} d \nu_{2}(E, J)$ for some nonnegative measure $\nu_{2}$, we conclude that $c_{2}(t, E, J)$ actually does not depend on $t$.

The proof of Theorem 2.4 is now complete.

\section{The microlocal Construction: Sketch of the Proof of Theorem 2.6}

Herein we use the definitions and notation introduced in Sections 2.1 and 2.5.

Let $\left(u_{n}^{0}\right)$ be a sequence of initial data, normalized in $L^{2}$, and as above denote $u_{n}(z, t)=$ $U_{V}(t) u_{n}^{0}(z)$ or in short $u_{n}=U_{V} u_{n}^{0}$.

6.1. Structure of $\mu_{c}$. Let $a \in \mathcal{S}_{0}$. Recall that $\left\langle\mu_{c}, a\right\rangle$ is defined as the limit (after extraction of subsequences) as $n \longrightarrow+\infty$ followed by $R \longrightarrow+\infty$ of

$$
\left\langle W_{c, n, R}, a\right\rangle:=\left\langle u_{n}, \mathrm{Op}_{1}\left(\chi\left(\frac{|\xi|^{2}+|H|}{R^{2}}\right) a(z, \xi, t, H)\right) u_{n}\right\rangle_{L^{2}\left(\mathbb{R}^{2} \times \mathbb{R}\right)} .
$$

On the support of $\chi\left(\frac{|\xi|^{2}+|H|}{R^{2}}\right) a(z, \xi, t, H)$ note that $|H| \leq K R^{2}$ if $\chi$ is supported in $[-K, K]$.

Let $g$ be a smooth compactly supported function on $\mathbb{R}$, taking the value 1 on $[-K, K]$. For $R>0$ define the operator $P_{R}: L^{2}(\mathbb{D}) \mapsto L^{2}(\mathbb{D})$ by

$$
\left.P_{R} u=g\left(\frac{D_{t}}{R^{2}}\right) U_{V}(t) u\right\rceil_{t=0} .
$$

By the results of Appendix C (in particular Remark C.3), we know that $P_{R}$ has the following properties:

- For fixed $R, P_{R}$ is compact, and we have $\left\|\nabla P_{R} u\right\|_{L^{2}(\mathbb{D})} \leq C R\|u\|_{L^{2}(\mathbb{D})}$ for some constant $C$ (that depends on the function $g$, but not on $R$ ).

- For any $u$, we have $P_{R} u \longrightarrow u$ in $L^{2}(\mathbb{D})$ as $R \longrightarrow+\infty$.

- $\left\langle\mu_{c}, a\right\rangle$ is the limit as $n \longrightarrow+\infty$ followed by $R \longrightarrow+\infty$ of

$$
\left\langle u_{n}, \mathrm{Op}_{1}\left(\chi\left(\frac{|\xi|^{2}+|H|}{R^{2}}\right) a(z, \xi, t, H)\right) U_{V} P_{R} u_{n}^{0}\right\rangle_{L^{2}\left(\mathbb{R}^{2} \times \mathbb{R}\right)} .
$$

Let $K_{t}\left(z, z^{\prime}\right)$ be the kernel of $U_{V}(t)$. Let $B_{R}$ be the bounded operator on $L^{2}(\mathbb{D})$ with kernel

$$
\begin{aligned}
B_{R}\left(z_{1}, z_{2}\right)=\frac{1}{(2 \pi)^{3}} \int \overline{K_{t}\left(z, z_{1}\right)} \mathbb{1}_{\mathbb{D}}(z) a(z, \xi, t, H) \chi\left(\frac{|\xi|^{2}+|H|}{R^{2}}\right) \\
e^{i \xi\left(z-z^{\prime}\right)} e^{i H\left(t-t^{\prime}\right)} \mathbb{1}_{\mathbb{D}}\left(z^{\prime}\right) K_{t^{\prime}}\left(z^{\prime}, z_{2}\right) d z d z^{\prime} d t d t^{\prime} d H d \xi
\end{aligned}
$$

so that (6.1) is $\left\langle u_{n}^{0}, B_{R} u_{n}^{0}\right\rangle_{L^{2}(\mathbb{D})}$ and (6.2) is $\left\langle u_{n}^{0}, B_{R} P_{R} u_{n}^{0}\right\rangle_{L^{2}(\mathbb{D})}$.

Call $\rho_{0}$ a weak-* limit of the sequence of trace class operators $\left|u_{n}^{0}\right\rangle\left\langle u_{n}^{0}\right|$ on $L^{2}(\mathbb{D})$. Then for fixed $R\left\langle W_{c, n, R}, a\right\rangle$ converges to $\operatorname{Tr}_{L^{2}(\mathbb{D})}\left(B_{R} P_{R} \rho_{0}\right)$. Letting now $R \longrightarrow+\infty$ we find the expression

$$
\left\langle\mu_{c}, a\right\rangle=\operatorname{Tr}_{L^{2}(\mathbb{D})}\left(B_{\infty} \rho_{0}\right)
$$

where $B_{\infty}$ is defined as in (6.3) with $\chi\left(\frac{|\xi|^{2}+|H|}{\infty}\right)=1$. The expression of $B_{\infty}$ is simpler when $a$ does not depend on $H$, in this case we have

$$
B_{\infty}=\int U_{V}(t)^{*} \mathbb{1}_{\mathbb{D}} a\left(z, D_{z}, t\right) \mathbb{1}_{\mathbb{D}} U_{V}(t) d t .
$$


In particular if $a$ does not depend on $H$ we have

$$
\left\langle\mu_{c}, a\right\rangle=\operatorname{Tr}_{L^{2}(\mathbb{D})}\left(\int U_{V}(t)^{*} \mathbb{1}_{\mathbb{D}} a\left(z, D_{z}, t\right) \mathbb{1}_{\mathbb{D}} U_{V}(t) \rho_{0} d t\right)
$$

and more generally we write (in a somewhat fuzzy notation)

$$
\left\langle\mu_{c}, a\right\rangle=\operatorname{Tr}_{L^{2}(\mathbb{D})}\left(\int U_{V}(t)^{*} \mathbb{1}_{\mathbb{D}} a\left(x, D_{z}, t, D_{t}\right) \mathbb{1}_{\mathbb{D}} U_{V}(t) \rho_{0} d t\right)
$$

to mean the well-defined expression (6.4).

6.2. Structure of $\mu^{\infty}$ and proof of Theorem 2.6. Let $\eta=\frac{\xi}{\sqrt{2 H}}$. On the support of $\mu^{\infty}, \eta$ has norm 1. To any pair $(z, \eta) \in \mathbb{D} \times \mathbb{S}^{1}$ we now associate $j=x \eta_{y}-y \eta_{x}$ and $\alpha=-\arcsin j$ which is the angle that the billiard ray issued from $(z, \eta)$ makes with the inner normal when it bounces on the boundary of the disk. Exactly as in Lemma 3.2 we decompose $\mu^{\infty}$ as a sum of nonnegative measures:

$$
\left.\left.\mu^{\infty}=\mu^{\infty}\right\rceil_{\alpha \notin \pi \mathbb{Q}}+\sum_{r \in \mathbb{Q} \cap[-1 / 2,1 / 2]} \mu^{\infty}\right\rceil_{\alpha=r \pi} .
$$

The invariance (2.17) implies that $\left.\mu^{\infty}\right\rceil_{\alpha \notin \pi \mathbb{Q}}$ is of the form $\int_{E>0,|J| \leq E, \alpha \notin \pi \mathbb{Q}} \lambda_{E, J} d \bar{\mu}^{\infty}(E, J)$ The fact that $\bar{\mu}^{\infty}$ does not depend on $t$ is the microlocal version of Proposition 2.8.

We now fix $r_{0} \in \mathbb{Q} \cap(-1 / 2,1 / 2)$, write $\alpha_{0}=r_{0} \pi$ and wish to study $\left.\mu^{\infty}\right\rceil_{\alpha=\alpha_{0}}$. We define

$$
\begin{aligned}
& \left\langle\mu_{\alpha_{0}}^{\infty}, a\right\rangle \\
& :=\lim _{R^{\prime}} \lim _{R \rightarrow \infty} \lim _{n \rightarrow+\infty} \\
& \left\langle u_{n}, \mathrm{Op}_{1}\left(\left(1-\chi\left(\frac{|\xi|^{2}+H}{R^{2}}\right)\right) a(z, \xi, t, H) \chi\left(\frac{J+\sin \alpha_{0} \sqrt{2 H}}{R^{\prime}}\right)\right) u_{n}\right\rangle_{L^{2}\left(\mathbb{R}^{2} \times \mathbb{R}\right)} \\
& =\lim _{R^{\prime}} \lim _{R} \lim _{n} \\
& \left\langle\mathscr{U} u_{n}, \mathrm{Op}_{1}\left(\left(1-\chi\left(\frac{H}{R^{2}}\right)\right) a \circ \Phi(s, \theta, \sqrt{2 H}, J, t, H) \chi\left(\frac{J+\sin \alpha_{0} \sqrt{2 H}}{R^{\prime}}\right)\right) \mathscr{U} u_{n}\right\rangle_{L^{2}(\mathbb{R} \times \mathbb{T} \times \mathbb{R})}
\end{aligned}
$$

and

$$
\begin{aligned}
& \left\langle\mu^{\infty, \alpha_{0}}, a\right\rangle \\
& :=\lim _{R^{\prime}} \lim _{R \rightarrow \infty} \lim _{n \rightarrow+\infty} \\
& \left\langle u_{n}, \mathrm{Op}_{1}\left(\left(1-\chi\left(\frac{|\xi|^{2}+H}{R^{2}}\right)\right) a(z, \xi, t, H)\left(1-\chi\left(\frac{J+\sin \alpha_{0} \sqrt{2 H}}{R^{\prime}}\right)\right)\right) u_{n}\right\rangle_{L^{2}\left(\mathbb{R}^{2} \times \mathbb{R}\right)} \\
& =\lim _{R^{\prime}} \lim _{R} \lim _{n} \\
& \left\langle\mathscr{U} u_{n}, \mathrm{Op}_{1}\left(\left(1-\chi\left(\frac{H}{R^{2}}\right)\right) a \circ \Phi(s, \theta, \sqrt{2 H}, J, t, H)\left(1-\chi\left(\frac{J+\sin \alpha_{0} \sqrt{2 H}}{R^{\prime}}\right)\right)\right) \mathscr{U} u_{n}\right\rangle .
\end{aligned}
$$

The following theorem is proven in essentially the same way as in the semiclassical case:

Theorem 6.1. (i) $\mu^{\infty, \alpha_{0}}$ is invariant under rotations (that is, under the flow of $P_{1}$ ). It is a multiple of the Lebesgue measure $\lambda_{\left(1,-\sin \alpha_{0}\right)}$. That is, $\mu^{\infty, \alpha_{0}}=c\left(\alpha_{0}\right) \lambda_{\left(1,-\sin \alpha_{0}\right)}$ with $c\left(\alpha_{0}\right) \geq 0$ independent of $t$.

(ii) for every $\alpha_{0} \in \pi \mathbb{Q} \cap(-\pi / 2, \pi / 2)$, we can build from the sequence of initial conditions $\left(u_{n}\right)$ a nonnegative measure $\sigma_{\alpha_{0}}(d \omega, d s, d E, d H, d t)$ (carried by $\left\{H=E^{2} / 2\right\}$ ) on $\mathbb{R} / 2 \pi \mathbb{Z} \times \mathbb{R}_{s} \times \mathbb{S}_{E, H}^{2} \times \mathbb{R}_{t}$, taking values in the trace-class operators on $L^{2}(0,2 \pi)$, so that $\mu_{\alpha_{0}}^{\infty}$ is the measure carried by the 
set $\left\{j=-\sin \alpha_{0}\right\} \cap\left\{H=E^{2} / 2\right\}$ such that

$$
\begin{aligned}
& \int a_{\mathrm{hom}}(z, \xi, t) \mu_{\alpha_{0}}^{\infty}(d z, d \xi, d t, d H) \\
& =\operatorname{Tr}_{L^{2}(0,2 \pi)}\left(\int m_{a_{\mathrm{hom}} \circ \Phi}\left(s, \cdot, 1,-\sin \alpha_{0}, t\right) \sigma_{\alpha_{0}}(d \omega, d s, d E, d H, d t)\right) .
\end{aligned}
$$

If in addition a is symmetric w.r.t. the boundary, we have

$$
\begin{aligned}
& \int a_{\mathrm{hom}}(z, \xi) \mu_{\alpha_{0}}^{\infty}(d z, d \xi, t, d H) \\
& =\operatorname{Tr}_{L^{2}(0,2 \pi)}\left(\int U_{\alpha_{0}, \omega}(t)^{*} m_{\left\langle a_{\mathrm{hom}}\right\rangle_{\alpha_{0}} \circ \Phi}\left(\cdot, 1,-\sin \alpha_{0}\right) U_{\alpha_{0}, \omega}(t) \sigma_{\alpha_{0}}(d \omega, d s, d E, d H, 0)\right) .
\end{aligned}
$$

The decomposition forlmula of Theorem 2.6 (i) now holds with

- the distribution $\mu_{c} \in \mathcal{S}_{0}^{\prime}$ described in Section 6.1;

- the measure $\mu_{L e b}$ given by

$$
\left.\mu_{L e b}=\mu^{\infty}\right\rceil_{\alpha \notin \pi \mathbb{Q}}+\sum_{\alpha_{0} \in \pi \mathbb{Q} \cap(-\pi / 2, \pi / 2)} \mu^{\infty, \alpha_{0}} ;
$$

- for $\alpha_{0} \in \pi \mathbb{Q} \cap(-\pi / 2, \pi / 2)$ the measure $\mu_{\alpha_{0}}$ given by

$$
\mu_{\alpha_{0}}(t)=\mu_{\alpha_{0}}^{\infty}(t)
$$

- for $\alpha_{0}= \pm \pi / 2$ the measure $\mu_{\alpha_{0}}$ given by

$$
\left.\mu_{\alpha_{0}}=\mu^{\infty}\right\rceil_{\alpha=\alpha_{0}} .
$$

Theorem 6.1 then implies Theorem 2.6.

\section{Proof of Theorems 1.2 And 1.3: Observability inequalities}

In this section, we prove Theorems 1.2 and 1.3 using the microlocal version of our results. We could have chosen to do it with semiclassical measures as well. However, since there is no natural frequency-scale, it would have required to perform a dyadic decomposition in frequency (see for instance [Leb92, AM14]). Note that the idea of proving observability inequalities using microlocal defect measures is due to Lebeau [Leb96].

7.1. Unique continuation for microlocal measures. The goal of this section is to prove a unique continuation result for microlocal measures $\mu_{m l}$ associated to solutions of the Schrödinger equation (1.1). According to Theorem 2.6, such a measure decomposes as

$$
\mu_{m l}=\mu^{\infty}+\mu_{c}
$$

that we shall study independently.

In order to state the result for $\mu^{\infty}$, we introduce the following notation. For $z \in \partial \mathbb{D}$, we define

$$
S_{z}^{+}=\left\{\xi \in \mathbb{R}^{2}, \xi \cdot z>0\right\}, \quad \bar{S}_{z}^{+}=\left\{\xi \in \mathbb{R}^{2}, \xi \cdot z \geq 0\right\} .
$$

The set $S^{+}$defined in Section 2.2 is $S^{+}=\bigcup_{z \in \partial \mathbb{D}} S_{z}^{+}$and

$$
\bigcup_{z \in \partial \mathbb{D}} \bar{S}_{z}^{+}=\left\{\Phi\left(\left(1-(J / E)^{2}\right)^{\frac{1}{2}}, \theta, E, J\right), E>0,|J| \leq E, \theta \in \mathbb{R} / 2 \pi \mathbb{Z}\right\} .
$$

The following two lemmas are respectively useful for the proof of internal and boundary observability.

Lemma 7.1. Fix $T>0$. Take $b \in \mathcal{S}^{0}$ independent of $(t, H)$ and assume that

$$
\text { there exists } z_{0} \in \partial \mathbb{D} \text { such that } b>0 \text { in a neighbourhood of } \bar{S}_{z_{0}}^{+} \text {. }
$$

$$
\text { Then } \int_{0}^{T} \int_{\mathbb{R}^{2} \times \mathbb{S}_{H, \xi}^{2}} b_{\text {hom }}^{2}(z, \xi) \mu^{\infty}(d z, d H, d \xi, t) d t=0 \text { implies } \mu^{\infty}=0 \text { on } \mathbb{R}_{t} \times \mathbb{R}_{z}^{2} \times \mathbb{S}_{H, \xi}^{2} \text {. }
$$


Lemma 7.2. Take any nonempty set $\Gamma \subset \partial \mathbb{D}$ and $T>0$. Then $\mu_{m l}^{\partial}=0$ on $T^{*}((0, T) \times \Gamma)$ implies $\mu^{\infty}=0$ on $\mathbb{R}_{t} \times \mathbb{R}_{z}^{2} \times \mathbb{S}_{H, \xi}^{2}$.

The proof of these lemmas relies on the properties of $\mu^{\infty}$ together with a unique continuation result for the one dimensional Schrödinger flows $U_{\alpha_{0}, \omega}(t)$ on $L^{2}(0,2 \pi)$ from any nonempty open set $(0, T) \times \Omega$, where $\Omega \subset(0,2 \pi)$. Such unique continuation property holds as soon as $\langle V\rangle_{\alpha_{0}} \in$ $L^{\infty}((0, T) \times(0,2 \pi))$, for instance as a consequence of [Lau10, Appendix B] (see also the references therein).

Concerning $\mu_{c}$ we have the following result.

Lemma 7.3. Let $\Omega \subset \mathbb{D}$ be a nonempty open set. Assume that the unique continuation property $\left(\mathrm{UCP}_{V, \Omega, T}\right)$ holds. Then we have

$$
\left\langle\mu_{c}, \mathbb{1}_{(0, T) \times \Omega}\right\rangle=0 \Longrightarrow \mu_{c}=0 .
$$

The unique continuation property $\left(\mathrm{UCP}_{V, \Omega, T}\right)$ is for instance known to hold (in any time $T>0$ and for any nonempty open set $\Omega$ ) if $V$ is analytic in $(t, z)$ as a consequence of the Holmgren theorem (as stated by Hörmander [Hör76, Theorem 5.3.1]). If $V=V(z)$ is smooth and does not depend on $t$, it is proved in the next section. Note that this last result can be extended to the case where $V$ is continuous outside a set of zero measure zero, see [AM14].

Remark 7.4. Note that the analogues of the unique continuation results of Lemmas 7.1, 7.2 and 7.3 also hold for semiclassical measures. We chose not to state them here for the sake of brevity.

Proof of Lemma \%.1. We decompose $\mu^{\infty}$ as in Theorem 2.6

$$
\mu^{\infty}(t, \cdot)=\mu_{L e b}+\sum_{\alpha_{0} \in \pi \mathbb{Q} \cap[-\pi / 2, \pi / 2]} \mu_{\alpha_{0}}(t, \cdot) .
$$

As every term in this sum is a non-negative measure, the assumption on $\mu^{\infty}$ implies

$$
\begin{aligned}
& \int_{0}^{T} \int_{\mathbb{R}^{2} \times \mathbb{S}^{2}} b_{\mathrm{hom}}^{2}(z, \xi) \mu_{L e b}(d z, d H, d \xi, t) d t=0, \\
& \int_{0}^{T} \int_{\mathbb{R}^{2} \times \mathbb{S}^{2}} b_{\mathrm{hom}}^{2}(z, \xi) \mu_{\alpha_{0}}(d z, d H, d \xi, t) d t=0,
\end{aligned}
$$

for all $\alpha_{0} \in \pi \mathbb{Q} \cap[-\pi / 2, \pi / 2]$.

Still according to Theorem 2.6, $\mu_{L e b}$ is of the form $\int_{E>0,|J| \leq E} \lambda_{E, J} d \mu^{\prime}(E, J)$ for some nonnegative measure $\mu^{\prime}$ on $\mathbb{R} P^{1}$. Together with (7.2), this reads

$$
0=\int_{E>0,|J| \leq E} \int_{T(E, J)} b_{\mathrm{hom}}^{2} \circ \Phi(s, \theta, E, J) \lambda_{E, J}(d s, d \theta) \mu^{\prime}(d E, d J) .
$$

Recall (see Section 2.2) that $\lambda_{E, J}(d s, d \theta)=c(E, J) d s d \theta$ where $c(E, J)=\left(\int_{T(E, J)} d s d \theta\right)^{-1}>0$, so that we have

$$
0=\int_{E>0,|J| \leq E}\left(\int_{T(E, J)} b_{\mathrm{hom}}^{2} \circ \Phi(s, \theta, E, J) d s d \theta\right) c(E, J) \mu^{\prime}(d E, d J) .
$$

Now, for any $(E, J)$ such that $E>0,|J| \leq E$, there exists $\theta \in \mathbb{S}^{1}$ (depending only on $J / E$ ), such that

$$
\Phi\left(\left(1-(J / E)^{2}\right)^{\frac{1}{2}}, \theta, E, J\right) \in \bar{S}_{z_{0}}^{+} .
$$

Assumption (7.1) then implies that $\int_{T(E, J)} b_{\text {hom }}^{2} \circ \Phi(s, \theta, E, J) d s d \theta>0$ for any $(E, J)$. As a consequence, $\mu_{L e b}$ vanishes identically.

Let us now consider $\alpha_{0}= \pm \pi / 2$. The rotation invariance given by Theorem 2.6 together with Assumption (7.1) imply that $\mu_{ \pm \pi / 2}$ vanish. 
Let us now consider $\alpha_{0} \in \pi \mathbb{Q} \cap(-\pi / 2, \pi / 2)$. The measure $\mu_{\alpha_{0}}$ is supported by $\mathcal{I}_{\alpha_{0}}$ and invariant by the billiard flow, so that

$$
\int_{0}^{T} \int_{\mathbb{R}^{2} \times \mathbb{S}^{2}} b_{\mathrm{hom}}^{2} \mu_{\alpha_{0}}(d z, d H, d \xi, t) d t=\int_{0}^{T} \int_{\mathbb{R}^{2} \times \mathbb{S}^{2}}\left\langle b_{\mathrm{hom}}^{2}\right\rangle_{\alpha_{0}} \mu_{\alpha_{0}}(d z, d H, d \xi, t) d t
$$

Using Theorem 2.6 with (7.3), we obtain

$$
0=\int \operatorname{Tr}_{L^{2}(0,2 \pi)}\left(B_{\alpha_{0}} \sigma_{\alpha_{0}}\right) d \ell_{\alpha_{0}} d t, \quad \text { with } B_{\alpha_{0}}:=m_{\left\langle b_{\text {hom }}^{2}\right\rangle_{\alpha_{0}}}^{\alpha_{0}} .
$$

According to Corollary 4.17, this yields

$$
0=\int \operatorname{Tr}_{L^{2}(0,2 \pi)}\left(B_{\alpha_{0}} U_{\alpha_{0}, \omega}(t) \sigma_{\alpha_{0}}(\omega, E, H, 0) U_{\alpha_{0}, \omega}^{*}(t)\right) d \ell_{\alpha_{0}} d t .
$$

Since the integrand is non-negative, we have for $\ell_{\alpha_{0}}$-almost every $(\omega, E, H)$,

$$
0=\int_{0}^{T} \operatorname{Tr}_{L^{2}(0,2 \pi)}\left(B_{\alpha_{0}} U_{\alpha_{0}, \omega}(t) \sigma_{\alpha_{0}}(\omega, E, H, 0) U_{\alpha_{0}, \omega}^{*}(t)\right) d t .
$$

For $\ell_{\alpha_{0}}$-almost every $(\omega, E, H), \sigma_{\alpha_{0}}(\omega, E, H, 0)$ is a non-negative trace-class operator. We can decompose it as a sum of of orthogonal projectors on its eigenfunctions:

$$
\sigma_{\alpha_{0}}(\cdot, 0)=\sum_{k \in \mathbb{N}} \lambda_{k}\left|\varphi_{k}\right\rangle\left\langle\varphi_{k}\right|, \quad \text { with } \quad \lambda_{k} \geq 0, \quad \sum_{k \in \mathbb{N}} \lambda_{k}=1, \quad\left\langle\varphi_{k} \mid \varphi_{j}\right\rangle_{L^{2}(0,2 \pi)}=\delta_{k j} .
$$

Note that $\lambda_{k}, \varphi_{k}$ depend on $(\omega, E, H)$. Now Equation (7.4) is equivalent to having, for all $k \in \mathbb{N}$, such that $\lambda_{k}>0$,

$$
0=\int_{0}^{T} \int_{0}^{2 \pi}\left\langle b_{\mathrm{hom}}^{2}\right\rangle_{\alpha_{0}} \circ \Phi\left(s, \theta, E,-E \sin \alpha_{0}\right)\left|U_{\alpha_{0}, \omega}(t) \varphi_{k}\right|^{2}(\theta) d \theta d t .
$$

As above, there exists $\theta \in \mathbb{S}^{1}$ depending only on $\alpha_{0}$, such that

$$
\Phi\left(\cos \alpha_{0}, \theta, E,-E \sin \alpha_{0}\right) \in \bar{S}_{z_{0}}^{+} .
$$

Hence, $\left\langle b_{\text {hom }}^{2}\right\rangle_{\alpha_{0}}>0$ in a neighborhood of this $\theta$. Then (7.5) implies that $U_{\alpha_{0}, \omega}(t) \varphi_{k}$ vanishes in a nonempty open subset of $(0, T) \times(0,2 \pi)$. One dimensional unique continuation (see e.g. [Lau10, Appendix B] and the references therein) then implies that $\varphi_{k}=0$. Therefore, $\sigma_{\alpha_{0}}(\omega, E, H, 0)$ vanishes $\ell_{\alpha_{0}}$-almost everywhere, which yields $\mu_{\alpha_{0}}=0$.

This finally proves that $\mu^{\infty}=0$ and concludes the proof of the lemma.

Proof of Lemma 7.2. Let us fix $z_{0} \in \Gamma$ and prove that $\mu^{\infty}$ vanishes in a neighborhood of $\bar{S}_{z_{0}}^{+}$. The result shall then follow from Lemma 7.1. First, according to (2.26), the assumption implies that $\left.\mu^{\infty}\right\rceil_{T^{*}((0, T) \times \Gamma)}$ vanishes. Second, as a consequence of the assumption together with (2.25), we have $\left.\mu_{m l}^{S}\right\rceil_{(z, \xi) \in S^{+}, z \in \Gamma}=0$. Coming back to the definition of the measure $\mu_{m l}^{S}$ in Section 2.8.1, this implies that $\mu^{\infty}$ vanishes on all trajectories of the billiard flow touching the boundary on $\left\{(z, \xi) \in S^{+}, z \in \Gamma\right\}$. In particular, this yields $\left.\mu^{\infty}\right\rceil \frac{}{S_{z_{0}}^{+}}$and the result follows from Lemma 7.1.

Proof of Lemma 7.3. Theorem 2.6 (and Theorem 2.7) together with $\left\langle\mu_{c}, \mathbb{1}_{(0, T) \times \Omega}\right\rangle=0$ imply that

$$
0=\operatorname{Tr}_{L^{2}(\mathbb{D})}\left(\int_{0}^{T} U_{V}(t)^{*} m_{\mathbb{1}_{\Omega}} U_{V}(t) \rho_{0} d t\right)
$$

where $m_{\mathbb{1}_{\Omega}}$ is the multiplication operator in $L^{2}(\mathbb{D})$ by the function $\mathbb{1}_{\Omega}$. As in the proof of Lemma 7.1, this implies that for any eigenfunction $\varphi$ of $\rho_{0}$, we have

$$
0=\int_{0}^{T} \int_{\Omega}\left|U_{V}(t) \varphi\right|^{2}(z) d z d t
$$

The unique continuation property $\left(\mathrm{UCP}_{V, \Omega, T}\right)$ then implies $\varphi=0$. This proves that $\rho_{0}=0$ which concludes the proof of the lemma.

\subsection{Interior observability inequality: proof of Theorem 1.2.}


7.2.1. Unique continuation implies observability. In this section, we prove the observability inequality (1.5) assuming that $\left(\mathrm{UCP}_{V, \Omega, T}\right)$ holds. Instead of proving (1.5) for any open set $\Omega \subset \mathbb{D}$ containing a neighbourhood in $\mathbb{D}$ of a point of $\partial \mathbb{D}$, we prove the equivalent statement: for any function $b \in C^{0}\left(\mathbb{R}^{2}\right.$ ) (also considered as a function in $C^{0}(\overline{\mathbb{D}})$ ) which is positive on a nonempty open subset of $\partial \mathbb{D}$, for any $T>0$, there exists $C>0$ such that the following inequality holds:

$$
\left\|u^{0}\right\|_{L^{2}(\mathbb{D})}^{2} \leq C \int_{0}^{T}\left\|b(z) U_{V}(t) u^{0}\right\|_{L^{2}(\mathbb{D})}^{2} d t .
$$

Note that under these conditions on $\Omega$ and $b$, inequalities (7.9) and (7.6) are equivalent.

We proceed by contradiction and suppose that the observability inequality (7.6) is not satisfied. Thus, there exists a sequence $\left(u_{n}^{0}\right)_{n \in \mathbb{N}}$ in $L^{2}(\mathbb{D})$ such that

$$
\begin{aligned}
& \left\|u_{n}^{0}\right\|_{L^{2}(\mathbb{D})}=1, \\
& \int_{0}^{T}\left\|b(z) U_{V}(t) u_{n}^{0}\right\|_{L^{2}(\mathbb{D})}^{2} d t \rightarrow 0, \quad n \rightarrow \infty,
\end{aligned}
$$

We write $u_{n}(t)=U_{V}(t) u_{n}^{0}$ the associated solution of (1.1)-(1.2). As in Section 1, we extend $u_{n}$ to $\mathbb{R}^{2}$ by zero outside $\mathbb{D}$ (and still use the notation $u_{n}$ for its extension).

After having extracted a subsequence, we associate to $\left(u_{n}\right)$ a microlocal measure

$$
\mu_{m l}=\mu^{\infty}+\mu_{c}
$$

as in Theorem 2.6. Equation (7.8) implies that

$$
\int_{0}^{T} \int_{\mathbb{R}^{2} \times \mathbb{S}^{2}} b^{2}(z) \mu^{\infty}(d z, d H, d \xi, t) d t=0, \quad\left\langle\mu_{c}, \mathbb{1}_{(0, T)} \otimes b^{2}\right\rangle=0 .
$$

Lemmas 7.1 and 7.3 imply that $\mu^{\infty}=0$ and $\mu_{c}=0$ respectively. However, equation (7.7) implies that

$$
\left\langle\mu_{m l}, \mathbb{1}_{(0, T)} \otimes 1\right\rangle=T .
$$

This yields a contradiction and concludes the proof. Note that $\left(\mathrm{UCP}_{V, \Omega, T}\right)$ has only be used to apply Lemma 7.3 in order to get rid of the term $\mu_{c}$.

7.2.2. Observability for time independent potentials. The structure of the proof in this setting is classical [BLR92, Leb92]. In a first step, we prove the following weakened observability inequality:

$$
\left\|u^{0}\right\|_{L^{2}(\mathbb{D})}^{2} \leq C \int_{0}^{T}\left\|U_{V}(t) u^{0}\right\|_{L^{2}(\Omega)}^{2} d t+C\left\|u^{0}\right\|_{H^{-1}(\mathbb{D})}^{2},
$$

In a second step, we conclude the proof of Theorem 1.2 using a unique continuation property for eigenfunctions of the elliptic operator $-\Delta_{D}+V$.

The first step is similar to Section 7.2.1. We consider a sequence of initial data $\left(u_{n}^{0}\right)$ contradicting (7.9). It satisfies (7.7), (7.8), together with

$$
\left\|u_{n}^{0}\right\|_{H^{-1}(\mathbb{D})} \rightarrow 0, \quad n \rightarrow \infty .
$$

As before, we consider the associated microlocal measure $\mu_{m l}=\mu^{\infty}+\mu_{c}$. Note now that $(7.10)$ implies that $\mu_{c}=0$. The rest of the proof is completely similar.

We now prove that (7.9) implies the observability inequality (1.5): this step is by now classical [BLR92, Leb92] but we include it for the sake of completeness. We proceed again by contradiction and suppose that the inequality

$$
\left\|u^{0}\right\|_{H^{-1}(\mathbb{D})} \leq C \int_{0}^{T}\left\|b(z) U_{V}(t) u^{0}\right\|_{L^{2}(\Omega)}^{2} d t
$$

is not satisfied. Then, there exists a sequence $\left(u_{n}^{0}\right)_{n \in \mathbb{N}}$ in $L^{2}(\mathbb{D})$ such that

$$
\left\|u_{n}^{0}\right\|_{H^{-1}(\mathbb{D})}=1, \quad \int_{0}^{T}\left\|b(z) U_{V}(t) u_{n}^{0}\right\|_{L^{2}(\mathbb{D})}^{2} d t \rightarrow 0, \quad n \rightarrow \infty .
$$


Inequality (7.6) implies that $u_{n}^{0}$ is bounded in $L^{2}(\mathbb{D})$, so that, after having extracted a subsequence, we have $u_{0}^{n} \rightarrow u^{0}$ in $L^{2}(\mathbb{D})$ and $u_{n}^{0} \rightarrow u^{0}$ in $H^{-1}(\mathbb{D})$. We deduce from (7.12) that

$$
\left\|u^{0}\right\|_{H^{-1}(\mathbb{D})}=1, \quad U_{V}(t) u^{0}=0 \text { on }\left\{b^{2}>0\right\} \text { for all } t \in(0, T) .
$$

The weak limit $u^{0}$ belongs to the set

$$
\mathcal{N}=\left\{f \in L^{2}(\mathbb{D}), U_{V}(t) f=0 \text { on }\left\{b^{2}>0\right\} \text { for all } t \in(0, T)\right\} .
$$

Then, by linearity, $\mathcal{N}$ is a closed vector subspace of $L^{2}(\mathbb{D})$. Inequality (7.9) proves that $\mathcal{N}$ is finite dimensional and the time independence of $V$ implies that it is a subspace of $H^{2}(\mathbb{D}) \cap H_{0}^{1}(\mathbb{D})$, stable by the action of the operator $-\Delta_{D}+V$. If not reduced to $\{0\}$, the space $\mathcal{N}$ hence contains an eigenfunction of $-\Delta_{D}+V$, vanishing on $\left\{b^{2}>0\right\}$. A classical uniqueness result for elliptic operators then implies that this does not occur. This yields $\mathcal{N}=\{0\}$ and thus $u^{0}=0$, which contradicts $\left\|u^{0}\right\|_{H^{-1}(\mathbb{D})}=1$.

7.3. Boundary observability inequality: proof of Theorem 1.3. We proceed as in the previous section: in a first step, we prove the following weakened observability inequality:

Lemma 7.5. For all $T>0$, there exists $C>0$ such that for all $u^{0} \in H_{0}^{1}(\mathbb{D})$, we have

$$
\left\|u^{0}\right\|_{H^{1}(\mathbb{D})}^{2} \leq C \int_{0}^{T}\left\|\partial_{n}\left(U_{V}(t) u^{0}\right)\right\|_{L^{2}(\Gamma)}^{2} d t+C\left\|u^{0}\right\|_{L^{2}(\mathbb{D})}^{2},
$$

With this lemma, we now conclude the proof of the observability inequality (1.6). We proceed by contradiction and suppose that the inequality

$$
\left\|u^{0}\right\|_{L^{2}(\mathbb{D})} \leq \int_{0}^{T}\left\|\partial_{n}\left(U_{V}(t) u^{0}\right)\right\|_{L^{2}(\Gamma)}^{2} d t
$$

is not satisfied. Then, there exists a sequence $\left(u_{n}^{0}\right)_{n \in \mathbb{N}}$ in $L^{2}(\mathbb{D})$ such that

$$
\left\|u_{n}^{0}\right\|_{L^{2}(\mathbb{D})}=1, \quad \int_{0}^{T}\left\|\partial_{n}\left(U_{V}(t) u_{n}^{0}\right)\right\|_{L^{2}(\Gamma)}^{2} d t \rightarrow 0, \quad n \rightarrow \infty .
$$

Then, (7.13) implies that $u_{n}^{0}$ is bounded in $H^{1}(\mathbb{D})$, so that, after having extracted a subsequence, we have $u_{n}^{0} \rightarrow u^{0}$ in $H_{0}^{1}(\mathbb{D})$ and $u_{n}^{0} \rightarrow u^{0}$ in $L^{2}(\mathbb{D})$. We deduce from (7.15) that

$$
\left\|u^{0}\right\|_{L^{2}(\mathbb{D})}=1, \quad \partial_{n}\left(U_{V}(t) u^{0}\right)=0 \text { on } \Gamma \text { for all } t \in(0, T) .
$$

From here, we discuss the two cases with different uniqueness arguments. In the case $V(t, z)=$ $V(z)$, the proof of $u=0$ follows exactly Section 7.2.2 (using unique continuation from the boundary for elliptic operators). The same conclusion holds if we assume $\left(\mathrm{UCP}_{V, \Gamma, T}\right)$. This contradicts $\left\|u^{0}\right\|_{L^{2}(\mathbb{D})}=1$, and proves (7.14). Then, (7.14) and (7.13) imply the sought observability inequality (1.6).

Proof of Lemma 7.5. Assume that (7.13) is not satisfied. Then, there exists a sequence $u_{n}^{0}$ such that

$$
\begin{aligned}
& \left\|u_{n}^{0}\right\|_{H^{1}(\mathbb{D})}^{2}=1, \\
& \left\|u_{n}^{0}\right\|_{L^{2}(\mathbb{D})}^{2} \rightarrow 0, \\
& \int_{0}^{T}\left\|\partial_{n}\left(u_{n}(t)\right)\right\|_{L^{2}(\Gamma)}^{2} d t \rightarrow 0,
\end{aligned}
$$

where, as usual, $u_{n}(t)=U_{V}(t) u_{n}^{0}$. Let us now fix $\chi_{T} \in C^{\infty}(\mathbb{R})$ such that $\chi_{T}=1$ in a neighbourhood of $[0, T], \psi \in C^{\infty}(\mathbb{R})$, such that $\psi=0$ on $(-\infty, 1]$ and $\psi=1$ on $[2,+\infty)$ and set

$$
w_{n}=B\left(D_{t}\right) \chi_{T}(t) u_{n}, \quad B\left(D_{t}\right)=\mathrm{Op}_{1}(\psi(H) \sqrt{2 H}) .
$$


Lemma 7.6. We set $A\left(D_{t}\right)=\mathrm{Op}_{1}\left(\frac{\psi(H)}{\sqrt{2 H}}\right)$. For any $R>0$ and $\varepsilon>0$, we have

$$
\begin{aligned}
& \left\|\left(D_{t}+\frac{1}{2} \Delta-V\right) w_{n}\right\|_{L^{2}((0, T) \times \mathbb{D})} \rightarrow 0 \\
& \left\|A\left(D_{t}\right) w_{n}\right\|_{L^{2}((-R, R) \times \mathbb{D})} \rightarrow 0, \\
& T / 2+o_{\varepsilon}(1) \leq\left\|w_{n}\right\|_{L^{2}((-\varepsilon, T+\varepsilon) \times \mathbb{D})}^{2} \\
& \left\|w_{n}\right\|_{L^{2}((-R, R) \times \mathbb{D})}^{2} \leq R+\varepsilon+o_{R, \varepsilon}(1), \\
& \left\|\partial_{n}\left(A\left(D_{t}\right) w_{n}\right)\right\|_{L^{2}((-R, R) \times \partial \mathbb{D})} \leq C, \\
& \left\|\partial_{n}\left(A\left(D_{t}\right) w_{n}\right)\right\|_{L^{2}((\varepsilon, T-\varepsilon) \times \Gamma)} \rightarrow 0 .
\end{aligned}
$$

Now, as $\left(w_{n}\right)$ forms a bounded sequence of $L_{\text {loc }}^{2}(\mathbb{R} \times \mathbb{D})$, we associate to a subsequence a microlocal measure $\mu_{m l}=\mu^{\infty}+\mu_{c}$ as in Section 2.5. According to (7.19) and Remark $2.5 \mu_{m l}$ satisfies the conclusions of Theorem 2.6. The measure $\mu_{c}$ vanishes as a consequence of $(7.20)$. According to $(7.23)$, the sequence $\partial_{n}\left(A\left(D_{t}\right) w_{n}\right)$ is bounded in $L^{2}((0, T) \times \mathbb{D})$, so we may again extract another subsequence and associate a microlocal measure $\mu_{m l}^{\partial}$ as in Section 2.8.3. According to $(7.24), \mu_{m l}^{\partial}=0$ on $T^{*}((\varepsilon, T-\varepsilon) \times \Gamma)$. As a consequence of Lemma $(7.2), \mu^{\infty}$ vanishes identically on $\mathbb{R}_{t} \times \mathbb{R}_{z}^{2} \times \mathbb{S}_{H, \xi}^{2}$. Thus, $w_{n}$ converges to zero in $L_{\text {loc }}^{2}(\mathbb{R} \times \mathbb{D})$, which is contradiction with (7.21). This concludes the proof of Lemma 7.5.

Proof of Lemma 7.6. Take $\tilde{\chi} \in C_{c}^{\infty}(\mathbb{R})$ such that $\tilde{\chi}=1$ on $(0, T)$ and $\chi_{T}=1$ on $\operatorname{supp}(\tilde{\chi})$. Using that $\left(D_{t}+\frac{1}{2} \Delta-V\right) u_{n}=0$, we have

$$
\begin{aligned}
\left\|\left(D_{t}+\frac{1}{2} \Delta-V\right) w_{n}\right\|_{L^{2}(0, T \times \mathbb{D})} & \leq\left\|\tilde{\chi}\left(D_{t}+\frac{1}{2} \Delta-V\right) w_{n}\right\|_{L^{2}(\mathbb{R} \times \mathbb{D})} \\
& \leq\left\|\tilde{\chi} B\left(D_{t}\right) \chi_{T}^{\prime} u_{n}\right\|_{L^{2}(\mathbb{R} \times \mathbb{D})}+\left\|\tilde{\chi}\left[V, B\left(D_{t}\right)\right] \chi_{T} u_{n}\right\|_{L^{2}(\mathbb{R} \times \mathbb{D})} \\
& \leq C\left\|\chi_{T}^{\prime} u_{n}\right\|_{L^{2}(\mathbb{R} \times \mathbb{D})}+C\left\|\chi_{T} u_{n}\right\|_{L^{2}(\mathbb{R} \times \mathbb{D})} \leq C\left\|u_{n}^{0}\right\|_{L^{2}(\mathbb{D})} \rightarrow 0,
\end{aligned}
$$

as $\tilde{\chi}=0$ on $\operatorname{supp}\left(\chi_{T}^{\prime}\right)$ and $\left[V, B\left(D_{t}\right)\right]$ is bounded on $L^{2}(\mathbb{R} \times \mathbb{D})$. This proves (7.19).

Let us now take $\tilde{\chi} \in C_{c}^{\infty}(\mathbb{R})$ such that $\tilde{\chi}=1$ on $(-R, R)$. We have

$$
\begin{aligned}
\left\|A\left(D_{t}\right) w_{n}\right\|_{L^{2}((-R, R) \times \mathbb{D})} & \leq\left\|\tilde{\chi} \mathrm{Op}_{1}\left(\psi^{2}(H)\right) \chi_{T} u_{n}\right\|_{L^{2}(\mathbb{R} \times \mathbb{D})} \\
& \leq\left\|\chi_{T} u_{n}\right\|_{L^{2}(\mathbb{R} \times \mathbb{D})} \rightarrow 0,
\end{aligned}
$$

which proves (7.20).

Let us fix now $\check{\chi} \in C_{c}^{\infty}(-\varepsilon, T+\varepsilon)$ such that $\check{\chi}=1$ in a neighbourhood of $[0, T]$, and compute

$$
\begin{aligned}
\left\|w_{n}\right\|_{L^{2}((-\varepsilon, T+\varepsilon) \times \mathbb{D})}^{2} \geq & \left\|\check{\chi} w_{n}\right\|_{L^{2}(\mathbb{R} \times \mathbb{D})}^{2}=\left\langle B\left(D_{t}\right) \check{\chi}^{2} B\left(D_{t}\right) \chi_{T} u_{n}, \chi_{T} u_{n}\right\rangle_{L^{2}(\mathbb{R} \times \mathbb{D})} \\
\geq & \left\langle\check{\chi}^{2} \mathrm{Op}_{1}\left(\psi^{2}(H)\right) D_{t}\left(\chi_{T} u_{n}\right), \chi_{T} u_{n}\right\rangle_{L^{2}(\mathbb{R} \times \mathbb{D})} \\
& +\left\langle\left[B\left(D_{t}\right), \check{\chi}^{2}\right] B\left(D_{t}\right) \chi_{T} u_{n}, \chi_{T} u_{n}\right\rangle_{L^{2}(\mathbb{R} \times \mathbb{D})},
\end{aligned}
$$

where $\left|\left\langle\left[B\left(D_{t}\right), \check{\chi}^{2}\right] B\left(D_{t}\right) \chi_{T} u_{n}, \chi_{T} u_{n}\right\rangle_{L^{2}(\mathbb{R} \times \mathbb{D})}\right| \leq\left\|\chi_{T} u_{n}\right\|_{L^{2}(\mathbb{R} \times \mathbb{D})} \rightarrow 0$. As a consequence, we have

$$
\left\|w_{n}\right\|_{L^{2}((-\varepsilon, T+\varepsilon) \times \mathbb{D})}^{2} \geq\left\langle\check{\chi}^{2} \mathrm{Op}_{1}\left(\psi^{2}(H)\right) D_{t}\left(\chi_{T} u_{n}\right), \chi_{T} u_{n}\right\rangle_{L^{2}(\mathbb{R} \times \mathbb{D})}+o(1) .
$$

On the other hand, we have

$$
\begin{aligned}
\left\langle\check{\chi}^{2} D_{t}\left(\chi_{T} u_{n}\right), \chi_{T} u_{n}\right\rangle_{L^{2}(\mathbb{R} \times \mathbb{D})}= & \left\langle\check{\chi}^{2} \mathrm{Op}_{1}\left(\psi^{2}(H)\right) D_{t}\left(\chi_{T} u_{n}\right), \chi_{T} u_{n}\right\rangle_{L^{2}(\mathbb{R} \times \mathbb{D})} \\
& +\left\langle\check{\chi}^{2} \mathrm{Op}_{1}\left(\psi^{2}(-H)\right) D_{t}\left(\chi_{T} u_{n}\right), \chi_{T} u_{n}\right\rangle_{L^{2}(\mathbb{R} \times \mathbb{D})}+o(1),
\end{aligned}
$$

where

$$
\left\langle\tilde{\chi}^{2} \mathrm{Op}_{1}\left(\psi^{2}(-H)\right) D_{t}\left(\chi_{T} u_{n}\right), \chi_{T} u_{n}\right\rangle_{L^{2}(\mathbb{R} \times \mathbb{D})}=\left\langle\check{\chi}^{2} \mathrm{Op}_{1}\left(\psi^{2}(-H) H\right)\left(\chi_{T} u_{n}\right), \chi_{T} u_{n}\right\rangle_{L^{2}(\mathbb{R} \times \mathbb{D})}
$$

Since $\check{\chi}^{2} \psi^{2}(-H) H \leq 0$ the sharp Gårding inequality then provides

$$
\left\langle\check{\chi}^{2} \mathrm{Op}_{1}\left(\psi^{2}(-H)\right) D_{t}\left(\chi_{T} u_{n}\right), \chi_{T} u_{n}\right\rangle_{L^{2}(\mathbb{R} \times \mathbb{D})} \leq\left\|\chi_{T} u_{n}\right\|_{L^{2}(\mathbb{R} \times \mathbb{D})}^{2}=o(1)
$$


This, combined with (7.25) and (7.26) now yields

$$
\begin{aligned}
\left\|w_{n}\right\|_{L^{2}((-\varepsilon, T+\varepsilon) \times \mathbb{D})}^{2} & \geq\left\langle\check{\chi}^{2} D_{t}\left(\chi_{T} u_{n}\right), \chi_{T} u_{n}\right\rangle_{L^{2}(\mathbb{R} \times \mathbb{D})}+o(1) \\
& \geq\left\langle\check{\chi}^{2} \chi_{T} D_{t} u_{n}, \chi_{T} u_{n}\right\rangle_{L^{2}(\mathbb{R} \times \mathbb{D})}+o(1) \\
& \geq\left\langle\check{\chi}^{2} \chi_{T}\left(-\frac{1}{2} \Delta+V\right) u_{n}, \chi_{T} u_{n}\right\rangle_{L^{2}(\mathbb{R} \times \mathbb{D})}+o(1) \\
& \geq \frac{1}{2}\left\langle\check{\chi}^{2} \chi_{T} \nabla u_{n}, \chi_{T} \nabla u_{n}\right\rangle_{L^{2}(\mathbb{R} \times \mathbb{D})}+o(1) \\
& \geq \frac{T}{2}\left\|\nabla u_{n}^{0}\right\|_{L^{2}(\mathbb{D})}^{2}+o(1)=\frac{T}{2}+o(1)
\end{aligned}
$$

This concludes the proof of (7.21). The proof of (7.22) follows the same arguments.

Finally, according to (7.16) and the hidden regularity result of Proposition C.1 the sequence $\partial_{n}\left(u_{n}\right)$ is bounded in $L^{2}((-R, R) \times \mathbb{D})$. Moreover, we have

$$
\begin{aligned}
\left\|\partial_{n}\left(A\left(D_{t}\right) w_{n}\right)\right\|_{L^{2}((-R, R) \times \partial \mathbb{D})} & \leq\left\|\tilde{\chi} \mathrm{Op}_{1}\left(\psi^{2}(H)\right) \chi_{T} \partial_{n}\left(u_{n}\right)\right\|_{L^{2}(\mathbb{R} \times \partial \mathbb{D})} \\
& \leq\left\|\chi_{T} \partial_{n}\left(u_{n}\right)\right\|_{L^{2}(\mathbb{R} \times \partial \mathbb{D})} \leq C .
\end{aligned}
$$

This proves (7.23). The proof of (7.24) comes from a similar computation combined with (7.18).

\section{Appendix A. From aCtion-Angle coordinates to POLAR COORDinates}

Here we develop the (painful) calculations leading to the definitions of the operators $\mathcal{A}_{E}(P)$ and $\mathcal{A}_{H}(P)$ used as a black-box in the paper. The point is that our "action-angle" coordinates $(s, \theta, E, J)$, well adapted to integrate the dynamics of the billiard flow, are not so convenient to express the Dirichlet boundary condition $(v(z)=0$ for $|z|=1)$. Actually the best coordinates in which to write the boundary condition are the polar coordinates (which below will be written as $(x=-r \sin u, y=r \cos u))$ since the boundary is simply expressed as the set $\{r=1\}$.

Let $P(s, \theta, E, J)$ be a function expressed in the new coordinates and let $\mathscr{U}$ be the Fourier integral operator defined in (3.1). The technical calculations done below are aimed at understanding how $\mathscr{U}^{*} \mathrm{Op}_{h}(P) \mathscr{U}$ acts in polar coordinates; in particular, under which conditions on the symbol $P$ the boundary condition is preserved by $\mathscr{U}^{*} \mathrm{Op}_{h}(P) \mathscr{U}$.

For our purposes we need to understand the operator $\mathscr{U}^{*} \mathrm{Op}_{h}(P) \mathscr{U}$ modulo $O\left(h^{2}\right)$. Ideally we would like to separate it into a "tangential part" (involving only angular derivation $\frac{\partial}{\partial u}$ ) and a "radial part" involving the radial derivative $\frac{\partial}{\partial r}$ in a simple way. Below we calculate the action of the operator $\mathscr{U}^{*} \mathrm{Op}_{h}(P) \mathscr{U}$ on a plane wave $e_{\xi}(z):=e^{i \frac{\left(\xi_{x} x+\xi_{y} y\right)}{h}}$ (where we use $z=(x, y)$, $\xi=\left(\xi_{x}, \xi_{y}\right)$ and $\left.|\xi|^{2}=\xi_{x}^{2}+\xi_{y}^{2}\right)$ and apply the method of stationary phase. The length of the calculation comes from the fact that we explicitly need the term of order $h$ in the expansion.

Let $P(s, \theta, E, J)$ be a smooth function (possibly depending on $h$ ), with support away from $\{E=0\}$ and inside $\{|J|<E\}$. We assume that it satisfies $\left\|\partial_{s}^{\alpha} \partial_{\theta}^{\beta} \partial_{E}^{\gamma} \partial_{J}^{\delta} P\right\|_{\infty} \leq C_{\alpha, \beta, \gamma, \delta} h^{-\gamma-\delta}$ for all integers $\alpha, \beta, \gamma, \delta$. The function $P$ may also depend on the time variable $t$ and its dual $H$, but here we omit them from the notation since they are transparent in the calculation. Using the 
definition (3.1) and unfolding all the integrals, we write

$$
\begin{aligned}
& \mathscr{U}^{*} \mathrm{Op}_{h}(P) \mathscr{U} e_{\xi}(x, y) \\
& =(2 \pi h)^{-5} \int P\left(s, \theta, E^{\prime}, j\right) e^{\frac{i j\left(\theta-\theta^{\prime}\right)}{h}} e^{\frac{i E^{\prime}\left(s-s^{\prime}\right)}{h}} e^{-i \frac{S\left(x^{\prime}, y^{\prime}, \theta^{\prime}, s^{\prime}, E\right)}{h}} e^{i \frac{\left(\xi_{x} x^{\prime}+\xi_{y} y^{\prime}\right)}{h}} e^{i \frac{S\left(x, y, \theta, s, E^{\prime \prime}\right)}{h}} \\
& a(E) a\left(E^{\prime \prime}\right) d \theta d s d E^{\prime \prime} d x^{\prime} d y^{\prime} d E d \theta^{\prime} d s^{\prime} d E^{\prime} d j \\
& =(2 \pi h)^{-3} \int P\left(s, \theta, E^{\prime}, j\right) e^{\frac{i j\left(\theta-\theta_{0}\right)}{h}} e^{\frac{i E^{\prime}\left(s-s^{\prime}\right)}{h}} e^{i \frac{s^{\prime}|\xi|}{h}} e^{i \frac{S\left(x, y, \theta, s, E^{\prime \prime}\right)}{h}} \frac{a(|\xi|)}{|\xi|} a\left(E^{\prime \prime}\right) d \theta d s d E^{\prime \prime} d s^{\prime} d E^{\prime} d j \\
& =(2 \pi h)^{-2} \int P(s, \theta,|\xi|, j) e^{\frac{i j\left(\theta-\theta_{0}\right)}{h}} e^{i \frac{s|\xi|}{h}} e^{i \frac{S\left(x, y, \theta, s, E^{\prime \prime}\right)}{h}} \frac{a(|\xi|)}{|\xi|} a\left(E^{\prime \prime}\right) d \theta d E^{\prime \prime} d s d j \\
& =(2 \pi h)^{-1} \int\left(P(s(x, y, \theta), \theta,|\xi|, j) a(|\xi|)-i h \partial_{s} P(s(x, y, \theta), \theta,|\xi|, j) a^{\prime}(|\xi|)\right) \\
& e^{\frac{i j\left(\theta-\theta_{0}\right)}{h}} e^{i \frac{-|\xi| \sin \theta x+|\xi| \cos \theta y}{h}} \frac{a(|\xi|)}{|\xi|} d \theta d j \quad+\frac{O\left(h^{2}\right)}{\inf _{P(s, \theta, E, J) \neq 0}|E|^{2}},
\end{aligned}
$$

where $a(E)=\sqrt{E},\left(s_{0}, \theta_{0},|\xi|, j_{0}\right)=\Phi^{-1}\left(x, y, \xi_{x}, \xi_{y}\right)$ and $s(x, y, \theta)=-x \sin \theta+y \cos \theta$. By standard estimates on pseudodifferential operators, the remainder term will correspond to an estimate in the $L_{\text {comp }}^{2} \longrightarrow L_{\text {loc }}^{2}$ norm of operators.

Let $(x, y)=(-r \sin u, r \cos u)$, so that $r=\sqrt{x^{2}+y^{2}}, u=\arccos y / r$, and $s(x, y, \theta)=r \cos (\theta-$ $u)$. We are left with

$$
\begin{aligned}
(2 \pi h)^{-1} \frac{a(|\xi|)}{|\xi|} \int(P(r \cos (\theta-u), \theta,|\xi|, j) a(|\xi|) & \\
& \left.-i h \partial_{s} P(r \cos (\theta-u), \theta,|\xi|, j) a^{\prime}(|\xi|)\right) e^{\frac{i j\left(\theta-\theta_{0}\right)}{h}} e^{i \frac{|\xi| r \cos (\theta-u)}{h}} d \theta d j .
\end{aligned}
$$

Now we apply stationary phase w.r.t. $\theta$ (while $j$ is kept fixed, since our symbols can be rapidly oscillating in $j$ ). We start with the $P$ term. The $i h \partial_{s} P$-term can be treated exactly the same way. Fixing $j$ and looking at the $\theta$-integral, we let

$$
\begin{aligned}
I & =(2 \pi h)^{-1 / 2} \int_{0}^{2 \pi} P(r \cos (\theta-u), \theta,|\xi|, j) e^{\frac{i j\left(\theta-\theta_{0}\right)}{h}} e^{i \frac{|\xi| r \cos (\theta-u)}{h}} d \theta \\
& =(2 \pi h)^{-1 / 2} \int_{u-\pi / 2}^{u+\pi / 2} \cdots+(2 \pi h)^{-1 / 2} \int_{u+\pi / 2}^{u+3 \pi / 2} \cdots .
\end{aligned}
$$

The phase in $I$ has 2 critical points $\theta=u+\theta_{1}, u+\theta_{2}$, where $\theta_{i}$ are the solutions of $j-|\xi| r \sin \theta=0$. Since we are assuming that $P(s, \theta, E, j)$ is supported in $\{|j|<E\}$, these two solutions are distinct for $r$ close to 1 , and correspond to non-degenerate stationary points (in all that follows we consider that $r$ is close to 1 since this calculation only serves to understand $\mathscr{U}^{*} \mathrm{Op}_{h}(P) \mathscr{U}$ near the boundary of the disk). We will denote by $\theta_{1}(r, E, j), \theta_{2}(r, E, j)$ the solutions of $j-E r \sin \theta=0$. To fix ideas, $\theta_{1}$ will be the one with $\cos \theta_{1}>0$ and $\theta_{2}$ the one with $\cos \theta_{2}<0$ (that is, $\theta_{1} \in(-\pi / 2, \pi / 2), \theta_{2} \in$ $(\pi / 2,3 \pi / 2))$. Below, $E$ will always take the value $E=|\xi|$.

According to the method of stationary phase, the asymptotic expansion of the integral $I$ may be obtained, modulo $O\left(h^{2}\right)$, by replacing $P$ by its Taylor expansion at order 2 at each critical point:

$$
\begin{aligned}
P(r \cos (\theta-u), \theta) \sim P\left(r \cos \theta_{i}, u+\theta_{i}\right)+\left(\theta-u-\theta_{i}\right) \frac{d}{d \theta} P\left(r \cos \theta_{i}, u+\theta_{i}\right) & \\
& +\left(\theta-u-\theta_{i}\right)^{2} \frac{1}{2} \frac{d^{2}}{d \theta^{2}} P\left(r \cos \theta_{i}, u+\theta_{i}\right)
\end{aligned}
$$

(we momentarily drop the $j$ and $E$ variables from the notation since they are fixed in the upcoming calculation).

Remark A.1. Denoting by $\partial_{1}=\partial_{s}, \partial_{2}=\partial_{\theta}$ (to avoid possible confusion), we have

$$
\frac{d}{d \theta} P(r \cos \theta, u+\theta)=\partial_{2} P(r \cos \theta, u+\theta)-r \sin \theta \partial_{1} P(r \cos \theta, u+\theta),
$$


and

$$
\begin{aligned}
\frac{d^{2}}{d \theta^{2}} P(r \cos \theta, u+\theta)=\partial_{2}^{2} P(r \cos \theta & , u+\theta)-r \cos \theta \partial_{1} P(r \cos \theta, u+\theta) \\
& -r \sin \theta \partial_{2} \partial_{1} P(r \cos \theta, u+\theta)+r^{2} \sin ^{2} \theta \partial_{1}^{2} P(r \cos \theta, u+\theta) .
\end{aligned}
$$

To use integration by parts, it is convenient to rewrite (A.1) using $j-\operatorname{Er} \sin (\theta-u)$ (the derivative of the phase) instead of $\left(\theta-u-\theta_{i}\right)$ (where $\theta_{i}$ stands short for $\left.\theta_{i}(r, E, j)\right)$. Starting with

$$
j-\operatorname{Er} \sin (\theta-u) \sim \operatorname{Er}\left[-\cos \theta_{i}\left(\theta-u-\theta_{i}\right)+\frac{\sin \theta_{i}}{2}\left(\theta-u-\theta_{i}\right)^{2}\right],
$$

equation (A.1) can be rewritten as

$$
\begin{aligned}
& P(r \cos (\theta-u), \theta) \sim P\left(r \cos \theta_{i}, u+\theta_{i}\right)-\frac{(j-E r \sin (\theta-u))}{E r \cos \theta_{i}} \frac{d}{d \theta} P\left(r \cos \theta_{i}, u+\theta_{i}\right) \\
& \quad+\frac{(j-E r \sin (\theta-u))^{2}}{\left(E r \cos \theta_{i}\right)^{2}}\left[\frac{\sin \theta_{i}}{2 \cos \theta_{i}} \frac{d}{d \theta} P\left(r \cos \theta_{i}, u+\theta_{i}\right)+\frac{1}{2} \frac{d^{2}}{d \theta^{2}} P\left(r \cos \theta_{i}, u+\theta_{i}\right)\right] .
\end{aligned}
$$

Remark A.2. Important remark about symmetry. We keep denoting $\theta_{i}$ for $\theta_{i}(r, E, j)$. We first note that $\theta_{2}=\pi-\theta_{1}, \cos \theta_{1}=-\cos \theta_{2}, \sin \theta_{1}=\sin \theta_{2}$.

Moreover, if $P$ satisfies the symmetry condition (B), we have for $r=1$ (restoring in our notation the dependence of $P$ on the full set of variables)

$$
P\left(\cos \theta_{1}, u+\theta_{1}, E, j\right)=P\left(\cos \theta_{2}, u+\theta_{2}, E, j\right) .
$$

And similarly for all partial derivatives of $P$ if we assume the stronger symmetry condition $(C)$.

Here we don't necessarily want to assume that $P$ is symmetric; but, motivated by the previous remark, we introduce the following notation:

$$
\begin{aligned}
P^{\sigma}(r, \theta, E, j) & :=\frac{P\left(r \cos \theta_{1}, \theta+\theta_{1}, E, j\right)+P\left(-r \cos \theta_{1}, \theta+\pi-\theta_{1}, E, j\right)}{2} \\
& =\frac{P\left(r \cos \theta_{1}, \theta+\theta_{1}, E, j\right)+P\left(r \cos \theta_{2}, \theta+\theta_{2}, E, j\right)}{2}
\end{aligned}
$$

$$
\begin{aligned}
P^{\alpha}(r, \theta, E, j) & :=\frac{P\left(r \cos \theta_{1}, \theta+\theta_{1}, E, j\right)-P\left(-r \cos \theta_{1}, \theta+\pi-\theta_{1}, E, j\right)}{2} \\
& =\frac{P\left(r \cos \theta_{1}, \theta+\theta_{1}, E, j\right)-P\left(r \cos \theta_{2}, \theta+\theta_{2}, E, j\right)}{2}
\end{aligned}
$$

for $\theta_{1}=\theta_{1}(r, E, j), \theta_{2}=\theta_{2}(r, E, j)$ defined previously, so that

$$
\begin{aligned}
& P\left(r \cos \theta_{1}, \theta+\theta_{1}, E, j\right)=P^{\sigma}(r, \theta, E, j)+P^{\alpha}(r, \theta, E, j), \\
& P\left(r \cos \theta_{2}, \theta+\theta_{2}, E, j\right)=P^{\sigma}(r, \theta, E, j)-P^{\alpha}(r, \theta, E, j) .
\end{aligned}
$$

Modulo $O(h)$ the asymptotic expansion of the integral $I$ looks as follows: 
(A.6)

$$
\begin{aligned}
& \text { (A.6) } \begin{array}{r}
(2 \pi h)^{-1 / 2} \int_{u-\pi / 2}^{u+\pi / 2} P\left(r \cos \theta_{1}, u+\theta_{1}, E, j\right) e^{\frac{i j\left(\theta-\theta_{0}\right)}{h}} e^{i \frac{E r \cos (\theta-u)}{h}} d \theta \\
+(2 \pi h)^{-1 / 2} \int_{u+\pi / 2}^{u+3 \pi / 2} P\left(r \cos \theta_{2}, u+\theta_{2}, E, j\right) e^{\frac{i j\left(\theta-\theta_{0}\right)}{h}} e^{i \frac{E r \cos (\theta-u)}{h}} d \theta \\
=(2 \pi h)^{-1 / 2} \int_{0}^{2 \pi} P^{\sigma}(r, u, E, j) e^{\frac{i j\left(\theta-\theta_{0}\right)}{h}} e^{i \frac{E r \cos (\theta-u)}{h}} d \theta \\
+(2 \pi h)^{-1 / 2} \int_{0}^{2 \pi} \frac{P^{\alpha}(r, u, E, j)}{E \cos \theta_{1}(r, E, j)}\left(E \cos (\theta-u)-i h \frac{1}{2 r \cos ^{2} \theta_{1}(r, E, j)}\right) e^{\frac{i j\left(\theta-\theta_{0}\right)}{h}} e^{i \frac{E r \cos (\theta-u)}{h}} d \theta \\
=(2 \pi h)^{-1 / 2} \int_{0}^{2 \pi} P^{\sigma}(r, u, E, j) e^{\frac{i j(u-\theta)}{h}} e^{i \frac{E r \cos \left(\theta-\theta_{0}\right)}{h}} d \theta \\
+(2 \pi h)^{-1 / 2} \int_{0}^{2 \pi} \frac{P^{\alpha}(r, u, E, j)}{E \cos \theta_{1}(r, E, j)}\left(E \cos \left(\theta-\theta_{0}\right)-i h \frac{1}{2 r \cos ^{2} \theta_{1}(r, E, j)}\right) e^{\frac{i j(u-\theta)}{h}} e^{i \frac{E r \cos \left(\theta-\theta_{0}\right)}{h}} d \theta
\end{array}
\end{aligned}
$$

We note that $e^{i \frac{E r \cos \left(\theta-\theta_{0}\right)}{h}}=e^{i \frac{\left(\xi_{x} x^{\prime}+\xi_{y} y^{\prime}\right)}{h}}$ if $\left(x^{\prime}, y^{\prime}\right)=(-r \sin \theta, r \cos \theta)$, and $E \cos \left(\theta-\theta_{0}\right) e^{i \frac{E r \cos \left(\theta-\theta_{0}\right)}{h}}=$ $h D_{r} e^{i\left(\xi_{x} x^{\prime}+\xi_{y} y^{\prime}\right) / h}$ where $D_{r}=\frac{1}{i} \partial_{r}$.

Other terms of order $h$. Apart from the term of order $h$ arising in the last line of (A.6), other terms of order $h$ come from evaluation of the integrals

$$
\begin{aligned}
& (2 \pi h)^{-1 / 2} \int_{u-\pi / 2}^{u+\pi / 2}\left[\frac{-(j-E r \sin (\theta-u))}{E r \cos \theta_{i}} \frac{d}{d \theta} P\left(u+\theta_{1}, r \cos \theta_{1}\right)\right. \\
& \left.+\frac{(j-E r \sin (\theta-u))^{2}}{\left(E r \cos \theta_{1}\right)^{2}}\left(\frac{\sin \theta_{1}}{2 \cos \theta_{1}} \frac{d}{d \theta} P\left(u+\theta_{1}, r \cos \theta_{1}\right)+\frac{1}{2} \frac{d^{2}}{d \theta^{2}} P\left(u+\theta_{1}, r \cos \theta_{1}\right)\right)\right] \\
& e^{\frac{i j\left(\theta-\theta_{0}\right)}{h}} e^{i \frac{E r \cos (\theta-u)}{h}} d \theta \\
& +(2 \pi h)^{-1 / 2} \int_{u+\pi / 2}^{u+3 \pi / 2}\left[\frac{-(j-E r \sin (\theta-u))}{E r \cos \theta_{2}} \frac{d}{d \theta} P\left(u+\theta_{2}, r \cos \theta_{2}\right)\right. \\
& \left.+\frac{(j-E r \sin (\theta-u))^{2}}{\left(E r \cos \theta_{2}\right)^{2}}\left(\frac{\sin \theta_{2}}{2 \cos \theta_{2}} \frac{d}{d \theta} P\left(u+\theta_{2}, r \cos \theta_{2}\right)+\frac{1}{2} \frac{d^{2}}{d \theta^{2}} P\left(u+\theta_{2}, r \cos \theta_{2}\right)\right)\right] \\
& e^{\frac{i j\left(\theta-\theta_{0}\right)}{h}} e^{i \frac{E r \cos (\theta-u)}{h}} d \theta
\end{aligned}
$$


After integrations by parts w.r.t. $\theta$ (using the fact that $j-\operatorname{Er} \sin (\theta-u$ ) is the derivative of the phase $\left.j\left(\theta-\theta_{0}\right)+\operatorname{Er} \cos (\theta-u)\right)$, this becomes

$$
\begin{aligned}
& (2 \pi h)^{-1 / 2} i h \int_{u-\pi / 2}^{u+\pi / 2} \frac{1}{\left(E r \cos \theta_{1}\right)^{2}} \operatorname{Er} \cos (\theta-u) \\
& {\left[\frac{\sin \theta_{1}}{2 \cos \theta_{1}} \frac{d}{d \theta} P\left(u+\theta_{1}, r \cos \theta_{1}\right)+\frac{1}{2} \frac{d^{2}}{d \theta^{2}} P\left(u+\theta_{1}, r \cos \theta_{1}\right)\right] e^{\frac{i j\left(\theta-\theta_{0}\right)}{h} e^{i \frac{E r \cos (\theta-u)}{h}} d \theta}} \\
& +(2 \pi h)^{-1 / 2} i h \int_{u+\pi / 2}^{u+3 \pi / 2} \frac{1}{\left(E r \cos \theta_{2}\right)^{2}} \operatorname{Er} \cos (\theta-u) \\
& {\left[\frac{\sin \theta_{2}}{2 \cos \theta_{2}} \frac{d}{d \theta} P\left(u+\theta_{2}, r \cos \theta_{2}\right)+\frac{1}{2} \frac{d^{2}}{d \theta^{2}} P\left(u+\theta_{2}, r \cos \theta_{2}\right)\right] e^{\frac{i j\left(\theta-\theta_{0}\right)}{h}} e^{i \frac{E r \cos (\theta-u)}{h}} d \theta} \\
& =(2 \pi h)^{-1 / 2} i h \int_{u-\pi / 2}^{u+\pi / 2} \frac{1}{\left(E r \cos \theta_{1}\right)} \\
& {\left[\frac{\sin \theta_{1}}{2 \cos \theta_{1}} \frac{d}{d \theta} P\left(u+\theta_{1}, r \cos \theta_{1}\right)+\frac{1}{2} \frac{d^{2}}{d \theta^{2}} P\left(u+\theta_{1}, r \cos \theta_{1}\right)\right] e^{\frac{i j\left(\theta-\theta_{0}\right)}{h}} e^{i \frac{E r \cos (\theta-u)}{h}} d \theta} \\
& +(2 \pi h)^{-1 / 2} i h \int_{u+\pi / 2}^{u+3 \pi / 2} \frac{1}{\left(E r \cos \theta_{2}\right)} \\
& {\left[\frac{\sin \theta_{2}}{2 \cos \theta_{2}} \frac{d}{d \theta} P\left(u+\theta_{2}, r \cos \theta_{2}\right)+\frac{1}{2} \frac{d^{2}}{d \theta^{2}} P\left(u+\theta_{2}, r \cos \theta_{2}\right)\right] e^{\frac{i j\left(\theta-\theta_{0}\right)}{h}} e^{i \frac{E r \cos (\theta-u)}{h}} d \theta+O\left(h^{2}\right)}
\end{aligned}
$$

Here $\frac{d}{d \theta} P\left(u+\theta_{i}, r \cos \theta_{i}\right)$ and $\frac{d^{2}}{d \theta^{2}} P\left(u+\theta_{i}, r \cos \theta_{i}\right)$ may be replaced by their expressions in terms of partial derivatives of $P$, as in Remark A.1.

We summarize our calculations in the following proposition.

Proposition A.3. Modulo a term of order $\frac{O\left(h^{2}\right)}{\inf _{P(s, \theta, E, J) \neq 0}|E|^{2}}$ in the $L_{\mathrm{comp}}^{2} \longrightarrow L_{\mathrm{loc}}^{2}$-norm of operators, $\mathscr{U}^{*} \mathrm{Op}_{h}(P) \mathscr{U}$ acts as follows. For $\xi=\left(\xi_{x}, \xi_{y}\right), E=|\xi|$ and $(x, y)=(-r \sin u, r \cos u)$, we have

$$
\begin{aligned}
\mathscr{U}^{*} \mathrm{Op}_{h}(P) \mathscr{U} e_{\xi}(x, y) & =\frac{1}{2 \pi h} \int A(r, u, E, j) e^{\frac{i j(u-\theta)}{h}} e_{\xi}(-r \sin \theta, r \cos \theta) d \theta d j \\
+ & \frac{1}{2 \pi h} \int B(r, u, E, j) e^{\frac{i j(u-\theta)}{h}} h D_{r} e_{\xi}(-r \sin \theta, r \cos \theta) d \theta d j \\
& +\frac{i h}{2 \pi h} \int C(r, u, E, j) e^{\frac{i j(u-\theta)}{h}} e_{\xi}(-r \sin \theta, r \cos \theta) d \theta d j \\
& +\frac{i h}{2 \pi h} \int D(r, u, E, j) e^{\frac{i j(u-\theta)}{h}} h D_{r} e_{\xi}(-r \sin \theta, r \cos \theta) d \theta d j
\end{aligned}
$$

where

$$
\begin{gathered}
A(r, u, E, j)=P^{\sigma}(r, u, E, j) \\
B(r, u, E, j)=\frac{P^{\alpha}(r, u, E, j)}{E \cos \theta_{1}(r, E, j)} \\
C(r, u, E, j)=-\frac{1}{2 E} \partial_{s} P^{\sigma}(r, u, E, j)+c^{\sigma}(r, u, E, j)-\frac{1}{2 r \cos ^{2} \theta_{1}(r, E, j)} \frac{P^{\alpha}(r, u, E, j)}{E \cos \theta_{1}(r, E, j)} \\
D(r, u, E, j)=-\frac{1}{2 E} \frac{\partial_{s} P^{\alpha}(r, u, E, j)}{E \cos \theta_{1}(r, E, j)}+\frac{c^{\alpha}(r, u, E, j)}{E \cos \theta_{1}(r, E, j)}
\end{gathered}
$$


with the notation $P^{\sigma}, P^{\alpha}$ of (A.4), (A.5), and where, in addition

$$
\begin{aligned}
& c(s, \theta, E, j)=\frac{1}{(E s)}\left[\frac{j}{2 E s}\left(\partial_{2} P(s, \theta, E, j)-\frac{j}{E} \partial_{1} P(s, \theta, E, j)\right)\right. \\
& \left.\quad+\frac{1}{2}\left(\partial_{2}^{2} P(s, \theta, E, j)-s \partial_{1} P(s, \theta, E, j)-\frac{j}{E} \partial_{2} \partial_{1} P(s, \theta, E, j)+\frac{j^{2}}{E^{2}} \partial_{1}^{2} P(s, \theta, E, j)\right)\right]
\end{aligned}
$$

is calculated so that $c\left(u+\theta_{i}, r \cos \theta_{i}, E, j\right)$ equals

$$
\frac{1}{\left(E r \cos \theta_{i}\right)}\left[\frac{\sin \theta_{i}}{2 \cos \theta_{i}} \frac{d}{d \theta} P\left(u+\theta_{i}, r \cos \theta_{i}\right)+\frac{1}{2} \frac{d^{2}}{d \theta^{2}} P\left(u+\theta_{i}, r \cos \theta_{i}\right)\right]
$$

(the expression appearing in the last line of (A.7)).

Note that $A, B, C, D$ are real-valued functions if $P$ is.

\section{Appendix B. Commutators}

In the following formal calculations, it will be convenient to introduce the following notation.

$\mathcal{A}_{E}(P)$ is the operator whose action on $e_{\xi}$ at $(-r \sin u, r \cos u)$ is defined by

$$
\begin{aligned}
\frac{1}{2 \pi h} \int A(r, u, E, j) e^{\frac{i j(u-\theta)}{h}} e_{\xi}(-r \sin \theta, r \cos \theta) d \theta d j \\
+\frac{1}{2 \pi h} \int B(r, u, E, j) e^{\frac{i j(u-\theta)}{h}} h D_{r} e_{\xi}(-r \sin \theta, r \cos \theta) d \theta d j \\
+\frac{i h}{2 \pi h} \int C(r, u, E, j) e^{\frac{i j(u-\theta)}{h}} e_{\xi}(-r \sin \theta, r \cos \theta) d \theta d j \\
+\frac{i h}{2 \pi h} \int D(r, u, E, j) e^{\frac{i j(u-\theta)}{h}} h D_{r} e_{\xi}(-r \sin \theta, r \cos \theta) d \theta d j \\
=: I_{E}(P)+I I_{E}(P)+i h I I I_{E}(P)+i h I V_{E}(P)
\end{aligned}
$$

where $E=|\xi|$. We have shown that $\mathcal{A}_{E}(P)$ coincides with $\mathscr{U}^{*} \mathrm{Op}(P) \mathscr{U}$ modulo $\frac{O\left(h^{2}\right)}{\inf _{P(s, \theta, E, J) \neq 0}|E|^{2}}$ in the $L_{\text {comp }}^{2} \longrightarrow L_{\text {loc }}^{2}$-norm of operators.

We now define $\mathcal{A}_{H}(P)$ as the operator whose action on $e_{\xi}$ at $(-r \sin u, r \cos u)$ is defined by

$$
\begin{aligned}
\frac{1}{2 \pi h} \int A(r, u, \sqrt{2 H}, j) e^{\frac{i j(u-\theta)}{h}} e_{\xi}(-r \sin \theta, r \cos \theta) d \theta d j \\
+\frac{1}{2 \pi h} \int B(r, u, \sqrt{2 H}, j) e^{\frac{i j(u-\theta)}{h}} h D_{r} e_{\xi}(-r \sin \theta, r \cos \theta) d \theta d j \\
+\frac{i h}{2 \pi h} \int C(r, u, \sqrt{2 H}, j) e^{\frac{i j(u-\theta)}{h}} e_{\xi}(-r \sin \theta, r \cos \theta) d \theta d j \\
+\frac{i h}{2 \pi h} \int D(r, u, \sqrt{2 H}, j) e^{\frac{i j(u-\theta)}{h}} h D_{r} e_{\xi}(-r \sin \theta, r \cos \theta) d \theta d j \\
=: I_{H}(P)+I I_{H}(P)+i h I I I_{H}(P)+i h I V_{H}(P)
\end{aligned}
$$

In other words, in the definition of $\mathcal{A}_{E}(P)$ we have replaced $E$ with $\sqrt{2 H}$ in the symbols. For us, $\mathcal{A}_{H}(P)$ is a very convenient operator since we have

$$
I_{H}(P)=A\left(r, u, \sqrt{2 h D_{t}}, h D_{u}\right), \quad I I I_{H}(P)=C\left(r, u, \sqrt{2 h D_{t}}, h D_{u}\right)
$$

(so that they do not involve any derivative w.r.t. $r$ ) and

$$
I I_{H}(P)=B\left(r, u, \sqrt{2 h D_{t}}, h D_{u}\right) \circ h D_{r}, \quad I V_{H}(P)=D\left(r, u, \sqrt{2 h D_{t}}, h D_{u}\right) \circ h D_{r}
$$

which is is only of degree 1 w.r.t. $r$.

We would like to use everywhere $\mathcal{A}_{H}(P)$ instead of $\mathcal{A}_{E}(P)$. This is possible thanks to the following lemma: 
Lemma B.1. If $u_{h}$ is a solution to the Schrödinger equation (1.1) satisfying in addition the assumptions of Remark 2.3, then we have

$$
\begin{aligned}
\left\langle u_{h}, \mathcal{A}_{E}(P) u_{h}\right\rangle_{L^{2}\left(\mathbb{R}^{2} \times \mathbb{R}^{2} \times \mathbb{R}\right)}-\left\langle u_{h}, \mathcal{A}_{H}(P) u_{h}\right\rangle_{L^{2}\left(\mathbb{R}^{2} \times \mathbb{R}^{2} \times \mathbb{R}\right)} & \\
& =O\left(h\left\|\partial_{E} P\right\|_{\infty}\left(h \inf _{P(s, \theta, E, J) \neq 0}|E|\right)^{-1 / 2-\epsilon}\right) .
\end{aligned}
$$

For instance if $P$ has bounded derivatives and $\inf _{P(s, \theta, E, J) \neq 0}|E|$ is bounded away from 0 independently of $h$, the error above is $O\left(h^{1 / 2-\epsilon}\right)$.

The goal of this section is to calculate explicitly (in terms of $P$ ) the expression of the commutator $\left[\Delta, \mathcal{A}_{H}(P)\right]$, where $\Delta$ is the laplacian on $\mathbb{R}^{2}$. This could, in principle, be done by brutal calculation, using the expression of the laplacian in polar coordinates $\left(\Delta_{r, u}=\frac{\partial^{2}}{\partial_{r}^{2}}+\frac{1}{r} \frac{\partial}{\partial r}+\frac{1}{r^{2}} \frac{\partial^{2}}{\partial_{u}^{2}}\right)$. But this is too cumbersome and we try a less frontal approach. We want to use the fact that $\left[\Delta, \mathcal{A}_{E}(P)\right]$ is known (from the exact Egorov theorem, equation (B.1) below) and to see how the calculus is modified when we replace $\mathcal{A}_{E}(P)$ by $\mathcal{A}_{H}(P)$.

Recall from Lemma 3.1 and formula (2.10) that we have the exact formula (without remainder term)

$$
\left[-\frac{i h \Delta}{2}, \mathscr{U}^{*} \mathrm{Op}_{h}(P) \mathscr{U}\right]=\mathscr{U}^{*} \mathrm{Op}_{h}\left(E \partial_{1} b-\frac{i h}{2} \partial_{1}^{2} b\right) \mathscr{U}
$$

B.1. Formal calculation of $\left[\Delta, \mathcal{A}_{E}(P)\right]$. We use the expression of $\nabla$ in polar coordinates: $\nabla=$ $\left(\partial_{r}, r^{-1} \partial_{u}\right)$ in the orthonormal frame $\left(e_{r}, e_{u}\right)$. We also use the formula $\Delta(f g)=f \Delta g+2 \nabla f \cdot \nabla g+$ $g \Delta f$. We obtain the following expression of $\left[\Delta, I_{E}(P)\right]$ applied to $e_{\xi}$ at $(x, y)=(-r \sin u, r \cos u)$ :

$$
\begin{aligned}
&(2 \pi h)^{-1} \int_{u-\pi / 2}^{u+\pi / 2} \Delta_{r, u} A(r, u, E, j) e^{\frac{i j\left(\theta-\theta_{0}\right)}{h}} e^{i \frac{E r \cos (\theta-u)}{h}} d \theta d j \\
&+\frac{2 i}{h}(2 \pi h)^{-1} \int_{u-\pi / 2}^{u+\pi / 2} \partial_{r} A(r, u, E, j) E \cos (\theta-u) e^{\frac{i j\left(\theta-\theta_{0}\right)}{h}} e^{i \frac{E r \cos (\theta-u)}{h}} d \theta d j \\
&+\frac{2 i}{h}(2 \pi h)^{-1} \int_{u-\pi / 2}^{u+\pi / 2} r^{-2} \partial_{u} A(r, u, E, j) j e^{\frac{i j\left(\theta-\theta_{0}\right)}{h}} e^{i \frac{E r \cos (\theta-u)}{h}} d \theta d j
\end{aligned}
$$

Note that the details of the calculations are actually not important, we only need to know "what the calculations look like" at a formal level (in particular, small errors of calculation are harmless).

Similarly, $\left[\Delta, I I_{E}(P)\right]$ has the expression

$$
\begin{aligned}
& (2 \pi h)^{-1} \int_{u-\pi / 2}^{u+\pi / 2} \Delta_{r, u} B(r, u, E, j) E \cos (\theta-u) e^{\frac{i j\left(\theta-\theta_{0}\right)}{h}} e^{i \frac{E r \cos (\theta-u)}{h}} d \theta d j \\
& +\frac{2 i}{h}(2 \pi h)^{-1} \int_{u-\pi / 2}^{u+\pi / 2} \partial_{r} B(r, u, E, j)(E \cos (\theta-u))^{2} e^{\frac{i j\left(\theta-\theta_{0}\right)}{h}} e^{i \frac{E r \cos (\theta-u)}{h}} d \theta d j \\
& +\frac{2 i}{h}(2 \pi h)^{-1} \int_{u-\pi / 2}^{u+\pi / 2} r^{-2} \partial_{u} B(r, u, E, j) j E \cos (\theta-u) e^{\frac{i j\left(\theta-\theta_{0}\right)}{h}} e^{i \frac{E r \cos (\theta-u)}{h}} d \theta d j \\
& \quad=(2 \pi h)^{-1} \int_{u-\pi / 2}^{u+\pi / 2} \Delta_{r, u} B(r, u, E, j) E \cos (\theta-u) e^{\frac{i j\left(\theta-\theta_{0}\right)}{h}} e^{i \frac{E r \cos (\theta-u)}{h}} d \theta d j \\
& +\frac{2 i}{h}(2 \pi h)^{-1} \int_{u-\pi / 2}^{u+\pi / 2} \partial_{r} B(r, u, E, j)\left[\left(E \cos \left(\theta_{1}\right)\right)^{2}+i h \frac{\cos \theta_{1}}{(E r)^{2}} e^{\frac{i j\left(\theta-\theta_{0}\right)}{h}} e^{i \frac{E r \cos (\theta-u)}{h}} d \theta d j\right. \\
& +\frac{2 i}{h}(2 \pi h)^{-1} \int_{u-\pi / 2}^{u+\pi / 2} r^{-2} \partial_{u} B(r, u, E, j) j E \cos (\theta-u) e^{\frac{i j\left(\theta-\theta_{0}\right)}{h}} e^{i \frac{E r \cos (\theta-u)}{h}} d \theta d j+O\left(h^{2}\right)
\end{aligned}
$$

Similar calculations can be done for $\left[\Delta, I I I_{E}(P)\right]$ and $\left[\Delta, I V_{E}(P)\right]$. We do not need the explicit expressions, but need only to note that it gives a final expression of $\left[-i h \Delta / 2, \mathcal{A}_{E}(P)\right]$ applied to 
$e_{\xi}$ at $(-r \sin u, r \cos u)$ in the form:

$$
\begin{aligned}
\frac{1}{2 \pi h} \int K(r, u, E, j) e^{\frac{i j(u-\theta)}{h}} e^{i \frac{E r \cos \left(\theta-\theta_{0}\right)}{h}} d \theta d j & \\
+ & \frac{1}{2 \pi h} \int L(r, u, E, j) E \cos \left(\theta-\theta_{0}\right) e^{\frac{i j(u-\theta)}{h}} e^{i \frac{E r \cos \left(\theta-\theta_{0}\right)}{h}} d \theta d j \\
& +\frac{i h}{2 \pi h} \int M(r, u, E, j) e^{\frac{i j(u-\theta)}{h}} e^{i \frac{E r \cos \left(\theta-\theta_{0}\right)}{h}} d \theta d j \\
+ & \frac{i h}{2 \pi h} \int N(r, u, E, j) E \cos \left(\theta-\theta_{0}\right) e^{\frac{i j(u-\theta)}{h}} e^{i \frac{E r \cos \left(\theta-\theta_{0}\right)}{h} d \theta d j} \\
+(2 \pi h)^{-1} & \int \partial_{r} B(r, u, E, j)\left[\left(E \cos \left(\theta_{1}\right)\right)^{2}+i h \frac{\cos \theta_{1}}{(E r)^{2}}\right] e^{\frac{i j\left(\theta-\theta_{0}\right)}{h}} e^{i \frac{E r \cos (\theta-u)}{h}} d \theta d j \\
& +(2 \pi h)^{-1} \int i h \partial_{r} D(r, u, E, j)\left(E \cos \left(\theta_{1}\right)\right)^{2} e^{\frac{i j\left(\theta-\theta_{0}\right)}{h}} e^{i \frac{E r \cos (\theta-u)}{h}} d \theta d j
\end{aligned}
$$

Note that the two last lines may obviously be incorporated into the previous terms; but we shall see later why it is convenient to keep them separate.

The functions $K, L, M, N$ are partial differential operators applied to $A, B, C, D$, and could in principle be expressed explicitly in terms of $P$, but we actually do not need these expressions.

B.2. Identification. We know from (B.1) that $\left[-\frac{i h \Delta}{2}, \mathscr{U}^{*} \mathrm{Op}_{h}(P) \mathscr{U}\right]=\mathscr{U}^{*} \mathrm{Op}_{h}\left(E \partial_{1} P-\frac{i h}{2} \partial_{1}^{2} P\right) \mathscr{U}=$ $\mathcal{A}_{E}\left(E \partial_{1} P-\frac{i h}{2} \partial_{1}^{2} P\right)+O\left(h^{2}\right)$.

Using the identification lemma B.2 below, this leads directly to the identifications:

$$
\begin{gathered}
K(r, u, E, j)+\partial_{r} B(r, u, E, j)\left(E \cos \left(\theta_{1}\right)\right)^{2}=A_{E \partial_{1} P} \\
L(r, u, E, j)=B_{E \partial_{1} P} \\
M(r, u, E, j)+\partial_{r} B(r, u, E, j) \frac{\cos \theta_{1}}{(E r)^{2}}+\partial_{r} D(r, u, E, j)\left(E \cos \left(\theta_{1}\right)\right)^{2}=C_{E \partial_{1} P}-\frac{1}{2} A_{\partial_{1}^{2} P} \\
N(r, u, E, j)=D_{E \partial_{1} P}-\frac{1}{2} B_{\partial_{1}^{2} P}
\end{gathered}
$$

where $\theta_{1}=\theta_{1}(r, E, j)$ denotes as before the solution in $[-\pi / 2, \pi / 2)$ of $\sin \theta_{1}=j / E r$. On the right-hand sides, notation such as $A_{E \partial_{1} P}, B_{E \partial_{1} P}$ etc. means "the functions $A, B$ etc. associated to $E \partial_{1} P$ by the formulas of Proposition A.3".

To justify these identifications we are using the following:

Lemma B.2. Let $A$ and $B$ be two smooth real-valued functions. Then the values of

$$
\begin{aligned}
& \frac{1}{2 \pi h} \int A(r, u, E, j) e^{\frac{i j(u-\theta)}{h}} e^{i \frac{E r \cos \left(\theta-\theta_{0}\right)}{h}} d \theta d j \\
& \quad+\frac{1}{2 \pi h} \int B(r, u, E, j) \cos \left(\theta-\theta_{0}\right) e^{\frac{i j(u-\theta)}{h}} e^{i \frac{E r \cos \left(\theta-\theta_{0}\right)}{h}} d \theta d j
\end{aligned}
$$

for all $r, u, \theta_{0}, E$ determine $A$ and $B$ uniquely.

Proof. Integrating (B.4) along $e^{i n \theta_{0}} d \theta_{0}\left(\theta_{0} \in[0,2 \pi], n\right.$ an arbitrary integer) yields the value

$$
\begin{aligned}
\int A(r, u, E, n h) e^{i n(u-\theta)} e^{i \frac{E r \cos \left(\theta-\theta_{0}\right)}{h}} d \theta & \\
& +\int B(r, u, E, n h) \cos \left(\theta-\theta_{0}\right) e^{i n(u-\theta)} e^{i \frac{E r \cos \left(\theta-\theta_{0}\right)}{h}} d \theta
\end{aligned}
$$

If we take $n=n(h)$ a family of even integers growing like $1 / h$, application of the method of stationary phase yields that this is (up to $O(h)$ )

$$
\begin{aligned}
2 e^{i n u}(2 \pi h)^{1 / 2}\left[\operatorname { s i n } ^ { 1 / 2 } \theta _ { 1 } A \left(r, u, E, h n(h) \cos \left(-n \theta_{1}+E r h^{-1} \cos \theta_{1}+\pi / 4\right)\right.\right. \\
\left.+i B(r, u, E, h n(h)) \sin \left(-n \theta_{1}+E r h^{-1} \cos \theta_{1}+\pi / 4\right)\right]
\end{aligned}
$$


where $\theta_{1}$ is the solution in $[-\pi / 2, \pi / 2)$ of $\sin \theta_{1}=\frac{h n(h)}{E r}$. If $A$ and $B$ are continuous and real-valued then (B.6) suffices to determine $A$ and $B$.

B.3. Formal calculation of $\left[\Delta, \mathcal{A}_{H}(P)\right]$. We want to use the previous identities to find the formal expression of $\left[\Delta, \mathcal{A}_{H}(P)\right]$. Remember that $\mathcal{A}_{H}(P)$ is the operator we want to use in all our proofs, because it comes naturally into a "tangential" part and a "radial" part of degree 1.

If we compare the formal calculations leading to the expressions of $\left[\Delta, \mathcal{A}_{E}(P)\right]$ and $\left[\Delta, \mathcal{A}_{H}(P)\right]$, we see that they are identical and thus $\left[-i h \Delta / 2, \mathcal{A}_{H}(P)\right]$ applied to $e_{\xi}$ at $(-r \sin u, r \cos u)$ has the form

$$
\begin{aligned}
& \frac{1}{2 \pi h} \int K(r, u, \sqrt{2 H}, j) e^{\frac{i j(u-\theta)}{h}} e^{i \frac{E r \cos \left(\theta-\theta_{0}\right)}{h}} d \theta d j \\
&+ \frac{1}{2 \pi h} \int L(r, u, \sqrt{2 H}, j) E \cos \left(\theta-\theta_{0}\right) e^{\frac{i j(u-\theta)}{h}} e^{i \frac{E r \cos \left(\theta-\theta_{0}\right)}{h}} d \theta d j \\
&+\frac{i h}{2 \pi h} \int M(r, u, \sqrt{2 H}, j) e^{\frac{i j(u-\theta)}{h}} e^{i \frac{E r \cos \left(\theta-\theta_{0}\right)}{h}} d \theta d j \\
&+ \frac{i h}{2 \pi h} \int N(r, u, \sqrt{2 H}, j) E \cos \left(\theta-\theta_{0}\right) e^{\frac{i j(u-\theta)}{h}} e^{i \frac{E r \cos \left(\theta-\theta_{0}\right)}{h}} d \theta d j \\
&+(2 \pi h)^{-1} \int_{u-\pi / 2}^{u+\pi / 2} \partial_{r} B(r, u, \sqrt{2 H}, j)\left[\left(E \cos \left(\theta_{1}\right)\right)^{2}+i h \frac{\cos \theta_{1}}{(E r)^{2}}\right] e^{\frac{i j\left(\theta-\theta_{0}\right)}{h}} e^{i \frac{E r \cos (\theta-u)}{h}} d \theta d j \\
&+(2 \pi h)^{-1} \int_{u-\pi / 2}^{u+\pi / 2} i h \partial_{r} D(r, u, \sqrt{2 H}, j)\left(E \cos \left(\theta_{1}\right)\right)^{2} e^{\frac{i j\left(\theta-\theta_{0}\right)}{h}} e^{i \frac{E r \cos (\theta-u)}{h}} d \theta d j
\end{aligned}
$$

Note that $\theta_{1}=\theta_{1}(r, E, j)$ and that the symbol in the last two lines still depends on $E$ (this is why we treat it separately). Everywhere else in the symbol, $E$ has been replaced by $\sqrt{2 H}$. Note also that $\left(E \cos \left(\theta_{1}\right)\right)^{2}=E^{2}-\frac{j^{2}}{r^{2}}$.

From this and from the identifications of Section B.2, we deduce the final formula

Proposition B.3. There exists a function $R(r, u, E, \sqrt{2 H}, j)$ such that

$$
\begin{aligned}
& \text { (B.7) }\left[-i h \Delta / 2, \mathcal{A}_{H}(P)\right]=\mathcal{A}_{H}\left(E \partial_{1} P\right)-\frac{i h}{2} \mathcal{A}_{H}\left(\partial_{1}^{2} P\right)+O\left(h^{2}\right) \\
& +\partial_{r} B\left(r, u, \sqrt{2 h D_{t}}, h D_{u}\right) \circ\left(-h^{2} \Delta-2 h D_{t}\right)+i h R\left(r, u, \sqrt{-h^{2} \Delta}, \sqrt{2 h D_{t}}, h D_{u}\right) \circ\left(-h^{2} \Delta-2 h D_{t}\right)
\end{aligned}
$$

Proof. Indeed, the identifications of Section B.2 yield

$$
\begin{aligned}
\text { (B.8) }\left[-i h \Delta / 2, \mathcal{A}_{H}(P)\right]=I_{\sqrt{2 H}}\left(E \partial_{1} P\right)+I I_{\sqrt{2 H}}\left(E \partial_{1} P\right) \\
+i h\left(I I I_{\sqrt{2 H}}\left(E \partial_{1} P\right)-1 / 2 I_{\sqrt{2 H}}\left(\partial_{1}^{2} P\right)\right) \\
+i h\left(I V_{\sqrt{2 H}}\left(E \partial_{1} P\right)-1 / 2 I I_{\sqrt{2 H}}\left(\partial_{1}^{2} P\right)\right) \\
+\partial_{r} B\left(r, u, \sqrt{2 h D_{t}}, h D_{u}\right) \circ\left(-h^{2} \Delta-2 h D_{t}\right)+i h R\left(r, u, \sqrt{-h^{2} \Delta}, \sqrt{2 h D_{t}}, h D_{u}\right) \circ\left(-h^{2} \Delta-2 h D_{t}\right)
\end{aligned}
$$

where the function $R$ is defined by the identity $R(r, u, E, \sqrt{2 H}, j)\left(E^{2}-2 H\right)$ is

$$
R(r, u, E, \sqrt{2 H}, j)\left(E^{2}-2 H\right)=\partial_{r} B(r, u, \sqrt{2 H}, j)\left[\frac{\cos \theta_{1}(r, E, j)}{(E r)^{2}}-\frac{\cos \theta_{1}(r, \sqrt{2 H}, j)}{2 H r^{2}}\right]
$$

We can apply a simple division lemma (actually the Taylor integral formula) to write

$$
\frac{\cos \theta_{1}(r, E, j)}{(E r)^{2}}-\frac{\cos \theta_{1}(r, \sqrt{2 H}, j)}{2 H r^{2}}=S(r, u, E, j, \sqrt{2 H})\left(E^{2}-2 H\right),
$$

and thus

$$
R(r, u, E, \sqrt{2 H}, j)=\partial_{r} B(r, u, \sqrt{2 H}, j) S(r, u, E, j, \sqrt{2 H})
$$




\section{Appendix C. Regularity of Boundary DATA AND CONSEQUEnCES}

We recall the following classical "hidden regularity" of the boundary data of solutions of (1.1):

Proposition C.1. For every $T>0$ there exists a constant $C>0$ such that, for every $u^{0} \in H_{0}^{1}(\mathbb{D})$ and every $f \in L^{1}\left(0, T ; H_{0}^{1}(\mathbb{D})\right)$, the solution $u \in C^{0}\left([0, T] ; H_{0}^{1}(\mathbb{D})\right)$ of

$$
\begin{gathered}
\frac{1}{i} \frac{\partial u}{\partial t}=\left(-\frac{1}{2} \Delta+V\right) u+f \quad, t \in \mathbb{R}, \quad z \in \mathbb{D}, \\
u\rceil_{\partial \mathbb{D}}=0 \\
u\rceil_{t=0}=u^{0}
\end{gathered}
$$

satisfies

$$
\left\|\partial_{n} u\right\|_{L^{2}((0, T) \times \partial \mathbb{D})} \leq C\left(\left\|\nabla u^{0}\right\|_{L^{2}(\mathbb{D})}+\|f\|_{L^{1}\left(0, T ; H^{1}(\mathbb{D})\right)}\right) .
$$

We refer to [Leb92, p. 284] or [GL93, Lemma 2.1] for a proof.

Remark C.2. Because we have

$$
\frac{d}{d t}\left\langle\left(-\frac{\Delta}{2}+V\right) U_{V}(t) u^{0}, U_{V}(t) u^{0}\right\rangle=\left\langle\partial_{t} V U_{V}(t) u^{0}, U_{V}(t) u^{0}\right\rangle
$$

we see that there exists $C$ (depending on $T,\|V\|_{\infty}$ and $\left\|\partial_{t} V\right\|_{\infty}$ such that

$$
\begin{aligned}
T\left\|\nabla u^{0}\right\|_{L^{2}(\mathbb{D})}^{2} & \leq C \int_{0}^{T}\left\|U_{V}(t) u^{0}\right\|_{L^{2}(\mathbb{D})}^{2} d t+\int_{0}^{T}\left\|\nabla U_{V}(t) u^{0}\right\|_{L^{2}(\mathbb{D})}^{2} d t \\
\int_{0}^{T}\left\|\nabla U_{V}(t) u^{0}\right\|_{L^{2}(\mathbb{D})}^{2} d t & \leq T\left\|\nabla u^{0}\right\|_{L^{2}(\mathbb{D})}^{2} d t+C \int_{0}^{T}\left\|U_{V}(t) u^{0}\right\|_{L^{2}(\mathbb{D})}^{2} d t
\end{aligned}
$$

hence

$$
T\left\|\nabla u^{0}\right\|_{L^{2}(\mathbb{D})}^{2}-C T\left\|u^{0}\right\|_{L^{2}(\mathbb{D})}^{2} \leq \int_{0}^{T}\left\|\nabla U_{V}(t) u^{0}\right\|_{L^{2}(\mathbb{D})}^{2} d t \leq T\left\|\nabla u^{0}\right\|_{L^{2}(\mathbb{D})}^{2} d t+C T\left\|u^{0}\right\|_{L^{2}(\mathbb{D})}^{2}
$$

Proposition C.1 has the following consequences, which are used everywhere in the paper.

Let $A$ be an operator. For $T>0$ let $\chi_{T}(t)$ be a smooth compactly supported function on $\mathbb{R}$ such that $\left|\chi_{T}(t)\right| \leq 1$, taking the value 0 outside $(0, T)$. Obviously, for every $u^{0} \in H_{0}^{1}(\mathbb{D})$, for every $s>0$, we can write

$$
\leq\|A\|_{L^{2}\left((0, T), H^{-s}\left(\mathbb{R}^{2}\right)\right) \longrightarrow L^{2}\left((0, T) \times \mathbb{R}^{2}\right)}\left\|U_{V}(t) u^{0}\right\|_{L^{2}((0, T) \times \mathbb{D})}\left\|\partial_{n} U_{V}(t) u^{0} \otimes \delta_{\partial \mathbb{D}}\right\|_{L^{2}\left((0, T), H^{-s}\left(\mathbb{R}^{2}\right)\right)} .
$$

For any $s>1 / 2$, the standard trace estimates (see for instance [CP82, Chapter 2, Section 4]) imply that

$$
\left\|\partial_{n} U_{V}(t) u^{0} \otimes \delta_{\partial \mathbb{D}}\right\|_{L^{2}\left((0, T), H^{-s}\left(\mathbb{R}^{2}\right)\right)} \leq\left\|\partial_{n}\left(U_{V}(t) u^{0}\right)\right\|_{L^{2}((0, T) \times \partial \mathbb{D})}
$$

and by Proposition C.1 this is bounded by $C\left\|\nabla u^{0}\right\|_{L^{2}(\mathbb{D})}$.

Let now be $\epsilon>0$ (in the context of this paper, think of $\epsilon$ as being $h$ or $R^{-1}$ ). In the previous inequality we take $A=\mathrm{Op}_{1}\left(a\left(z, \epsilon \xi, t, \epsilon^{2} H\right)\right)=a\left(z, \epsilon D_{z}, t, \epsilon^{2} D_{t}\right)$ where $a$ is smooth, compactly supported in all variables, and is such that $\chi_{T}(t) \equiv 1$ on the support of $a$. We note then that $\chi_{T} A=A$ and $A \chi_{T}=A+O\left(\epsilon^{-\infty}\right)$. Using the fact that

$$
\|A\|_{L^{2}\left((0, T), H^{-s}\left(\mathbb{R}^{2}\right)\right) \longrightarrow L^{2}\left((0, T) \times \mathbb{R}^{2}\right)} \leq C(T, a) \epsilon^{-s},
$$

we obtain

$$
\frac{\epsilon^{2}}{2}\left\langle\chi_{T} U_{V}(t) u^{0}, A\left(\partial_{n}\left(U_{V}(t) u^{0}\right) \otimes \delta_{\partial \mathbb{D}}\right)\right\rangle_{L^{2}\left(\mathbb{R}^{2} \times \mathbb{R}\right)} \leq C(T, a) \epsilon^{1-s}\left\|u^{0}\right\|_{L^{2}(\mathbb{D})}\left\|\epsilon \nabla u^{0}\right\|_{L^{2}(\mathbb{D})}
$$

If we extend $U_{V}(t) u^{0}$ to take the value 0 outside $\mathbb{D}$, we have already noted that

$$
\frac{\epsilon^{2}}{2} \partial_{n}\left(U_{V}(t) u^{0}\right) \otimes \delta_{\partial \mathbb{D}}=\epsilon^{2}\left(-\frac{\Delta}{2}+V+i \partial_{t}\right) U_{V}(t) u^{0}
$$


and thus equation (C.5) (always for $A=\mathrm{Op}_{1}\left(a\left(z, \epsilon \xi, t, \epsilon^{2} H\right)\right)$ may be rewritten as

$$
\begin{aligned}
\left\langle\chi_{T} U_{V}(t) u^{0}, A\left(-\frac{\epsilon^{2} \Delta}{2}+\epsilon^{2} V+i \epsilon^{2} \partial_{t}\right) U_{V}(t) u^{0}\right\rangle^{L^{2}\left(\mathbb{R}^{2} \times \mathbb{R}\right)} & \\
& \leq C(T, a) \epsilon^{1-s}\left\|u^{0}\right\|_{L^{2}(\mathbb{D})}\left\|\epsilon \nabla u^{0}\right\|_{L^{2}(\mathbb{D})}
\end{aligned}
$$

As a consequence

$$
\begin{aligned}
& \left\langle\chi_{T} U_{V}(t) u^{0}, \mathrm{Op}_{1}\left(a\left(z, \epsilon \xi, t, \epsilon^{2} H\right)\left(\frac{\epsilon^{2}|\xi|^{2}}{2}-\epsilon^{2} H\right)\right) U_{V}(t) u^{0}\right\rangle_{L^{2}\left(\mathbb{R}^{2} \times \mathbb{R}\right)} \\
& \quad \leq C(T, a) \epsilon^{1-s}\left\|u^{0}\right\|_{L^{2}(\mathbb{D})}\left\|\epsilon \nabla u^{0}\right\|_{L^{2}(\mathbb{D})}+\epsilon^{2}\left|\int \chi_{T}(t)\left\langle V U_{V}(t) u^{0}, U_{V}(t) u^{0}\right\rangle_{L^{2}(\mathbb{D})} d t\right| .
\end{aligned}
$$

Finally, if $a$ is supported away from $\{H=0\}$ this implies

$$
\begin{aligned}
\left\langle\chi_{T} U_{V}(t) u^{0}, \mathrm{Op}_{1}\left(a\left(z, \epsilon \xi, t, \epsilon^{2} H\right)\right.\right. & \left.\left.\left(\frac{|\xi|^{2}}{2 H}-1\right)\right) U_{V}(t) u^{0}\right\rangle_{L^{2}\left(\mathbb{R}^{2} \times \mathbb{R}\right)} \\
& \leq C(T, a) \epsilon^{1-s}\left\|u^{0}\right\|_{L^{2}(\mathbb{D})}\left(\left\|\epsilon \nabla u^{0}\right\|_{L^{2}(\mathbb{D})}+\left\|u^{0}\right\|_{L^{2}(\mathbb{D})}\right) .
\end{aligned}
$$

Since $a$ is compactly supported with respect to $H$, let us now show that we can actually replace $\left\|\epsilon \nabla u^{0}\right\|_{L^{2}(\mathbb{D})}$ by $\left\|u^{0}\right\|_{L^{2}(\mathbb{D})}$ in the last estimate (up to a constant depending on the support of $a$ ). The argument is easy if $V$ does not depend on time, but requires some care for time-dependent $V$. To see that, introduce a compactly supported function $g$ on $\mathbb{R}$ such that $a(z, \xi, t, H) g(H)=$ $a(z, \xi, t, H)$ (i.e. $g=1$ in a neighborhood of $\operatorname{supp}(a)$ ).

Let

$$
\left.w(t)=g\left(\epsilon^{2} D_{t}\right) U_{V}(t) u^{0}, \quad \text { and } \quad w^{0}=w\right\rceil_{t=0} .
$$

If $V$ does not depend on time, we have

$$
w=U_{V}(t) g\left(-\frac{\epsilon^{2} \Delta_{D}}{2}+\epsilon^{2} V\right) u^{0}, \quad \text { and } \quad w^{0}=g\left(-\frac{\epsilon^{2} \Delta_{D}}{2}+\epsilon^{2} V\right) u^{0}
$$

so we can replace $u^{0}$ by $\tilde{u}^{0}=g\left(-\frac{\epsilon^{2} \Delta}{2}+\epsilon^{2} V\right) u^{0}$ in the previous argument, and it is obvious that $\left\|\epsilon \nabla \tilde{u}^{0}\right\|_{L^{2}(\mathbb{D})} \leq C(g)\left\|u^{0}\right\|_{L^{2}(\mathbb{D})}$.

For general $V$, we write $\left[g\left(\epsilon^{2} D_{t}\right), V\right]=O\left(\epsilon^{2}\right)\left\|\partial_{t} V\right\|_{\infty}$, where the estimate of the remainder holds in the operator norm from $L_{\text {comp }}^{\infty}\left(\mathbb{R}_{t}, L^{2}(\mathbb{D})\right)$ to $L_{\text {loc }}^{\infty}\left(\mathbb{R}_{t}, L^{2}(\mathbb{D})\right)$. We have

$$
\frac{1}{i} \frac{\partial}{\partial t} w=\left(-\frac{\Delta}{2}+V\right) w+\left[g\left(\epsilon^{2} D_{t}\right), V\right] w .
$$

Thus

$$
\begin{aligned}
\left\langle\chi_{T}\right. & \left.U_{V}(t) u^{0}, \mathrm{Op}_{1}\left(a\left(z, \epsilon \xi, t, \epsilon^{2} H\right)\left(\frac{\epsilon^{2}|\xi|^{2}}{2}-\epsilon^{2} H\right)\right) U_{V}(t) u^{0}\right\rangle_{L^{2}\left(\mathbb{R}^{2} \times \mathbb{R}\right)} \\
= & \left\langle\chi_{T} U_{V}(t) u^{0}, \mathrm{Op}_{1}\left(a\left(z, \epsilon \xi, t, \epsilon^{2} H\right)\left(\frac{\epsilon^{2}|\xi|^{2}}{2}-\epsilon^{2} H\right)\right) w\right\rangle_{L^{2}\left(\mathbb{R}^{2} \times \mathbb{R}\right)} \\
= & \left\langle\chi_{T} U_{V}(t) u^{0}, \mathrm{Op}_{1}\left(a\left(z, \epsilon \xi, t, \epsilon^{2} H\right)\left(\frac{\epsilon^{2}|\xi|^{2}}{2}-\epsilon^{2} H\right)\right) U_{V}(t) w^{0}\right\rangle_{L^{2}\left(\mathbb{R}^{2} \times \mathbb{R}\right)} \\
& +O_{T}\left(\epsilon^{2}\right)\left\|\partial_{t} V\right\|_{\infty}\left\|u^{0}\right\|_{L^{2}(\mathbb{D})}^{2},
\end{aligned}
$$

where $w^{0}$ is defined in C.9. 
We know from the bilinear version of inequality (C.7) that

$$
\begin{aligned}
& \left\langle\chi_{T} U_{V}(t) u^{0}, \mathrm{Op}_{1}\left(a\left(x, \epsilon \xi, t, \epsilon^{2} H\right)\left(\frac{\epsilon^{2}|\xi|^{2}}{2}-\epsilon^{2} H\right)\right) U_{V}(t) w^{0}\right\rangle_{L^{2}\left(\mathbb{R}^{2} \times \mathbb{R}\right)} \\
& \leq C(T, a) \epsilon^{1-s}\left\|u^{0}\right\|_{L^{2}(\mathbb{D})}\left\|\epsilon \nabla w^{0}\right\|_{L^{2}(\mathbb{D})}+\epsilon^{2}\left|\int \chi_{T}(t)\left\langle V U_{V}(t) u^{0}, U_{V}(t) w^{0}\right\rangle_{L^{2}(\mathbb{D})} d t\right| \leq C(T, a) \epsilon^{1-s}\left\|u^{0}\right\|_{L^{2}(\mathbb{D})}\left\|\epsilon \nabla w^{0}\right\|_{L^{2}(\mathbb{D})}+\epsilon^{2} C T\left\|u^{0}\right\|_{L^{2}(\mathbb{D})}^{2} .
\end{aligned}
$$

Now we can use (C.2)-(C.3) in the form

$$
\begin{gathered}
T\left\|\nabla w^{0}\right\|_{L^{2}(\mathbb{D})}^{2} \leq C T\left(1+\epsilon^{2}\right)\left\|u^{0}\right\|_{L^{2}(\mathbb{D})}^{2}+\int_{0}^{T}\|\nabla w(t)\|_{L^{2}(\mathbb{D})}^{2} \\
\leq C T\left(1+\epsilon^{2}\right)\left\|u^{0}\right\|_{L^{2}(\mathbb{D})}^{2}+2 \int_{0}^{T}\left\langle\left(-\frac{\Delta}{2}+V\right) w(t), w(t)\right\rangle_{L^{2}(\mathbb{D})} d t \\
\leq C T\left(1+\epsilon^{2}\right)\left\|u^{0}\right\|_{L^{2}(\mathbb{D})}^{2}+2\left|\int_{0}^{T}\left\langle D_{t} w(t), w(t)\right\rangle_{L^{2}(\mathbb{D})} d t\right| \\
\leq C T\left(1+\epsilon^{2}\right)\left\|u^{0}\right\|_{L^{2}(\mathbb{D})}^{2}+2\left|\int_{0}^{T}\left\langle D_{t} g\left(\epsilon^{2} D_{t}\right) u(t), g\left(\epsilon^{2} D_{t}\right) u(t)\right\rangle_{L^{2}(\mathbb{D})} d t\right| \\
\leq C T\left(1+\epsilon^{2}\right)\left\|u^{0}\right\|_{L^{2}(\mathbb{D})}^{2}+C T \epsilon^{-2}\left\|u^{0}\right\|_{L^{2}(\mathbb{D})}^{2}
\end{gathered}
$$

This finally shows that the term $\left\|\epsilon \nabla w^{0}\right\|_{L^{2}(\mathbb{D})}$ on the right-hand side of (C.11) is bounded by $C T\left\|u^{0}\right\|_{L^{2}(\mathbb{D})}$. Finally, what we have shown is that, for $a(x, \xi, t, H)$ compactly supported, and supported away from $H=0$, we have

$$
\begin{aligned}
&\left\langle\chi_{T} U_{V}(t) u^{0}, \mathrm{Op}_{1}\left(a\left(z, \epsilon \xi, t, \epsilon^{2} H\right)\left(\frac{|\xi|^{2}}{2 H}-1\right)\right) U_{V}(t) u^{0}\right\rangle_{L^{2}\left(\mathbb{R}^{2} \times \mathbb{R}\right)} \\
& \leq C(T, a) \epsilon^{1-s}\left\|u^{0}\right\|_{L^{2}(\mathbb{D})}^{2}
\end{aligned}
$$

for all $u^{0} \in L^{2}(\mathbb{D})$ (that is to say, we do not need to assume a priori that $u^{0}$ is $\epsilon$-oscillating).

Similarly, if $a$ is supported away from $\xi=0$ and compactly supported, we have

$$
\begin{aligned}
&\left\langle\chi_{T} U_{V}(t) u^{0}, \mathrm{Op}_{1}\left(a\left(z, \epsilon \xi, t, \epsilon^{2} H\right)\left(\frac{2 H}{|\xi|^{2}}-1\right)\right) U_{V}(t) u^{0}\right\rangle_{L^{2}\left(\mathbb{R}^{2} \times \mathbb{R}\right)} \\
& \leq C(T, a) \epsilon^{1-s}\left\|u^{0}\right\|_{L^{2}(\mathbb{D})}^{2}
\end{aligned}
$$

Remark C.3. Let us summarize the properties of the function $\left.w^{0}=g\left(\epsilon^{2} D_{t}\right) U_{V}(t) u^{0}\right\rceil_{t=0}$.

- For fixed $\epsilon>0$, the operator $u^{0} \mapsto w^{0}$ is compact on $L^{2}(\mathbb{D})$ since we have proved $\left\|\epsilon \nabla w^{0}\right\|_{L^{2}(\mathbb{D})} \leq C\left\|u^{0}\right\|_{L^{2}(\mathbb{D})}$.

- By continuity of $t \mapsto U_{V}(t) u^{0}$, we have $w^{0} \underset{L^{2}(\mathbb{D})}{\longrightarrow} u^{0}$ as $\epsilon \longrightarrow 0$.

- For compactly supported a satisfying ga=a we have

$$
\begin{aligned}
\left\langle U_{V}(t) w^{0}, \mathrm{Op}_{1}(a(x, \epsilon \xi,\right. & \left.\left.\left.t, \epsilon^{2} H\right)\right) U_{V}(t) w^{0}\right\rangle_{L^{2}\left(\mathbb{R}^{2} \times \mathbb{R}\right)} \\
& =\left\langle U_{V}(t) u^{0}, \mathrm{Op}_{1}\left(a\left(x, \epsilon \xi, t, \epsilon^{2} H\right)\right) U_{V}(t) u^{0}\right\rangle_{L^{2}\left(\mathbb{R}^{2} \times \mathbb{R}\right)}+o(1)_{\epsilon}\left\|u^{0}\right\|_{L^{2}(\mathbb{D})}^{2}
\end{aligned}
$$

Finally, we prove by dyadic decomposition a statement similar to (C.12) and (C.13) for homogeneous functions:

Proposition C.4. Let $a(z, \xi, t, H)$ be a smooth function, compactly supported in $(z, t)$, and with the following homogeneity property : there exist $R_{0}>0$ such that

$$
a(z, \xi, t, H)=a\left(z, \lambda \xi, t, \lambda^{2} H\right), \quad \text { for }|\xi|^{2}+|H|>R_{0} \text { and } \lambda \geq 1 .
$$


Fix $s \in(1 / 2,1)$. If a vanishes in a neighbourhood of the set $\left\{|\xi|^{2}=2 H\right\}$ then for $R$ large enough

$$
\begin{aligned}
\left\langle U_{V}(t) u^{0}, \mathrm{Op}_{1}\left(a(z, \xi, t, H)\left(1-\chi\left(\frac{|\xi|^{2}+|H|}{R^{2}}\right)\right)\right) U_{V}(t) u^{0}\right. & \\
& \leq C(T, a) R^{s-1}\left\|u^{0}\right\|_{L^{2}(\mathbb{D})}^{2} \\
& \left.\leq C \mathbb{R}^{2} \times \mathbb{R}\right)
\end{aligned}
$$

Proof. To see that, decompose

$$
\begin{aligned}
a(z, \xi, t, H)\left(1-\chi\left(\frac{|\xi|^{2}+|H|}{R^{2}}\right)\right)= \\
\quad \sum_{k=0}^{\infty} a\left(z, 2^{-k} R^{-1} \xi, t, 2^{-2 k} R^{-2} H\right)\left(\chi\left(\frac{|\xi|^{2}+|H|}{2^{2(k+1)} R^{2}}\right)-\chi\left(\frac{|\xi|^{2}+|H|}{2^{2 k} R^{2}}\right)\right) .
\end{aligned}
$$

For each $k$ in the sum above, decompose further

$$
\begin{aligned}
\chi\left(\frac{|\xi|^{2}+|H|}{2^{2(k+1)} R^{2}}\right) & -\chi\left(\frac{|\xi|^{2}+|H|}{2^{2 k} R^{2}}\right) \\
= & \left(\chi\left(\frac{|\xi|^{2}+|H|}{2^{2(k+1)} R^{2}}\right)-\chi\left(\frac{|\xi|^{2}+|H|}{2^{2 k} R^{2}}\right)\right)\left(\chi\left(\frac{H}{2^{2 k-1} R^{2}}\right)+(1-\chi)\left(\frac{H}{2^{2 k-1} R^{2}}\right)\right)
\end{aligned}
$$

and note that we must have $|\xi|^{2} \geq 2^{2 k-1} R^{2}$ or $H \geq 2^{2 k-1} R^{2}$ on the support of this function.

If $a$ vanishes in a neighbourhood of the set $\left\{|\xi|^{2}=2 H\right\}$, we can write

$$
a(z, \xi, t, H)=b(z, \xi, t, H)\left(\frac{2 H}{|\xi|^{2}}-1\right)
$$

where $|\xi|^{2} \geq 2^{2 k-1} R^{2}$ and

$$
a(z, \xi, t, H)=b(z, \xi, t, H)\left(\frac{|\xi|^{2}}{2 H}-1\right)
$$

where $H \geq 2^{2 k-1} R^{2}$. Applying (C.12) and (C.13) for each $k$ (with $\epsilon=2^{-k} R^{-1}$ ), we finally obtain

$$
\begin{aligned}
\left\langle U_{V}(t) u^{0}, \mathrm{Op}_{1}\left(a(z, \xi, t, H)\left(1-\chi\left(\frac{|\xi|^{2}+|H|}{R^{2}}\right)\right)\right.\right. & ) U_{V}(t) u^{0}\right\rangle_{L^{2}\left(\mathbb{R}^{2} \times \mathbb{R}\right)} \\
& \leq C(T, a) \sum_{k=0}^{+\infty} R^{s-1} 2^{k(s-1)}\left\|u^{0}\right\|_{L^{2}(\mathbb{D})}^{2}
\end{aligned}
$$

which proves the proposition.

To conclude this section, we give a proof of Lemma 2.12.

Proof of Lemma 2.12. The operator $A\left(D_{t}\right) \varphi$ is bounded on $L^{2}(\mathbb{R} \times \mathbb{D})$, so

$$
\begin{aligned}
\left\|\nabla A\left(D_{t}\right) \varphi u\right\|_{L^{2}(\mathbb{R} \times \mathbb{D})}^{2}= & \left\langle-\Delta A\left(D_{t}\right) \varphi u, A\left(D_{t}\right) \varphi u\right\rangle_{L^{2}(\mathbb{R} \times \mathbb{D})} \\
= & \left\langle A\left(D_{t}\right) \varphi(-\Delta) u, A\left(D_{t}\right) \varphi u\right\rangle_{L^{2}(\mathbb{R} \times \mathbb{D})} \\
& +\left\langle\left[-\Delta, A\left(D_{t}\right) \varphi\right] u, A\left(D_{t}\right) \varphi u\right\rangle_{L^{2}(\mathbb{R} \times \mathbb{D})} .
\end{aligned}
$$

One the one hand, we have

$$
\begin{aligned}
\left|\left\langle\left[-\Delta, A\left(D_{t}\right) \varphi\right] u, A\left(D_{t}\right) \varphi u\right\rangle_{L^{2}(\mathbb{R} \times \mathbb{D})}\right| & =\left|-\left\langle 2 \nabla \varphi \cdot \nabla u+u \Delta \varphi, A\left(D_{t}\right)^{2} \varphi u\right\rangle_{L^{2}(\mathbb{R} \times \mathbb{D})}\right| \\
& \leq 2\left|\left\langle u, \operatorname{div}\left\{\nabla \varphi\left(A\left(D_{t}\right)^{2} \varphi u\right)\right\}\right\rangle_{L^{2}(\mathbb{R} \times \mathbb{D})}\right|+C\|\tilde{\varphi} u\|_{L^{2}(\mathbb{R} \times \mathbb{D})}^{2} \\
& \leq 2\left|\left\langle u, \nabla \varphi \cdot \nabla\left(A\left(D_{t}\right)^{2} \varphi u\right)\right\rangle_{L^{2}(\mathbb{R} \times \mathbb{D})}\right|+C\|\tilde{\varphi} u\|_{L^{2}(\mathbb{R} \times \mathbb{D})}^{2} \\
& \leq \varepsilon\left\|\nabla A\left(D_{t}\right) \varphi u\right\|_{L^{2}(\mathbb{R} \times \mathbb{D})}^{2}+C\left(1+\varepsilon^{-1}\right)\|\tilde{\varphi} u\|_{L^{2}(\mathbb{R} \times \mathbb{D})}^{2}
\end{aligned}
$$

for some $\tilde{\varphi}$ equal to one on the support of $\varphi$, for all $\varepsilon>0$. 
On the other hand, since $u$ solves (1.1), we have

$$
\begin{aligned}
\left|\left\langle A\left(D_{t}\right) \varphi(-\Delta) u, A\left(D_{t}\right) \varphi u\right\rangle_{L^{2}(\mathbb{R} \times \mathbb{D})}\right| & =\left|\left\langle A\left(D_{t}\right) \varphi\left(2 D_{t}-V\right) u, A\left(D_{t}\right) \varphi u\right\rangle_{L^{2}(\mathbb{R} \times \mathbb{D})}\right| \\
& \leq\left|\left\langle A\left(D_{t}\right) \varphi\left(2 D_{t}-V\right) u, A\left(D_{t}\right) \varphi u\right\rangle_{L^{2}(\mathbb{R} \times \mathbb{D})}\right| \\
& \leq 2\left|\left\langle A\left(D_{t}\right)^{2} \varphi D_{t} u, \varphi u\right\rangle_{L^{2}(\mathbb{R} \times \mathbb{D})}\right|+C\|\tilde{\varphi} u\|_{L^{2}(\mathbb{R} \times \mathbb{D})}^{2} \\
& \leq 2\left|\left\langle A\left(D_{t}\right)^{2} D_{t} \varphi u, \varphi u\right\rangle_{L^{2}(\mathbb{R} \times \mathbb{D})}\right|+C\|\tilde{\varphi} u\|_{L^{2}(\mathbb{R} \times \mathbb{D})}^{2} \\
& \leq C\|\tilde{\varphi} u\|_{L^{2}(\mathbb{R} \times \mathbb{D})}^{2},
\end{aligned}
$$

since $A\left(D_{t}\right)^{2} D_{t}=1 / 2 \mathrm{Op}_{1}\left(\psi^{2}(H)\right)$ is bounded. Collecting these estimates in (C.16), recalling that $\|\tilde{\varphi} u\|_{L^{2}(\mathbb{R} \times \mathbb{D})}^{2} \leq C\left\|u^{0}\right\|_{L^{2}(\mathbb{D})}^{2}$, and taking $\varepsilon$ sufficiently small concludes the proof of Lemma 2.12.

\section{Appendix D. Time Regularity of Wigner measures}

In this section we present a proof of the following (general) result on time regularity of semiclassical measures associated to solutions of the Schrödinger equation (1.1). Even if not stated here, its microlocal counterpart also holds.

Proposition D.1. Let $\mu_{s c}$ be obtained as a limit (2.4). Then there exists $\mu \in L^{\infty}\left(\mathbb{R}_{t} ; \mathcal{M}_{+}\left(T^{*} \mathbb{R}^{2}\right)\right)$ such that, for every $a \in C_{c}^{\infty}\left(T^{*} \mathbb{R}^{2} \times T^{*} \mathbb{R}\right)$ we have:

$$
\int_{T^{*} \mathbb{R}^{2} \times T^{*} \mathbb{R}} a(z, \xi, t, H) \mu_{s c}(d z, d \xi, d t, d H)=\int_{\mathbb{R}} \int_{T^{*} \mathbb{R}^{2}} a\left(z, \xi, t, \frac{|\xi|^{2}}{2}\right) \mu(t, d z, d \xi) d t .
$$

Proof. Let $u_{h}(\cdot, t):=U_{V}(t) u_{h}^{0}$ and note that the Wigner distributions:

$$
\tilde{W}_{u_{h}}^{h}(t): C_{c}^{\infty}\left(T^{*} \mathbb{R}^{2}\right) \ni l \longmapsto\left\langle U_{V}(t) u_{h}^{0}, \mathrm{Op}_{h}(l) U_{V}(t) u_{h}^{0}\right\rangle_{L^{2}\left(\mathbb{R}^{2}\right)} \in \mathbb{C}
$$

are uniformly bounded in $L^{\infty}\left(\mathbb{R}_{t} ; \mathcal{D}^{\prime}\left(T^{*} \mathbb{R}^{2}\right)\right)$. Hence, possibly after extracting a subsequence, we can assume that, for every $b \in C_{c}^{\infty}\left(T^{*} \mathbb{R}^{2} \times \mathbb{R}\right)$ :

$$
\lim _{h \rightarrow 0^{+}} \int_{\mathbb{R}}\left\langle U_{V}(t) u_{h}^{0}, \mathrm{Op}_{h}(b(\cdot, t)) U_{V}(t) u_{h}^{0}\right\rangle_{L^{2}\left(\mathbb{R}^{2}\right)}=\int_{\mathbb{R}} \int_{T^{*} \mathbb{R}^{2}} b(z, \xi, t) \tilde{\mu}_{s c}(t, d z, d \xi) d t
$$

and (using the sharp Gårding inequality) the limiting Wigner distribution is a nonnegative measure $\tilde{\mu}_{s c} \in L^{\infty}\left(\mathbb{R}_{t} ; \mathcal{M}_{+}\left(T^{*} \mathbb{R}^{2}\right)\right)$. We next show that for any $b \in C_{c}^{\infty}\left(T^{*} \mathbb{R}^{2} \times \mathbb{R}\right)$ with $b \geq 0$ one has:

$$
\int_{T^{*} \mathbb{R}^{2} \times T^{*} \mathbb{R}} b(z, \xi, t) \mu_{s c}(d z, d \xi, d t, d H) \leq \int_{\mathbb{R}} \int_{T^{*} \mathbb{R}^{2}} b(z, \xi, t) \tilde{\mu}_{s c}(t, d z, d \xi) d t .
$$

To see this, let $\chi \in C_{c}^{\infty}(\mathbb{R})$ be a cut-off function satisfying $0 \leq \chi \leq 1$, strictly positive in $(-3 / 2,3 / 2)$, vanishing outside that interval, and such that $\chi\rceil_{(-1,1)} \equiv 1$. Write, for $R>0$, $\chi_{R}:=\chi(\cdot / R)$ and $\sigma_{R}:=\sqrt{1-\chi_{R}}$. Then we have:

$$
\left\langle u_{h}, \mathrm{Op}_{h}(b) \chi_{R}\left(h^{2} D_{t}\right) u_{h}\right\rangle_{L^{2}\left(\mathbb{R}^{2} \times \mathbb{R}\right)}=\left\langle u_{h}, \mathrm{Op}_{h}(b) u_{h}\right\rangle_{L^{2}\left(\mathbb{R}^{2} \times \mathbb{R}\right)}+k_{h, R}(b)+O(h),
$$

where:

$$
k_{h, R}(b):=\left\langle\sigma_{R}\left(h^{2} D_{t}\right) u_{h}, \mathrm{Op}_{h}(b) \sigma_{R}\left(h^{2} D_{t}\right) u_{h}\right\rangle_{L^{2}\left(\mathbb{R}^{2} \times \mathbb{R}\right)} .
$$

Taking limits in (D.2) as $h \rightarrow 0^{+}$we find that:

$$
\begin{aligned}
& \int_{T^{*} \mathbb{R}^{2} \times T^{*} \mathbb{R}} b(z, \xi, t) \chi_{R}(H) \mu_{s c}(d z, d \xi, d t, d H) \\
&=\int_{\mathbb{R}} \int_{T^{*} \mathbb{R}^{2}} b(z, \xi, t) \tilde{\mu}_{s c}(t, d z, d \xi) d t+\lim _{h \rightarrow 0^{+}} k_{h, R}(b) .
\end{aligned}
$$

But clearly, as $b \geq 0$, we always have

$$
\lim _{h \rightarrow 0^{+}} k_{h, R}(b)=\lim _{h \rightarrow 0^{+}} \int_{\mathbb{R}} \tilde{W}_{\sigma_{R}\left(h^{2} D_{t}\right) u_{h}}^{h}(b(t, \cdot)) d t \geq 0,
$$

for every $R>0$. Taking this into account and letting $R \rightarrow \infty$ in (D.3) proves (D.1). 
Now, as a consequence of (D.1) we have that the image of $\mu_{s c}$ under the projection onto the $H$ component is of the form $\mu(t, \cdot) d t$ for some $\mu \in L^{\infty}\left(\mathbb{R}_{t} ; \mathcal{M}_{+}\left(T^{*} \mathbb{R}^{2}\right)\right)$. The disintegration theorem then ensures that $\mu_{s c}$ can be written as:

$$
\mu_{s c}(d z, d \xi, d t, d H)=\mu_{x, \xi, t}(d H) \mu(t, d z, d \xi) d t
$$

Since $\mu_{s c}$ is supported on the characteristic set $|\xi|^{2}=2 H$ we conclude that $\mu_{x, \xi, t}(d H)=\delta_{|\xi|^{2} / 2}(d H)$ and the result follows.

\section{REFERENCES}

[AFKM14] Nalini Anantharaman, Clotilde Fermanian-Kammerer, and Fabricio Macià. Semiclassical completely integrable systems : Long-time dynamics and observability via two-microlocal wigner measures. to appear in Amer. J. Math., 2014.

[AL14] Nalini Anantharaman and Matthieu Léautaud. Sharp polynomial decay rates for the damped wave equation on the torus. Anal. PDE, 7(1):159-214, 2014.

[ALM15] Nalini Anantharaman, Matthieu Léautaud, and Fabricio Macià. Delocalization of quasimodes on the disk. In preparation, 2015.

[AM10] Daniel Azagra and Fabricio Macià. Concentration of symmetric eigenfunctions. Nonlinear Anal., 73(3):683-688, 2010.

[AM12] Nalini Anantharaman and Fabricio Macià. The dynamics of the Schrödinger flow from the point of view of semiclassical measures. In Spectral geometry, volume 84 of Proc. Sympos. Pure Math., pages 93-116. Amer. Math. Soc., Providence, RI, 2012.

[AM14] Nalini Anantharaman and Fabricio Macià. Semiclassical measures for the Schrödinger equation on the torus. to appear in J. Eur. Math. Soc., 2014.

[AMJ12] Tayeb Aissiou, Fabricio Macià, and Dmitry Jakobson. Uniform estimates for the solutions of the schrödinger equation on the torus and regularity of semiclassical measures. Mathematical Research Letters, 19(3):589-599, 2012.

[Ana08] Nalini Anantharaman. Entropy and the localization of eigenfunctions. Ann. of Math. (2), 168(2):435$475,2008$.

[AR12] Nalini Anantharaman and Gabriel Rivière. Dispersion and controllability for the Schrödinger equation on negatively curved manifolds. Anal. PDE, 5(2):313-338, 2012.

[BBZ13] Jean Bourgain, Nicolas Burq, and Maciej Zworski. Control for Schrödinger operators on 2-tori: rough potentials. J. Eur. Math. Soc. (JEMS), 15(5):1597-1628, 2013.

[BLR92] Claude Bardos, Gilles Lebeau, and Jeffrey Rauch. Sharp sufficient conditions for the observation, control, and stabilization of waves from the boundary. SIAM J. Control Optim., 30(5):1024-1065, 1992.

[Bou97] Jean Bourgain. Analysis results and problems related to lattice points on surfaces. In Harmonic analysis and nonlinear differential equations (Riverside, CA, 1995), volume 208 of Contemp. Math., pages 85109. Amer. Math. Soc., Providence, RI, 1997.

[BR02] A. Bouzouina and D. Robert. Uniform semiclassical estimates for the propagation of quantum observables. Duke Math. J., 111(2):223-252, 2002.

[Bur13] Nicolas Burq. Semiclassical measures for inhomogeneous Schrödinger equations on tori. Anal. PDE, 6(6):1421-1427, 2013.

[BZ04] Nicolas Burq and Maciej Zworski. Control theory and high energy eigenfunctions. In Journées "Équations aux Dérivées Partielles", pages Exp. No. XIII, 10. École Polytech., Palaiseau, 2004.

[BZ12] Nicolas Burq and Maciej Zworski. Control for Schrödinger operators on tori. Math. Res. Lett., 19(2):309-324, 2012.

[CP82] Jacques Chazarain and Alain Piriou. Introduction to the theory of linear partial differential equations, volume 14 of Studies in Mathematics and its Applications. North-Holland Publishing Co., Amsterdam, 1982. Translated from the French.

[CV71] Alberto-P. Calderón and Rémi Vaillancourt. On the boundedness of pseudo-differential operators. J. Math. Soc. Japan, 23:374-378, 1971.

[FK00a] Clotilde Fermanian-Kammerer. Mesures semi-classiques 2-microlocales. C. R. Acad. Sci. Paris Sér. I Math., 331(7):515-518, 2000.

[FK00b] Clotilde Fermanian Kammerer. Propagation and absorption of concentration effects near shock hypersurfaces for the heat equation. Asymptot. Anal., 24(2):107-141, 2000.

[FKG02] Clotilde Fermanian-Kammerer and Patrick Gérard. Mesures semi-classiques et croisement de modes. Bull. Soc. Math. France, 130(1):123-168, 2002.

[Gér91a] Patrick Gérard. Mesures semi-classiques et ondes de Bloch. In Séminaire sur les Équations aux Dérivées Partielles, 1990-1991, pages Exp. No. XVI, 19. École Polytech., Palaiseau, 1991.

[Gér91b] Patrick Gérard. Microlocal defect measures. Comm. Partial Differential Equations, 16(11):1761-1794, 1991. 
[Gér98] Patrick Gérard. Description du défaut de compacité de l'injection de Sobolev. ESAIM Control Optim. Calc. Var., 3:213-233 (electronic), 1998.

[GL93] Patrick Gérard and Éric Leichtnam. Ergodic properties of eigenfunctions for the Dirichlet problem. Duke Math. J., 71(2):559-607, 1993.

[GMMP97] Patrick Gérard, Peter A. Markowich, Norbert J. Mauser, and Frédéric Poupaud. Homogenization limits and Wigner transforms. Comm. Pure Appl. Math., 50(4):323-379, 1997.

[Har89] Alain Haraux. Séries lacunaires et contrôle semi-interne des vibrations d'une plaque rectangulaire. $J$. Math. Pures Appl. (9), 68(4):457-465 (1990), 1989.

[Hör76] Lars Hörmander. Linear partial differential operators. Springer Verlag, Berlin, 1976.

[Jaf90] Stéphane Jaffard. Contrôle interne exact des vibrations d'une plaque rectangulaire. Portugal. Math., 47(4):423-429, 1990.

[Jak97] Dmitry Jakobson. Quantum limits on flat tori. Ann. of Math. (2), 145(2):235-266, 1997.

[JZ99] Dmitry Jakobson and Steve Zelditch. Classical limits of eigenfunctions for some completely integrable systems. In Emerging applications of number theory (Minneapolis, MN, 1996), volume 109 of IMA Vol. Math. Appl., pages 329-354. Springer, New York, 1999.

[Kom92] Vilmos Komornik. On the exact internal controllability of a Petrowsky system. J. Math. Pures Appl. (9), 71(4):331-342, 1992.

[Lag83] John Lagnese. Control of wave processes with distributed controls supported on a subregion. SIAM J. Control Optim., 21(1):68-85, 1983.

[Lau10] Camille Laurent. Global controllability and stabilization for the nonlinear Schrödinger equation on some compact manifolds of dimension 3. SIAM J. Math. Anal., 42(2):785-832, 2010.

[Lau14] Camille Laurent. Internal control of the Schrödinger equation. Math. Control Rel. Fields, 4(2):161-186, 2014.

[Leb92] Gilles Lebeau. Contrôle de l'équation de Schrödinger. J. Math. Pures Appl. (9), 71(3):267-291, 1992.

[Leb96] Gilles Lebeau. Équation des ondes amorties. In Algebraic and geometric methods in mathematical physics (Kaciveli, 1993), volume 19 of Math. Phys. Stud., pages 73-109. Kluwer Acad. Publ., Dordrecht, 1996.

[Lio88] Jacques-Louis Lions. Exact controllability, stabilization and perturbations for distributed systems. SIAM Rev., 30(1):1-68, 1988.

[LP93] Pierre-Louis Lions and Thierry Paul. Sur les mesures de Wigner. Rev. Mat. Iberoamericana, 9(3):553618, 1993.

[LR97] Gilles Lebeau and Luc Robbiano. Stabilisation de l'équation des ondes par le bord. Duke Math. J., 86(3):465-491, 1997.

[Mac08] Fabricio Macià. Some remarks on quantum limits on Zoll manifolds. Comm. Partial Differential Equations, 33(4-6):1137-1146, 2008.

[Mac09] Fabricio Macià. Semiclassical measures and the Schrödinger flow on Riemannian manifolds. Nonlinearity, 22(5):1003-1020, 2009.

[Mac10] Fabricio Macià. High-frequency propagation for the Schrödinger equation on the torus. J. Funct. Anal., 258(3):933-955, 2010.

[Mac11] Fabricio Macià. The Schrödinger flow in a compact manifold: high-frequency dynamics and dispersion. In Modern aspects of the theory of partial differential equations, volume 216 of Oper. Theory Adv. Appl., pages 275-289. Birkhäuser/Springer Basel AG, Basel, 2011.

[Mil96] Luc Miller. Propagation d'ondes semi-classiques à travers une interface et mesures 2-microlocales. PhD thesis, École Polythecnique, Palaiseau, 1996.

[Mil97] Luc Miller. Short waves through thin interfaces and 2-microlocal measures. In Journées "Équations aux Dérivées Partielles" (Saint-Jean-de-Monts, 1997), pages Exp. No. XII, 12. École Polytech., Palaiseau, 1997.

[Nie96] Francis Nier. A semi-classical picture of quantum scattering. Ann. Sci. École Norm. Sup. (4), 29(2):149183, 1996.

[PTZ14] Yannick Privat, Emmanuel Trélat, and Enrique Zuazua. Optimal observability of the multi-dimensional wave and Schrödinger equations in quantum ergodic domains. J. Europ. Math. Soc., to appear, 2014.

[RTTT05] Karim Ramdani, Takéo Takahashi, Gérald Tenenbaum, and Marius Tucsnak. A spectral approach for the exact observability of infinite-dimensional systems with skew-adjoint generator. J. Funct. Anal., 226(1):193-229, 2005.

[RZ09] Luc Robbiano and Claude Zuily. The Kato smoothing effect for Schrödinger equations with unbounded potentials in exterior domains. Int. Math. Res. Not. IMRN, (9):1636-1698, 2009.

[Sie29] Carl Ludwig Siegel. Über einige Anwendungen diophantischer Approximationen. Abh. Preuß. Akad. Wiss., Phys.-Math. Kl., 1929(1):70 s., 1929.

[Wig32] Eugene P. Wigner. On the quantum correction for thermodynamic equilibrium. Phys. Rev., 40:749-759, 1932.

[Zwo12] Maciej Zworski. Semiclassical analysis, volume 138 of Graduate Studies in Mathematics. American Mathematical Society, Providence, RI, 2012. 
Université Paris-Sud 11, Mathématiques, BÂt. 425, 91405 Orsay Cedex, France

E-mail address: Nalini.Anantharaman@math.u-psud.fr

Université Paris Diderot, Institut de Mathématiques de Jussieu-Paris Rive Gauche, UmR 7586 , Bâtiment Sophie Germain, 75205 Paris Cedex 13 France

E-mail address: leautaud@math.univ-paris-diderot.fr

Universidad Politécnica de Madrid. DCAin, etsi Navales. Avda. Arco de la Victoria s/n. 28040 MADRID, SPAIN

E-mail address: Fabricio.Macia@upm.es 